\title{
Hepatocyte transplantation
}

\author{
Citation for published version (APA):
}

Vroemen, J. P. A. M. (1987). Hepatocyte transplantation. [Doctoral Thesis, Maastricht University]. Rijksuniversiteit Limburg. https://doi.org/10.26481/dis.19870618jv

Document status and date:

Published: 01/01/1987

DOI:

10.26481/dis.19870618jv

Document Version:

Publisher's PDF, also known as Version of record

\section{Please check the document version of this publication:}

- A submitted manuscript is the version of the article upon submission and before peer-review. There can be important differences between the submitted version and the official published version of record.

People interested in the research are advised to contact the author for the final version of the publication, or visit the DOI to the publisher's website.

- The final author version and the galley proof are versions of the publication after peer review.

- The final published version features the final layout of the paper including the volume, issue and page numbers.

Link to publication

\footnotetext{
General rights rights.

- You may freely distribute the URL identifying the publication in the public portal. please follow below link for the End User Agreement:

www.umlib.nl/taverne-license

Take down policy

If you believe that this document breaches copyright please contact us at:

repository@maastrichtuniversity.nl

providing details and we will investigate your claim.
}

Copyright and moral rights for the publications made accessible in the public portal are retained by the authors and/or other copyright owners and it is a condition of accessing publications that users recognise and abide by the legal requirements associated with these

- Users may download and print one copy of any publication from the public portal for the purpose of private study or research.

- You may not further distribute the material or use it for any profit-making activity or commercial gain

If the publication is distributed under the terms of Article $25 \mathrm{fa}$ of the Dutch Copyright Act, indicated by the "Taverne" license above, 
Hepatocyte transplantation 



\title{
Hepatocyte transplantation
}

\author{
Proefschrift
}

ter verkrijging van de graad van doctor in de geneeskunde aan de Rijksuniversiteit Limburg te Maastricht, op gezag van de Rector Magnificus, Prof. Dr. F.I.M. Bonke, volgens het besluit van het College van Dekanen, in het openbaar te verdedigen op donderdag 18 juni 1987 om vier uur

door

Joseph Pieter Anna Maria Vroemen

geboren te Geleen. 
Promotor: $\quad$ Prof. Dr. G. Kootstra

Co-promotores: Dr. W.A. Buurman

Dr. C.J. van der Linden

Referenten: Prof. Dr. J.A. Flendrig

Prof. Dr. K.P.M. Heirwegh

Prof. Dr. D.K.F. Meyer

Prof. Dr. med. R. Pichlmayr

The author wishes to thank the Jan Dekkerstichting \& Dr. Ludgardine Bouwmanstichting for financial support. 
Les utopies ne sont souvent que des vérités prématurées.

Lamartine 
General introduction

Survey of the literature

Introduction to the experimental work

References to the Preface and Chapter 1

CHAPTER 2

Treatment of enzyme deficiency by hepatocyte transplantation in rats (Journal of Surgical Research 1985; 39: 267)

CHAPTER 3

The congenic normal R/APfd and jaundiced $R / A P f d-j / j$ rat strains:

a new animal model of hereditary nonhemolytic unconjugated hyperbilirubinemia due to defective bilirubin conjugation

(Laboratory Animals 1986; 20: 335)

CHAPTER 4

Hepatocyte transplantation for enzyme deficiency disease in congenic rats (Transplantation 1986; 42: 130)

CHAPTER 5

Transplantation of isolated hepatocytes into the pancreas

(Accepted for publication in European Surgical Research)

CHAPTER 6

The cytokinetic behaviour of donor hepatocytes after syngenic hepatocyte transplantation into the spleen

(Accepted for publication in Transplantation) 
In vivo dynamic $\$$ m Tc-HIDA scintigraphy after hepatocyte transplantation: a new method for monitoring of graft function (European Surgical Research 1987; 19: 140)

CHAPTER 8

Summary and conclusions

Résumé et conclusions

Samenvatting en conclusies

Acknowledgements

Curriculum vitae 


\section{PREFACE}

The present thesis contains an experimental study of the transplantation of isolated hepatocytes. This transplantation technique has been proposed as a potential therapy for hereditary enzyme deficiency diseases. Preliminary results of hepatocyte transplantation were promising and offered perspectives for future clinical application.

Although in many enzyme deficiency diseases the specific enzymatic defects have been identified, most inborn errors of metabolism still constitute difficult management problems (1-4). It is evident, that gene therapy would be the causal, and thus the ideal solution to this problem. Progress is being made in DNA technology, but, as yet, gene therapy for hereditary metabolic disease still awaits clinical application (4-7). Results of current therapeutic strategies such as avoidance therapy (nutritional restriction measures as in phenylketonuria and galactosemia), drug therapy (for example with chelating agents in Wilson"s disease) and gene product replacement (e.g. administration of the missing enzyme or protein as in hemophilia) are often unsatisfactory, or hard to realize, costly and unpractical (2-4, 7-11). As a consequence, efforts directed towards alternative therapeutic methods merit profound study. With the discovery that a liver allograft provided donor specific protein phenotypes to the recipient, transplantation medicine opened new horizons to the treatment of inborns errors of metabolism (12-14). In liver transplant recipients, previously suffering from cirrhosis due to inborn errors of metabolism (which included alpha-1-antitrypsine deficiency, glycogen storage disease, tyrosinemia, galactose- 
mia, hemochromatosis, the Crigler-Najjar Syndrome and Wilson's disease), liver grafting was primarily performed as treatment for cirrhosis due to or associated with the enzymatic defect, not for the deficiency proper (15-24). Nevertheless, orthotopic liver transplantation proved to be capable of correcting these previously often fatal metabolic disorders leading to cirrhosis. In spite of the results obtained, there are only anecdotal examples of liver replacement for the sole purpose of treating enzyme deficiency disease; one successful case of simultaneous heart and liver transplantation for heart failure due to homozygous familial hypercholesterolemia, and one case of liver replacement for oxalosis (24). These achievements are encouraging indeed, but the complexity of procurement, the difficuit management of both donor and recipient and the high risk surgical procedure render liver transplantation a formidable enterprise associated with considerable morbidity and mortality. Therefore, whole (and also auxilliary) liver transplantation can not be considered at present as an established mode of therapy for hepatic based enzyme deficiency disease, unless it has caused or is associated with end stage hepatic failure.

Notwithstanding this, the concept of enzyme replacement by transplantation remains very attractive. For practical reasons, a far more simple and safe procedure other than replacing a whole organ would be desirable. Such a procedure would consist of transplanting tissue fragments or isolated cells as free grafts. Transplantation of tissue producing the missing enzyme could provide a treatment modality for enzyme deficiency disease at a level to be situated between enzyme replacement therapy and gene therapy. Two basic mechanisms of enzyme replacement by means of transplantation can be postulated: first, in case the enzymatic defect leads to toxic accumulation of substrate, grafted tissue can metabolize the accumulated substrate in situ, and thus have a detoxifying function. The second possibility is that grafted tissue releases the missing enzyme(s) or protein(s) into the circulation, which results in the therapeutic effect.

This new approach towards the treatment of hepatic based enzyme deficiency disease has been evaluated in the Gunn rat, an animal model of hepatic based enzyme deficiency disease. This rat strain suffers from an absolute deficiency of bilirubin uridyldiphosphate glucuronyl transferase (BGT) and consequently exhibits a lifelong unconjugated hyperbilirubinemia (25). In the human, this enzyme deficiency disease is known as the Crigler-Najjar Syndrome. Rugstad et al. (26) reported in 1970 that subcutaneous implantation of rat hepatoma cells resulted in the appearance of the deficient enzyme activity in the recipient Gunn rat. Mukherjee (27) showed in 1973 that intrahepatic grafting of liver fragments from normal donor rats into Gunn rats caused a reduction of the plasma bilirubin levels in the recipient animals. A few years later, Matas (28) and Groth (29) demonstrated, independently, that transplantation of isolated hepatocytes brought about a similar beneficial effect. The possibility of correcting a specific enzymatic defect by a hepatocellular transplant suggested that this transplantation technique could also provide lifesustaining metabolic support in other states of hepatic insufficiecy, in particular acute liver failure. This resulted in various reports that described an increased sur- 
vival in experimentally induced acute liver failure in animals after hepatocyte tranplantation (30-32).

In 1982, research activities into hepatocyte transplantation were started in our institution. The aim of the present study was:

1. to explore the potential of hepatocyte transplantation in the treatment of enzyme deficiency disease and to investigate the mechanism(s) by which this transplantation procedure was effective.

2. to study the behaviour of the hepatocellular graft, with special emphasis on biological and cytokinetic aspects.

The results of the endeavours to realize these objectives are condensed in the publications underlying this thesis. 



\section{CHAPTER 1}

\section{Generall introduction}

\section{Survey of the literature}

\section{Hepatocyte transplantation (HTX) for enzyme deficiency disease.}

The first report describing the transfer of specific enzyme activity to an enzymedeficient animal by tissue transplantation was published by Rugstad (26) in 1970 . Cells from a clonal strain of rat hepatoma were transplanted subcutaneously into two Gunn rats. In this rat strain bilirubin uridyldiphosphate glucuronyl transferase (BGT) is totally absent from the liver, causing a life-long unconjugated hyperbilirubinemia $(25,33)$. After transplantation, the recipient rats appeared to be capable of conjugating bilirubin, and serum bilirubin levels declined significantly. Mukherjee (27) stated that transplantation of neoplastic tissue held little promise for future clinical application. As an alternative to Rugstad's approach, he grafted small liver fragments of normal Wistar liver taken by punch biopsy into the liver of Gunn rats (27). He also found a reduction of total plasma bilirubin in the recipient rats. In liver homogenates of the treated Gunn rats BGT activity was detected, in contrast to liver homogenates of untreated Gunn rats.

By then, it was generally known that isolated hepatocytes maintained, at least temporarily, their metabolic capacities in vitro (34-36). Matas (28) and Sutherland (37) transplanted hepatocytes isolated from normal (Wistar) donor rats into the Gunn rat. Considering the liver as the best acceptor organ, they infused a hepatocyte suspension, obtained by passing 2 grams of normal Wistar liver through a $140 \mu \mathrm{m}$ pore size metal screen, into the portal vein. After hepatocyte transplantation (HTX), all recipient rats received immunosuppressive therapy (rabbit antiserum to rat lymphocytes) to prevent rejection of the Wistar cells. A gradual fall in total plasma bilirubin was observed. Injection of Wistar hepatocytes into the peritoneum of Gunn rats did not result in declining plasma bilirubin. In control Gunn rats that received Wistar spleen cells into the portal circulation, a bilirubin reduction was not observed either. Groth (29) proved the reproducibility of these results. He transplanted a total of $25-35 \times 10^{6}$ ( $95 \%$ viable) Wistar hepatocytes, obtained by collagenase digestion of the donor liver, into the Gunn rat liver via the intraportal route. Another group of Gunn rats was treated by intramuscular transplantation. Intraportal HTX resulted 
in a decrease of total plasma bilirubin in Gunn rats, whereas intramuscular HTX was not successful.

In all the above reports, donor cell survival was poorly documented. Matas (28) reported that "preliminary histological studies" showed transplanted hepatocytes localized in the liver sinusoids, but he did not indicate the posttransplant interval of this observation. The survival of intraperitoneally dispersed hepatocytes was not mentioned. Sutherland (37) only considered the biological effects of HTX without referring to histological graft survival. Groth (29) found aggregates of well preserved hepatocytes in the distal portal branches and in the liver sinusoids at 10 days posttransplantation. However, after 5 weeks he could identify intravascular hepatocytes only occasionally, in spite of immunosuppression of the host rat.

The findings of these reports raised questions about the mechanism by which HTX reduced total plasma bilirubin levels in the Gunn rat. The explanation that bilirubin was conjugated by BGT activity in the donor hepatocytes and was subsequently excreted in its conjugated form into bile of the host, had never been supported by hard evidence. Several other possible mechanisms of action were postulated. In Woods' (38) opinion, metabolic activity of isolated hepatocytes would be inadequate for the conjugation of the amount of billirubin required to lower total plasma bilirubin in the Gunn rat. He proposed that infused cellular materials impaired the ability of the reticuloendothelial system to remove effete red blood cells, thereby prolonging red cell survival. Reduced hemoglobin catabolism and bilirubin synthesis would then explain the decline of total plasma bilirubin in the Gunn rat treated by HTX. In his experiments, he showed that not only HTX by direct infusion into the hepatic parenchyma (under an immunosuppressive regimen), but also splenectomy lowered total plasma bilirubin levels in the Gunn rat. In addition, he demonstrated prolongation of red cell survival following HTX. However, these observations were no definite proof for the proposed mechanism of action, as it might well concern a mere coincidence of events without causal relation. In the work of Rugstad (26), several mechanisms of action had been suggested that could also apply to HTX. First, the possibility was considered that genetic transformation occurred in the enzymedeficient liver through incorporation of informational macromolecules released from the transplanted hepatocytes. Second, it was proposed that a diffusible derepressor substance from the normal donor liver cells would enable the totally inactive genetic locus for BGT in the Gunn rat liver to function more efficiently.

The exact mechanism, by which HTX exerted its beneficial effect in enyzme deficiency states, was revealed by Vroemen et al. (this thesis, Chapter 2). He showed that surviving, metabolically intact donor hepatocytes were responsible for the production and appearance of the missing enzyme activity in the deficient host rat. Thereafter the same author showed in a newly developed rat model of congenital jaundice, consisting of two congenic deficient and non-deficient strains (see Chapter 3), that a long-term therapeutic effect could be obtained, when rejection of the donor hepatocytes did not occur (this thesis, Chapter 4). Very recently, Cobourn (39) showed that allogenic HTX into the spleen of the Gunn rat resulted in a sus- 
tained decrease of total plasma bilirubin under Cyclosporin A immunosuppression. The potential of HTX as a treatment modality for inherited metabolic disease was granted further credibility when Demetriou $(40,41)$ used HTX in the treatment of Nagase analbuminemic rats (NAR rats), which suffer from a congenital defect in albumin synthesis and whose plasma contains only traces of this protein. In these reports a new method of HTX was introduced. Isolated hepatocytes were attached to collagen coated dextran microcarriers and subsequently transplanted into the peritoneum. HTX with allogenic Wistar hepatocytes increased plasma albumin concentrations in the NAR rats from 0.5 grams/liter ( $g / 1$ ) to peak levels of $10 \mathrm{~g} / \mathrm{l}$ (in normal Wistar rats this value is $35-40 \mathrm{~g} / \mathrm{l}$ ) on the 6th posttransplant day. Hereafter these levels declined to the pretransplant level because of rejection of the donor cells. Cyclosporin A immunosuppression resulted in survival of the hepatocytes as assessed by light and scanning electron microscopic examinations. Under this immunosuppressive regimen, the therapeutic effect lasted during the entire test period (30 days) and a peak level of $13 \mathrm{~g} / 1$ was attained.

\section{Hepatocyte transplantation for acute hepatic failure.}

The fact that isolated liver cells appeared to be capable of replacing a single enzymatic function in an enzyme-deficient animal, initiated research to find if transplanted isolated hepatocytes could also perform other hepatospecific functions in the host, and provide metabolic support in other states of hepatic insufficiency, particularly in acute liver failure. As in enzyme deficiency disease, satisfactory management of this highly lethal condition does not exist, in spite of many experimental and clinical efforts including exchange transfusion, cross circulation, extracorporeal liver (or liver cell suspension) perfusion, and whole or auxilliary liver transplantation (42-48).

In 1977, HTX was applied for the first time in an animal model of acute liver failure by the same group of investigators that had demonstrated the beneficial effect of HTX in hereditary metabolic disease. Sutherland and co-workers (30) injected hepatocyte suspensions into the peritoneum and into the portal vein of syngenic rats that suffered acute hepatic failure. Liver failure had been induced by the administration of dimethylnitrosamine (DMNA) $24 \mathrm{hr}$ prior to HTX. DMNA is a rapidly metabolized and selectively hepatotoxic drug, which induces life-threatening hepatic failure, when given at an appropriate dose. In this model the mortality rate of untreated or sham-treated controls exceeded $80 \%$. In the $20 \%$ spontaneously surviving animals hepatic architecture and funtion were restored after approximately 3 weeks, showing that hepatic damage by DMNA was potentially reversible. In DMNApoisened rats treated by intraportal or intraperitoneal HTX mortality rates amounted to about $30 \%$ only. This positive effect of HTX was ascribed to life-sustaining metabollic support provided by donor hepatocytes during the critical period of liver regeneration. However, at histological examination intraportally transplanted hepatocytes were difficult to differentiate from the hosts own liver cells. 
A large portion of donor hepatocytes appeared to desintegrate within a few hours or days. In rats that received an intraperitoneal transplant, histological proof of graft survival could not be found. These findings did not exclude the possibility that microsomes or other subcellular fractions (or even cellular enzymes themselves) continued to function in the host after cytolysis, and were responsible for the increase in survival rates following HTX. Sommer (49) yielded similar results in rats with galactosamine induced liver failure. In this study, isolated hepatocytes were transplanted into the spleen, which appeared to be equally effective. $\mathrm{He}$ found that hepatocytes transplanted into the spleen survived, and did not seem to decrease in number. Moreover, in the spleen donor hepatocytes preserved their morphological cellular structure better than in the liver. Sommer $(31,50)$ also used this method of HTX in dogs with ischemia-induced liver failure. Autologous hepatocytes were prepared from the left lateral liver lobe (excised before the $45 \mathrm{~min}$. ischemic period), and were transplanted into the spleen at 48 hours before (31) and immediately after the ischemic insult ( 50$)$. In both settings, a significant increase in survival rates was observed compared to untreated controls (from $20 \%$ to $70 \%$ or more). Survival of intrasplenic hepatocytes was also observed in this dog model.

Intrasplenic and intraperitoneal HTX were also effective in a rat model of acute liver failure realized by a $70 \%$ partial hepatectomy combined with a portacaval shunt (51). Intact intrasplenic hepatocytes were demonstrated in this report. There was no mention of donor hepatocyte survival in the peritoneal cavity.

The efficacy of treatment with syngenic liver cells seemed to be time-related $(52,53)$. In some series highest survival rates were obtained when HTX was performed at 48-60 hours after induction of acute hepatic failure, whereas in the experience of others, syngenic HTX was effective only when applied within 24 hours after liver injury. The use of different models of acute liver failure probably accounts for these diverging findings, and render comparison of results between various reports extremely difficult.

In a series of publications, Makowka $(32,53,54)$ demonstrated elegantly that not only transplantation of syngenic, but also of allogenic (across minor and major histocompatibility barriers) and xenogenic (rabbit and porcine) hepatocytes resulted in significantly reduced mortality rates in galactosamine poisoned rats. By in vitro and in viwo assessment of immunological responses of intoxicated rats to allogenic and xenogenic HTX, he ruled out the possibility of an immunosuppressive effect of galactosamine that would have permitted the survival of allo- or xenogenic cells $(53,54)$. Recipients of such cells appeared to be sensitized after repeated infusions, but sensitization did not abrogate the therapeutic influence of allo- or xenogenic HTX. Surviving donor hepatocytes were never encountered in the peritoneal cavity. The use of irradiated (non-proliferative) hepatocytes or cryopreserved hepatocytes with limited viability did not negatively influence the results either $(32,53)$. Furthermore, by transplanting ${ }^{3} \mathrm{H}$-thymidine labeled liver cells, it was demonstrated that donor cell repopulation of the recipient liver did not occur (32). These results strongly suggested that true metabolic support provided by intact, metabolically active 
donor hepatocytes did not account for the improved cure rates in acute liver failure, but that a cellular "trophic" compound was involved that stimulated regeneration of the injured liver. This supposition was verified by administering cytosol extracts of liver to galactosamine poisoned rats. Makowka (55) reported an identical improvement of survival rates as observed in HTX-treated rats. These findings were extended by LaPlante O'Neill (56) and Ohkawa (57), who achieved similar positive results using cell-free supernatants from hepatocyte cultures and cytosol fractions from regenerating liver, respectively. These studies proved that surviving hepatocytes are not essential for the successful treatment of acute liver failure. As a consequence, a protocol of allogenic HTX in the treatment of human fulminant liver failure, that was initiated at Makowka's institution after his initial promising results $(32,53)$, was changed in favour of the technique using a cytosol fraction (55). As yet, only preliminary results of clinical HTX or liver cytosol treatment are available. Five patients with fulminant hepatic failure received intraperitoneal injections of porcine hepatocyte suspensions (one patient), human hepatocyte suspensions (one patient) and human liver cytosol fractions ( 3 patients). A transient improvement was noticed in two patients. Untoward effects of this treatment were not observed. However, all five patients died (58).

\section{Morphologic and functional studies of hepatocellular grafts.}

The promising results of experimental HTX in the treatment of partial or total liver insufficiency stimulated investigators to perform specific research into morphologic, cytokinetic and metabolic aspects of HTX.

At first sight, the liver would seem the most appropriate site for inoculation of the isolated hepatocytes, because of the "natural" environment and the supply of portal blood, thought to be necessary for sustained viability of liver cells (59). In order to determine the most suitable location for the survival of isolated hepatocytes, Ebata performed syngenic HTX into the spleen, kidney, liver and peritoneal cavity. Viable donor hepatocytes could only be identified in the spleen after transplantation. Also in earlier mentioned studies $(49,51)$, the spleen seemed to be a suitable acceptor organ. In the experience of others, it was difficult or even impossible to differentiate donor hepatocytes (transplanted into the liver by infusion into the portal venous system) from the host's own liver cells $(30,60,61)$. It was shown by the author of this thesis that the pancreas was another suitable location for the nidation of isolated hepatocytes. However, no additional advantages were observed in comparison to intrasplenic HTX (this thesis, Chapter 5). Most research groups, including those who initially performed HTX via the portal system or into the intra-abdominal cavity, have opted now for the spleen as the preferred acceptor site.

Mito (60) studied the spleen of rats one year after syngenic HTX. He found that intrasplenic HTX led to "hepatization" of the spleen. The morphological structure of the liver parenchymal cells remained intact, and was identical to that of normal hepatocytes. As in normal rat liver, many hepatocytes were binuclear, and mitotic 
figures were not observed. Histochemical studies showed that the intrasplenic hepatocytes continued to synthesize albumin and glycogen. The transplanted hepatocytes formed aggregates in the red pulp of the spleen. Formation of hepatic cords and sinusoidal spaces was visible. The amount of hepatic tissue found in these spleens suggested that proliferation of the transplanted hepatocytes occurred. At 14-17 months post-HTX, up to $40 \%$ of splenic surface area was occupied by hepatic tissue. Surprisingly, spleen weight did not increase, but even decreased after HTX. It is not impossible that declining spleen mass after HTX contributed to the impression that donor hepatic tissue slowly increased. Electron microscopic studies performed by Mito's group (60-62) demonstrated the formation of bile canaliculi and desmosomes between adjacent donor hepatocytes, as well as sinusoidal spaces lined by endothelium. In the cytoplasm of the liver cells, mitochondria and normal endoplasmatic reticulum were seen. Glycogen particles were present, although in decreased number compared to non-transplanted cells.

Based on morphological observations at various posttransplant intervals, Kusano and Mito postulated that the number of injected hepatocytes decreases in the first few weeks post-HTX. They assumed that in this period vulnerable, damaged or nutritionally deprived cells would degenerate, become necrotic and eventually disappear. Remaining transplanted cells would start to proliferate and slowly reconfigurate (partially at least) the hepatic microstructure (62). Recently, it has been shown by autoradiographic studies that proliferation of transplanted hepatocytes was present in the first week, but seemed absent in the second week (63). Vroemen et al. investigated the spontaneous cytokinetic behaviour of hepatocytes after transplantation into the spleen, and showed that these ectopic liver cells indeed exhibited mitotic activity up to at least 20 weeks post-HTX (this thesis, Chapter 6). It is worth mentioning, that intrasplenic transplantation of syngenic hepatocytes, obtained from cirrhotic rats, seemed to result in accelerated proliferation and a more rapid formation of the hepatic cord structures (61). Accelerated growth of intrasplenic liver mass was observed also after simultaneous partial hepatectomy and HTX with preneoplastic liver cells (64), or with normal hepatocytes (65), when the proliferative response of the remaining host liver was drug-inhibited. These lastmentioned studies suggest that modulation of the proliferative process of transplanted hepatocytes may be feasible.

Also the functional consequences of HTX were studied. The values of serum alphafetoprotein in HTX-treated rats appeared to be normal $(20 \mathrm{ng} / \mathrm{ml})(60)$. Ammonia tolerance tests demonstrated that there was a significantly better clearance of ammonia in rats with a portacaval shunt and "hepatized" spleens, than in rats with a portacaval shunt without hepatized spleens (60). Indocyanine green clearance tests revealed similar findings (60). Cuervas-Mons $(66,67)$ and Woods $(68)$ assessed metabolic activity of donor hepatocytes by means of ex vivo ${ }^{99 m}$ Tc-HIDA scintigraphy of HTX-treated spleens. The first author performed his study using syngenic HTX in normal rats. Histological and scintigraphic investigations were conducted weekly during the first month, and at 11 monthly intervals thereafter. He demonstrated that 
gamma-activity measured in the excised hepatized spleens, 10 minutes after i.v. administration of ${ }^{99 \mathrm{~m}} \mathrm{Tc}-\mathrm{HIDA}$ to the recipient, was significantly higher than in spleens of untreated control rats. The amount of radioactivity measured in the hepatized spleens at the various posttransplant intervals, however, remained constant during the entire study. In the histological part of the study, Cuervas-Mons described similar findings as Mito, in particular the formation of hepatic cell cords, but he never found any evidence of hepatocellular proliferation (67). This was in agreement with the results of his scintigraphic studies, in which he could not find arguments for the growth of intrasplenic hepatic mass either. In Woods' study (68), graft survival was not observed after syngenic HTX, only after autologous HTX. The author attributed this to immunological rejection, assuming that the allegedly inbred rat strain used in his study still exhibited individual genetic variation. As in the preceding study, radioactivity measured in hepatized spleens (more than 7 weeks post-HTX) $45 \mathrm{~min}$. after ${ }^{99 m}$ Tc-HIDA administration was significantly higher than in spleens from untreated controls. However, proliferative activity of donor liver cells was not demonstrable in his material. From the spleens containing autologous hepatocytes (10-35 weeks postHTX), both bilirubin mono- and diglucuronide were recovered 30 minutes after parenteral administration of $35 \mathrm{mg}$ unconjugated bilirubin. In control spleens these molecules were not detected. In contrast to the previous postmortem scintigraphic ${ }^{99 m}$ Tc-HIDA study, Vroemen et al. applied in vivo dynamic ${ }^{99 m}$ Tc-HIDA scintigraphy for the monitoring of graft function after intrasplenic HTX (this thesis, Chapter 7).

This overview shows that isolated liver cells retain many, if not all, hepatospecific functions after transplantation. An appropriate acceptor environment for nidation and survival of the hepatocytes seems to be required. Preliminary results of HTX, in particular in animal models of enzyme deficiency, are encouraging and may offer perspectives for clinical use in the future.

\section{Introduction to the experimental work}

When the research activities underlying this thesis were started in 1982 , only few reports on hepatocyte transplantation (HTX) were published, and a generally accepted technique of HTX was not available. Consequently, the first objective was to acquire the appropriate techniques for the isolation and transplantation of hepatocytes.

The collagenase digestive procedure was chosen for the isolation of rat liver cells as it possessed obvious advantages compared to the mechanical isolation method. Both cell yield and cell viability were higher, and metabolic capacities appeared to be better preserved using the collagenase perfusion method $(34,38)$. The (slightly modified) method of Seglen $(69,70)$, which differed only in details from the original method of Berry and Friend (71), was used in our experiments. This method is described in more detail in the following chapters. After filtration of the initial cell 
suspension, obtained after enzymatic digestion of the liver collagen matrix, the cells were washed (at $0-4^{\circ} \mathrm{C}$ ) three to four times by low gravity "differential" centrifugation (25-50 g during 2 minutes). In this manner, cellular debris and nonparenchymal cells (endothelial and Kupfer cells) were separated from the parenchymal cells. After the last washing procedure, the cellular pellet (consisting of $95 \%$ parenchymal cells) was suspended to the required concentration. The resulting hepatocyte suspension was injected into the spleen of the recipient rat. Hepatocyte survival in the recipient rats was tested in a syngenic Wistar strain. After this primary goal had been attained, the rat model of congenital jaundice was used for further research into HTX.

The aim of the first study, which is presented in Chapter 2, was to unravel the exact mechanism of action of HTX in the treatment of enzyme deficiency disease. Knowledge of this mechanism would provide the proper basis for further research in this field. We transplanted allogenic hepatocytes from normal Wistar rats into the spleen of Gunn rats. A reduction of plasma bilirubin levels in the recipients was obtained, although it was only transitory. Rejection of the Wistar donor hepatocytes was found to be responsible for this transient beneficial effect. In order to assess whether transplanted hepatocytes provided the missing enzyme activity in the Gunn rat, bile samples from HTX-treated Gunn rats were analyzed for the presence of bilirubin glucuronides, the products of the deficient enzyme. The results of these analyses, in combination with the histological data in this study, revealed the mechanism of action of HTX.

In order to avoid rejection, two congenic, histocompatible rat strains were needed, one of which was enzyme-deficient. A new rat strain with hereditary unconjugated hyperbilirubinemia was developed which was congenic with a normal Wistar strain. This was achieved by repeated backcross-intercross breeding of the Gunn rat strain (as donor of the jaundiced trait) with an inbred normal Wistar rat strain at the Laboratory Animal Centre ('Proefdierencentrum") of the University of Leuven, Belgium. The resulting congenic rat strain was tested on biochemical properties, histocompatibility and growth characteristics. Furthermore, HTX was performed in this rat strain to assess its suitability for further research into this transplantation procedure. A detailed description of the development of the new congenic strain and of the results of histocompatibility testing and biochemical characterization is provided in Chapter 3.

The results of the preceding work raised the question whether a long-term therapeutic effect of HTX could be obtained in enzyme deficiency disease. This issue was subject of investigation in Chapter 4 . The assessment of long-term metabolic activity of hepatocytes after transplantation was of crucial importance, as it was known that isolated hepatocytes cultured in vitro rapidly loose their metabolic capacities (34-36). The availability of the above-mentioned histocompatible rat strains allowed us to obtain absolute proof for the persisting metabolic function(s) of the normal 
donor hepatocytes by demonstrating the continuing presence of the previously lacking enzyme activity in the HTX-treated jaundiced rats. In this experimental work, insight was also gained into the anatomical distribution of the isolated liver cells after transplantation.

In Chapter 5, the pancreas was tested as an acceptor organ for isolated hepatocytes. Until then, the spleen was the preferred acceptor organ for the transplantation of isolated hepatocytes. The embryological origin of the pancreas, and the presence of so-called hepatotrophic factors contained in it, provided a rational basis to presume that the pancreas would also supply the appropriate environment for the survivall and continuing metabolic activity of a hepatocellular transplant $(72,73)$. The suitability of the pancreas as an acceptor organ for hepatocytes was tested in the rat model that was described in Chapter 3. The experimental protocol was designed to allow comparison between the results of intrasplenic and intrapancreatic HTX, in respect to the therapeutic effect obtained.

Chapter 6 contains a study that focusses on the proliferative activity of transplanted hepatocytes. On the basis of results obtained in foregoing chapters, it was assumed that the therapeutic effect provided by the hepatocellular graft in enzyme deficiency disease correlated positively to the total amount of functional donor hepatocytes in the host. Therefore, knowledge of the cytokinetic behaviour of the donor hepatocytes was of great interest. The scarce data in the literature on the subject of proliferative behaviour of the intrasplenic donor hepatocytes were not conclusive and sometimes confusing or even conflicting. In the present experiments, a nonautoradiographic method was used for the detection of S-phase cells in histological sections, in order to evaluate the presence of spontaneous proliferative activity of intrasplenic donor hepatocytes. The effect of partial hepatectomy in the host on the proliferation index of the donor cells was also investigated. An enzymologic method for the quantification of liver mass contained in the spleen was developed. This method permitted us to relate observed proliferative activity of transplanted hepatocytes to actual alterations of intrasplenic liver mass.

In the previous chapters, donor hepatocyte survival was monitored by histological examination of the spleen of the HTX-treated rat. Intact metabolic activity of normal hepatocytes grafted in the spleen of BGT-deficient rats was verified by the analysis of the recipient's bile on the presence of glucuronized bilirubins. Both methods of graft function monitoring implied death of the recipient, and did not allow repeated investigations in the individual animal. It had been demonstrated by other investigators, that the intrasplenic hepatocytes accumulated 99mTc-HIDA (66-68). In these studies, measurements of ${ }^{99 \mathrm{~m}} \mathrm{Tc}$-HIDA uptake in the spleen were performed ex vivo. The results in the literature suggested that in vivo ${ }^{99 m} \mathrm{Tc}-\mathrm{HIDA}$ scanning would be feasible. In vivo dynamic $99 \mathrm{~m}$ Tc-HIDA scintigraphy in HTXtreated rats would provide two major advantages compared to the "classic" 
methods of graft function monitoring; first, scintigraphic studies would not require autopsy, and thus would permit, in principle, repeated measurements in the individual animal. Second, not only survival could be verified, but also the kinetics of hepatocellular ${ }^{5}$ miTc-HIDA uptake (to be considered as a parameter of enzymatic processes) could be studied by means of this technique. Chapter 7 deals with the results of dynamic ${ }^{9 \%}$ Tc-HIDA scanning in HTX-treated rats. Scintigraphic data were interpreted in the light of concomitant histological and biochemical investigations.

\section{References to the Preface and Chapter 1.}

1. Rosenberg LE.

Metabolic diseases: future directions.

In: Birth defects. Proceedings of the fifth international conference on birth defects. Edited by: Littlefield JW and de Grouchy J. Excerpta Medica, Amsterdam, 1978; p. 364.

2. Benson PF, Fension AH.

Genetic biochemical disorders.

Edited by: Bobrow M, Harper PS, Motulsky AG. Oxford University Press, Oxford, 1985.

3. Stanbury JB, Wijngaarden JB, Fredrickson DS, Goldstein JL, Brown MS.

Inborn errors of metabolism in the 1980s.

In: The metabolic basis of inherited disease. Edited by: Stanbury JB, Wijngaarden JB, Fredrickson DS, Goldstein JL, Brown MS. MeGraw-Hill Book Company, New York, 1983; p. 33.

4. Desnick RJ.

Treatment of inherited metabolic diseases: Current status and prospects.

In: Genetic disease. Diagnosis and treatment. Edited by: Dietz AA. The American Association for Clinical Chemistry, Washington, 1983 ; p. 183.

5. Anderson WF.

Prospects for human gene therapy.

Sience $1984 ; 226: 401$.

6. Weatherall DJ.

DNA in medicine: Implications for medical practice and human biology.

Lancet $1984 ; 2: 1440$.

7. Culliton BJ.

Gene therapy: research in public.

Science 1985; 227: 493.

8. Clayton $\mathrm{BC}$.

The principles of treatment by dietary restriction as illustrated by phenylketonuria.

In: The treatment of inherited netabolic disease. Edited by: Raine DN. Medical Technical Publishing $\mathrm{Co}_{1}$ Lancaster, $1975 ; \mathrm{p} .1$.

9. Brady RO, Tallman JF, Johnson WG et al.

Replacement therapy for inherited enzyme deficiency. Use of purified ceramidetrihexosidase in Fabry's disease.

N Engl J Med 1973; 289: 9.

10. Brady RO, Pentehev PG, Gal AE "Hibbert SR, Dekaban AS.

Replacement therapy for inherited enzyme deficiency. Use of purified glucocerebrosidase in Gaucher"s disease.

N Engl J Med 1974; $291: 989$.

11. Johnson WG, Desnick RJ, Long DM et al.

Intravenous injection of purified hexosaminidase $\mathrm{A}$ into a patient with Tay-Sachs disease.

Birth defects: Original article series $1973 ; 9: 120$.

12. Starzl TE.

The ripple effect of liver transplantation.

Transplant Proc 1980; 12: 626. 
13. Sutherland DER, Matas AJ, Najarian JS.

The mutual impact of transplantation and advances in the understanding and treament of metabolic diseases.

Transplant Proc 1980; 12: 643.

14. Machioro TL, Hougie C, Ragde H, Epstein RB, Thomas ED.

Hemophilia: role of organ homografts.

Science 1969; 163: 188.

15. Calne $R Y$, Williams $R$.

Liver transplantation.

Curr Prob Surg 1979; 16: 1.

16. Groth CG, Dubois RS, Corman J et al,

Starzl TE.

Metabolic effects of hepatic replacement in Wilson's disease..

Transplant Proc 1973; 5: 829.

17. Dalloze $\mathbf{P}$, Corman J, Bloch $\mathrm{P}$, Delvin EE, Glorieux FH.

Enzyme replacement in Niemann-Pick disease by liver homotransplantation.

Transplant Proc 1975; 7: 607.

18. MacDougall $\mathbf{B R D}$, Williams $\mathbf{R}$.

The indications for orthotopic liver transplantation.

Transplant Proc 1979; 11: 247.

19. Hoitsma HFW, Meijer S, Rauwerda JA, Stanenkowic L, de Lange JJ, Vermulst ALFJ.

Indicaties voor orthotope levertransplantatie.

Ned Tijdsch Geneesk 1980; 124: 556.

20. Hood JM, Koep LJ, Peters RL et al.

Liver transplantation for advanced liver disease with alpha-1-antitrypsine deficiency.

N Engl J Med 1980; 302: 272.

21. Zitelli BJ, Malatack JJ, Gartner JC, Shaw BW, Iwatsuki S, Starzl TE.

Orthotopic liver transplantation in children with hepatic-based metabolic disease.

Transplant Proc 1983; 15: 1284.

22. Starzl TE, Iwatsuki S, Shaw B.

Current status of liver tranplantation.

In: Current status of clinical organ transplantation. Edited by: Abouna GM, White AG. Martinus

Nijhoff Publishers, Boston 1984; p. 261.

23. Wolff $H$, Otto $G$, Giest $H$.

Liver transplantation in Crigler-Najjar syndrome. A case report.

Transplantation 1986; $42: 84$.

24. Starzl TE, Iwatsuki S, Shaw BW, Gordon RD.

Liver. Orthotopic liver transplantation in 1984.

Transplant Proc 1985; 17: 250.

25. Gunn $\mathrm{CM}$.

Hereditary acholuric jaundice in a new mutant strain of rats.

J Hered 1938; 29: 137.

26. Rugstad $\mathrm{HE}$, Robinson $\mathrm{SH}$, Yannoni C, Tashjian $\mathrm{AH}$.

Transfer of bilirubin uridine diphosphate-glucuronyl transferase to enzyme-deficient rats.

Science 1970; 170: 553 .

27. Mukherjee AB, Krasner J.

Induction of an enzyme in genetically deficient rats after grafting of normal liwer.

Science $1973 ; 182 ; 68$.

28. Matas AJ, Sutherland DER, Steffes MW et al.

Hepatocellular transplantation for metabolic deficiencies: decrease of plasma bilirubin in Gunn rats.

Science 1976; 192: 892 .

29. Groth CG, Arborgh B, Björkến C, Sundberg B, Lundgren $G$.

Correction of hyperbilirubinaemia in the glucuronyl-transferasedeficient rat by intraportal hepato. cyte transplantation.

Transplant Proc 1977; 9: 313 . 
30. Sutherland DER, Numata M, Matas AJ, Simmons RL, Najarian JS.

Hepatocellular transplantation in acute liver failure.

Surgery $1977: 82: 124$.

31. Sommer BG, Sutherland DER, Simmons RR, Najarian JS.

Hepatocellular transplantation for acute liver failure in dogs.

Surg Forum 1979; 30: 279.

32. Makowka L, Falk RE, Rotstein LE et al.

Cellular transplantation in the treatment of experimental hepatic failure.

Science 1980; $210: 901$.

33. Scragg 1, Celier C, Burchell B.

Congenital jaundice in rats due to the absence of hepatic bilirubin UDP-glucuronyltransferase enzyme protein.

FEBS letters 1985; 183: 37 .

34. Drochmans P, Wanson JC, May C, Bernaeri D.

Ultrastructural and metabolic studies of isolated and cultured hepatocytes.

In: Hepatotrophic factors. Ciba Foundation Symposium 55. Edited by: Porter R, Whelan J. Elsevier/Excerpta Medica/North-Holland, Amsterdam 1978; p. 7.

35. Bissel DM.

Study of thepatocyte function in cell culture.

In: Progress in liver diseases. Edited by: Popper $H$, Schaffner F. Grune and Stratton, New York 1976; p. 69.

36. Gugnen-Guillouzo $C_{n}$, Guillowzo A.

Modulation of functional activities in cultured rat hepatocytes.

Mol Cell Biochem 1983; 53: 35.

37. Sutherland DER, Matas AJ, Steffes MW, Simmons RL, Najarian JS.

Transplantation of liwer cells in an animal model of congenital enzyme deficiency disease: the Gunn rat.

Transplant Proc 1977; 9: 317.

38. Woods RJ, Parbhoo SP.

An explanation for the reduction in bilirubin $\Downarrow$ evels in congenitally jaundiced Gumn rats after transplantation of isolated hepatocytes.

Eur Surg Res 1981; 13: 278.

39. Cobourn CS, Makowka L, Falk JA, Falk RE.

Allogeneic intrasplenic hepatocyte transplantation in the Gunn rat using Cyclosporine $A$ immunosuppression. (Abstract).

Presented at the 11th International Congress of the Transplantation Society, Helsinki, 1986.

40. Demetriou AA, Whiting JF, Feldman $\mathrm{D}$ et al.

Chowdhury JR.

Replacement of liver function in rats by transplantation of microcarrier-attached hepatocytes.

Science 1986; 233: 1190.

41. Demetriou AA, Whiting JF, Levenson $S M$ et al.

D, Chowdhury $\mathbf{J R}$.

New method of hepatocyte transplantation and extracorporeal liver support.

Ann Surg 1986: 204: 259 .

42. Redeker $\mathrm{AG}$, Yamahiro HS.

Controlled trial of exchange-transfusion therapy in fulminant hepatitis.

Lancet 1973; I: 3.

43. Santiago-Delpin EA, Callender $\mathrm{C}$, Kjellstrand $\mathrm{CM}$.

Cross-dialysis in the treatment of experimental fulminant hepatic coma.

J Surg Res 1975; 19: 175.

44. Silk DBA, Trewby PN, Chase RA et al.

Treatment of fulminant hepatic failure by polyacrylonitrile-membrane haemodialysis.

Lancet $1977 ; 1: 1$.

45. Eisenan $B$, Liem DS, Raffucei $F$.

Heterologous liver perfusion in treatment of hepatic failure.

Ann Surg 1965; 162: 329. 
46. Abouna GM, Boehm HG, Serrou B, Amemiya $\mathrm{H}$, Martineau G.

Longterm hepatic support by intermittent multi-species liver perfusions.

Lancet 1970; 1: 391.

47. Olumide F, Eliashiv A, Kralios N, Norton L, Eiseman B.

Hepatic support with hepatocyte suspensions in a permeable membrane dialyzer.

Surgery 1977; 82: 599 .

48. Diaz A, Ricco JB, Franco D, Gigou M, Szekely A, Bismuth H.

Temporary liver transplantation in acute liver failure.

Arch Surg 1977; 112: 74.

49. Sommer BG, Sutherland DER, Matas AJ, Simmons RL, Najarian JS.

Hepatocellular transplantation for treatment of galactosamine-induced acute liver failure in rats.

Transplant Proc 1979; 11: 578.

50. Sommer BG, Sutherland DER, Simmons RL, Najarian JS.

Hepatocellular transplantation for experimental ischemic acute liver failure in dogs.

J Surg Res 1980; 29: 319.

51. Minato M, Houssin D, Demma I et al.

Transplantation of hepatocytes for treatment of surgically induced hepatic failure in the rat.

Eur Surg Res 1984; 16: 162.

52. Cuervas-Mons V, Cienfuegos JA, Maganto $P$ et al.

Time-related efficacy of liver cell isografts in fulminant hepatic failure.

Transplantation 1984; 38: 23.

53. Makowka $\mathbb{L}$, Rotstein LE, Falk RE et al.

Reversal of toxic and anoxic induced hepatic failure by syngeneic, allogeneic, and xenogeneic hepatocyte transplantation.

Surgery $1980 ; 88: 244$.

54. Makowka L, Rotstein LE, Falk RE et al.

Allogeneic and xenogeneic hepatocyte transplantation in experimental hepatic failure.

Transplantation 1980;30: 429 .

55. Makowka L, Rotstein LE, Falk RE et al.

Studies into the mechanism of reversal of experimental acute hepatic failure by hepatocyte transplantation.

Can J Surg 1981; 24: 39.

56. La Plante O'Neill P, Baumgartner D, Lewis WI, Zweber BA, Sutherland DER.

Cell-free supernatant from hepatocyte cultures improves surwival of rats with chemically induced acute liver failure.

J Surg Res 1982; 32: 347.

57. Ohkawa $M$, Hayashi $H$, Chaudry IH, Clemens MG, Baue AE.

Effects of regenerating liver cytosol on drug-induced hepatic failure.

Surgery $1985 ; 97: 455$.

58. Makowka L, Falk RE, Blendis LM et al.

The use of liver cytosol fractions in the treatment of acute liver failure.

(submitted for publication).

59. Starzl TE, Porter KA, Francavilla JA, Benichou J, Putnam CW.

A hundred years of the hepatotrophic controversy.

In: Hepatotrophic factors. Ciba Foundation Symposium 55. Edited by: Porter R, Whelan J. Elsevier/Excerpta Medica/North-Holland, Amsterdam 1978; p. 111.

60. Mito M, Ebata H, Kusano M, Onishì T, Saito T, Sakamoto S.

Morphology and function of isolated hepatocytes transplanted into rat spleen.

Transplantation 1979; 28: 499.

61. Mito M, Ebata H, Kusano M, Onishi T, Hiratsaka M, Saito T.

Studies on ectopic liver utilizing hepatocyte transplantation into the rat spleen.

Transplant Proc 1979; 11: 585.

62. Kusano M, Mito $M$.

Observations on the fine structure of longsurvived isolated hepatocytes inoculated into rat spleen.

Gastroenterology 1982;82:616.

63. Nordlinger $B$, Wang $S R$, Bouma ME et al. 
Can hepatocytes regenerate when transplanted in the spleen?

(Ábstract)

Eur Surn Res 1986; 18(S1): 104.

64. Finkelstein SD, Lee $\mathrm{G}_{*}$ Medine $A$, Tatematsu $M$, Makowka $\mathbb{L}$, Farber $E$.

An experimental method for rapid growth of liver in spleen. The survival and proliferation of chemically induced preneoplastic hepatocytes in spleen.

Am JPatholl 1983; 110: 119.

65. Makowka L, Lee G, Cobourn CS, Farber E, Falk JA, Falk RE.

Allogeneic hepatocyte transplantation in the rat spleen under cyclosporine immunosuppression.

Transplantation 1986; 42:537.

66. Cuervas-Mons V, Maganto $P$, Cienfuegos JA et al.

Morphology and ${ }^{95 \mathrm{~m}}$ TC-HIDA functional study of isolated syngeneic hepatocytes transplanted into rat spleen. (Abstract)

Eur Surg Res 1981: 13(S1): 60.

67. Cuervas-Mons V, Cienfuegos JA, Maganto P, Rodriguez V, Eroles G, Pinedo I, Santamaria L, Ramos J, Ortiz JL, Castillo-Olivares $\mathrm{JL}_{\text {, Segovia J. }}$

Long-term evaluation of isolated syngeneic hepatocytes transplanted into the normal rat spleen by TC-99M-HIDA scintigraphy.

Transplantation $1985 ; 39: 87$.

68. Woods RJ, Fuller BJ, Attenburrow VD, Nutt LH, Hobbs KEF.

Functional assessment of hepatocytes after transplantation into rat spleen.

Transplantation 1982; 33: 123.

69. Seglen PO.

Preparation of isolated rat liver cells.

Methods Cell Biol 1976; 13: 29.

70. Seglen PO.

Preparation of rat liver cells. Ill. Enzymatic requirements for tissue dispersion.

Exp Cell Research 1973; 82: 391 .

71. Berry MN, Friend DS.

High-yield preparation of isolated rat liver parenchymal cells. A biochemical and fine structural study.

J Cell Bioll 1969; 43: 506.

72. Leffert HL, Koch KS, Moran T, Rubalcava B.

Hormonal controll of rat liver regeneration.

Gastroenterology 1979; 76: 1470.

73. Bucher NLR, Patel U, Cohen S.

Hormonal factors concerned with liver regemeration.

In: Hepatotrophic factors. Ciba Foundation Symposium 55. Edited by: Porter R, Whelan J. Elsevier/Excerpta Medica/North-Holland, Amsterdam 1978; p. 95. 


\section{CHAPTER 2}

\section{Treatment of enzyme deficiency by hepatocyte transplantation in rats}

\section{Summary}

The inbred, homozygous Gunn rat exhibits unconjugated hyperbilirubinemia due to a hereditary absolute deficiency of bilirubin UDP-glucuronyl transferase activity. The mechanism of action of hepatocyte transplantation (HTX) in the treatment of enzyme deficiency has been investigated in this study.

Gunn rats underwent HTX by the injection of isolated hepatocytes from a nondeficient donor rat (Wistar) into the spleen. A transient, statistically significant decrease in total plasma bilirubin (TB) levels was observed. Gunn rats receiving Gunn hepatocytes did not show such decrease. Histological examination 2-3 months postHTX of the recipient spleens showed the absence of grafted hepatocytes in the first group and graft survival in the second. Bile specimens from sublethal irradiated Gunn rats, collected 6 days after HTX with viable Wistar hepatocytes, all contained bilirubin mono- and diglucuronides. Control groups consisting of Gunn rats receiving nonviable Wistar hepatocytes or Gunn hepatocytes, and sham-operated Gunn rats did not excrete bilirubin glucuronides in bile. It was also demonstrated that bilirubin UDP-glucuronyl transferase activity, which appeared in Gunn rats after HTX with Wistar hepatocytes, was only transient.

It is concluded that the decrease of TB in the Gunn rat after HTX with nondeficient hepatocytes is explained by the appearance of the enzyme, which was absent in the recipient animal. Viable, nondeficient hepatocytes are required for the elicited bilirubin conjugation. Rejection of the transplanted hepatocytes abolishes this effect. 


\section{Introduction}

Heriditary enzyme deficiencies remain a difficult therapeutic problem. Medical enzyme replacement therapy as well as whole organ transplantation, e.g., liver and kidney, have been attempted clinically as treatment for some of these diseases without entirely satisfying results $(1,2,3)$. Recent experimental work shows that transplantation of isolated hepatocytes may offer new perspectives for the treatment of heriditary liver enzyme deficiencies $(4,5,6)$.

The homozygous Gunn rat, a mutant strain of Wistar origin, represents an animal model for such enzyme deficiency, exhibiting unconjugated hyperbilirubinemia due to a hereditary absolute deficiency of hepatic bilirubin UDP-glucuronyl transferase activity (7). This enzyme deficiency disease is known in the human as the CriglerNajjar syndrome. It has been shown that intraportal hepatocyte transplantation (HTX) with cells from nondeficient donors causes a decrease of total plasma bilirubin (TB) in immunosuppressed Gunn rats $(5,4,6)$. The exact mechanism of action of HTX in the treatment of enzyme deficiency is still uncertain.

We investigated whether or not the decrease in TB following HTX is associated with the appearance of the enzyme activity, which was absent in the recipient Gunn rat, and of which factor(s) this phenomenon is dependent. Our present findings show that the reduction of TB after HTX with viable nondeficient hepatocytes is associated with the excretion in bile of bilirubin glucuronides. Bilirubin glucuronidation is neither observed in bile of non- or sham-treated Gunn rats, nor in Gunn rats treated with Gunn hepatocytes, or with nonviable Wistar hepatocytes. The induced bilirubin glucuronidation is abrogated by the rejection of transplanted allogenic hepatocytes, indicating that survival of transplanted nondeficient hepatocytes is necessary for the elicited conjugation.

\section{Materials and methods}

\section{Animals}

Inbred male homozygous GUNNXR/Pfd-j/j rats and inbred male Wistar R/APfd rats (200-300 g, purchased from the Proefdierencentrum, University of Leuven, Leuven, Belgium) were used as hepatocyte recipients and donors in this study. All animals were maintained on standlard laboratory chow and water ad libitum.

\section{Isolation of hepatocytes}

Isolation of hepatocytes was performed with the two-step collagenase digestive method as described by Seglen (8). The initial cell suspension obtained after collagenase digestion of the liver collagen matrix was filtered through $100-\mu \mathrm{m}$ nylon mesh to eliminate cell clusters, and washed three times in Krebs-Henseleit solution 
containing $10 \mathrm{mg} / \mathrm{ml}$ fatty-acid-free bovine serum albumin. This isolation method resulted in an average yield of $2-4 \times 10^{8}$ hepatocytes per liver, with a viability of 85-95\% (trypan blue exclusion test). The cells were resuspended to a concentration of $10^{7}$ viable cells per $\mathrm{ml}$ in Krebs-Henseleit solution and stored on ice. Hepatocytes were transplanted within 1 to $4 \mathrm{hr}$ after isolation. Cell viability was not adversely affected during this period of cold storage.

\section{Hepatocyte transplantation}

The spleen of the recipient rat was exteriorized under ether anesthesia through a left subcostall incision. One $\mathrm{ml}$ of the hepatocyte suspension containing $10^{7}$ viable cells was slowly injected into the splenic pulp by means of a 25 -gauge needle. Hilar vessels of the spleen were not clamped. Hemostasis was performed by compression of the injection site with a cotton-tip.

\section{Permanent bile fistula}

A modification of the technique described by Vonck (9) was used, which permitted normal bile flow into the duodenum. Under Nembutal anesthesia a midline abdominal incision was performed, and two silicone cannulae were inserted $5 \mathrm{~mm}$ into the common bile duct; one in the proximal direction (the afferent loop), the other in the distal direction (the efferent loop). The cannulae were fixed to the common bile duct and the abdominal wall. Both loops were tunneled subcutaneously over the left thoracic wall to the neck of the animal. The abdomen was closed. The ends of both loops were exteriorized through a midline incision over the skull and connected to a short bent piece of stainless-steel tubing. The two metal tubes were fixed to the skull by means of three 3-mm stainless-steel screws and a nontoxic dental expoxy resin. The metal tubes were connected by a $U$ loop of polyethylene tubing (PE-100) to reestablish normal bile flow into the distal bile duct. Postoperatively the patency of the system was checked daily by examination of the outflow of bile from the afferent loop, and by flushing the distal loop with a physiological saline solution. The connection of PE-100 tubing to the afferent metal tube allowed bile sampling from the unanesthetized, freely moving animal.

\section{Analytical methods}

TB of all blood samples was measured with the diazo method of Jendrassik-Grof. $T B$ levels in the different experimental groups were compared using the Wilcoxon rank test.

Bile of the transplanted animals was examined for the presence of bilirubin glucuronides by a combination of several analytical methods and structure elucidation procedures. First, the alkaline methanolysis method combined with highperformance liquid chromatography as described by Blanckaert et al. (10) was used 
to determine the presence and quantity of bilirubin monoester and diester conjugates. Second, the glucuronide structure of the bilirubin ester conjugates was verified by chromatographic analyses of ethyl anthranilate azoderivatives prepared from bile of transplanted animals, as described by Heirwegh and Blanckaert (11).

\section{Histology}

Spleens of recipient rats were fixed in $10 \%$ buffered formaldehyde and embedded in paraffin. Histological examination was performed on $6-\mu \mathrm{m}$ sections, stained with hematoxylin and eosin (H \& E) and by the periodic acid Schiff (PAS) staining procedure.

\section{Experimental design}

In the first series of experiments (series A) the effect of HTX on TB levels in the nonimmunosuppressed Gunn rat was studied, in both the allogenic and isogenic situation.

In the second series of experiments (series B) the mechanism of action of HTX was studied. Bile from immunosuppressed HTX-treated Gunn rats was examined for the presence of bilirubin glucuronides.

In the third series of experiments (series $\mathrm{C}$ ) bile analysis of individual recipient Gunn rats was performed at various time intervals after HTX, to study whether or not bilirubin conjugation was rejection related.

Series A. Six Gunn rats (group A-I) were transplanted with Wistar hepatocytes. A control group of 6 Gunn rats (group A-II) received Gunn hepatocytes. Six Wistar rats (group A-III) underwent isogenic HTX to serve as a second control group in which survival of hepatocytes transplanted in an isogenic situation was tested. In all groups blood samples $(0.5 \mathrm{ml})$ were obtained by paraorbital puncture on the day prior to HTX and twice a week starting the second day after HTX during 4 weeks. TB was measured in all samples. A final sample was obtained after 2-3 months, after which the animals were sacrificed by cervical dislocation. Spleens of all animals were examined histologically.

Series B. Thirty-two Gunn rats underwent total-body irradiation of 600 rad $\mathrm{X}$ rays 2 days prior to HTX. These rats were divided into four groups of 8 rats. Group B-I was transplanted with $10^{7}$ viable Wistar hepatocytes, and group B-II with $10^{7}$ nonviable Wistar hepatocytes (cells were killed by repeated freezing and thawing until cell viability was $0-1 \%$ ). Group B-III received $10^{7}$ viable Gunn hepatocytes. Group B-IV underwent a sham operation by intrasplenic injection of $1 \mathrm{ml} \mathrm{Krebs-Henseleit}$ solution.

On day 6 the common bile duct of all rats was cannulated with polyethylene tubing (PE-50) under Nembutal anesthesia, and the cannula was exteriorized at the neck. 


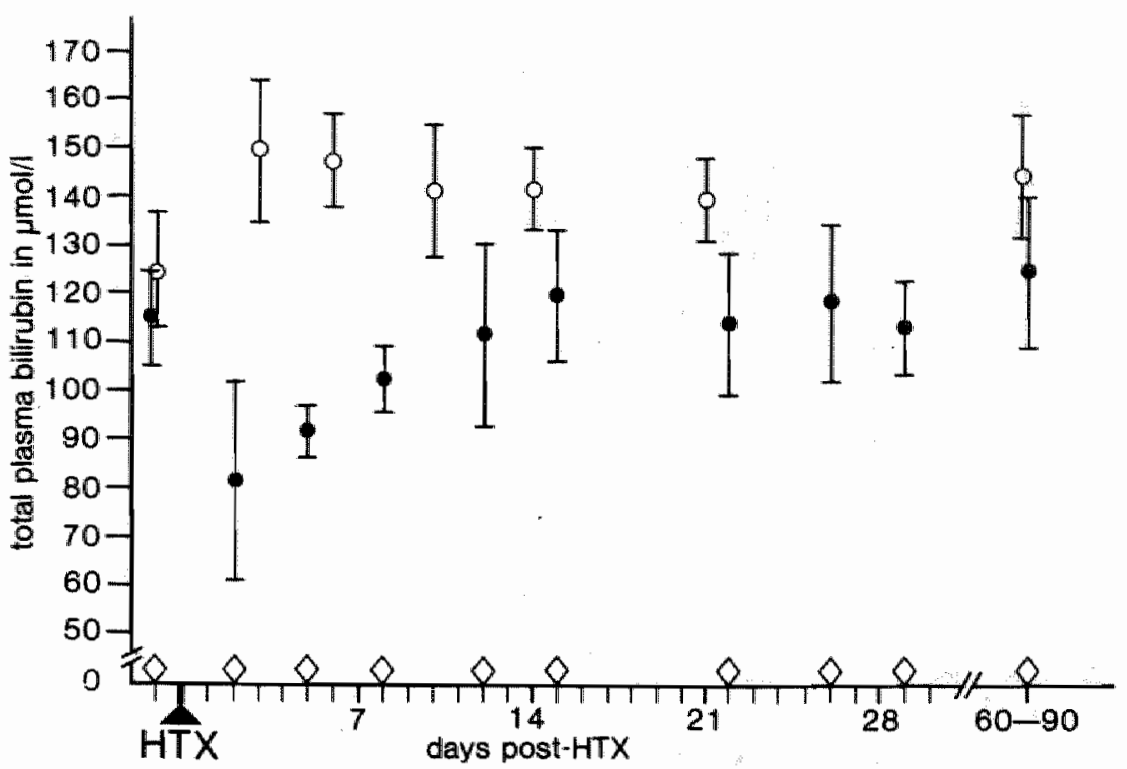

Figure 1. Transient decrease of total plasma bilinubin in the Gurn rat following transplantation with Wistar hepatocytes. Means and $S D$ are given.

- Group A-r: Gunn rats receiving Wistar hepatocytes; $n=6$.

- Group A-II: Gunn rats receiving Gunn hepatocytes:" $n=6$.

Group A-III: Wistar rats receiving Wistar hepatocytes; $n=6$.

Approximately $2 \mathrm{ml}$ of bile was collected under dim light into tubes placed in crushed ice, while the animals could move freely and eat and drink. All animals were sacrificed after bile sampling.

Series C. Eight Gunn rats received a permanent bile fistula between 3 and 2 weeks prior to HTX or sham HTX. Four of these Gunn rats underwent HTX with viable Wistar hepatocytes (Group C-I), and the other four were sham-treated (Group CII). Four Gunn rats without a permanent bile fistula were also sham-treated (Group C-III) to serve as a second control group. All animals remained in good physical condition with normal weight gain.

In all three groups TB levels were determined twice a week during the experiment starting 1 week before HTX. Bile sampling for chromatographic analysis was performed in Groups C-I and C-II just prior to HTX and on days 2, 6, 16 and 30 after HTX. All animals were sacrificed 1 month after HTX and the spleens were histologically examined.

Results

Series A

Effect of HTX on TB in the Gunn rat. The results for the 3 groups of rats in series 
A are shown in Figure 1. All Gunn rats that received viable Wistar hepatocytes (Group A-I) exhibited a decrease of TB. The mean TB level on days 2,5 and 8 following HTX was significantly lower $(\mathrm{p}<0.05)$ compared to the pre-HTX value. Thereafter the mean TB returned to the preoperative level, which was attained on day 12 , and remained at this level during the test period, which extended to 3 months after transplantation. During the period between day 2 and day 10 after HTX TB values observed in Group A-I also differed significantly $(p<0.02)$ from the corresponding TB values of Gunn rats receiving Gunn hepatocytes (Group A-II). In fact, the latter group exhibited a transient increase of TB following HTX. A similar increase was also observed in pilot studies in Gunn rats, subjected to extensive surgical trauma or infection (data not given). Group A-III, Wistar rats receiving Wistar hepatocytes, showed unchanged, i.e., unmeasurably low, TB levels pre- and postoperatively.

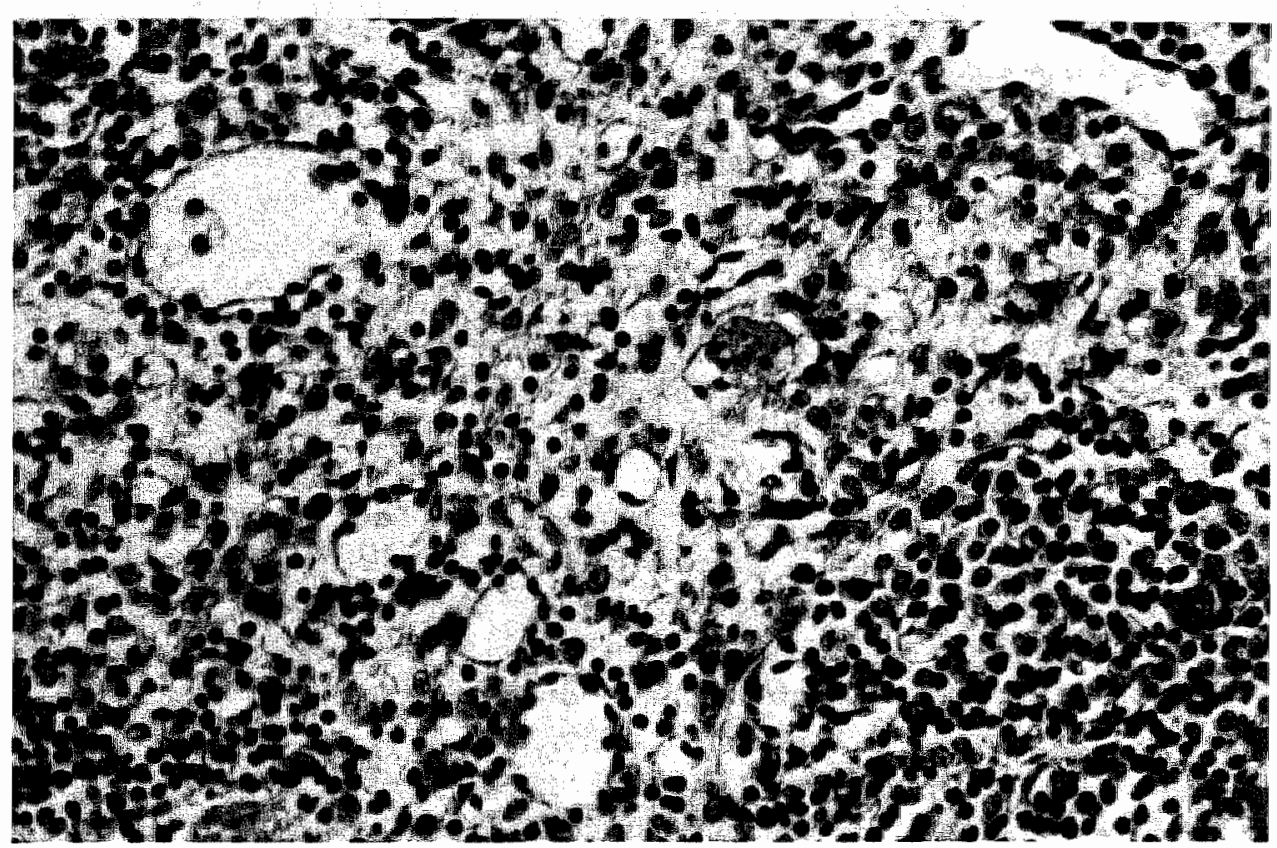

Figure 2. Wistar to Gunn allogenic hepatocyte rransplantation into the spleen (Group A-I). Microscopic examination after 2 months: Only PAS-positive granules are observed in the splenic red pulp. Morphologically intact hepatocytes are not found (PAS, x 100).

Histological observations. Histological examination of the spleen 2 to 3 months post-HTX of Gunn rats treated with Wistar hepatocytes (Group A-1) did not reveal liver cells in the spleen. PAS-positive granules included in leukocytes or in the vicinity of multinuclear giant cells were found in the red pulp. Possibly these granules were the remnants of rejected hepatocytes (Figure 2).

In the isogenic transplanted Groups A-II and A-III, the spleens of all animals con- 


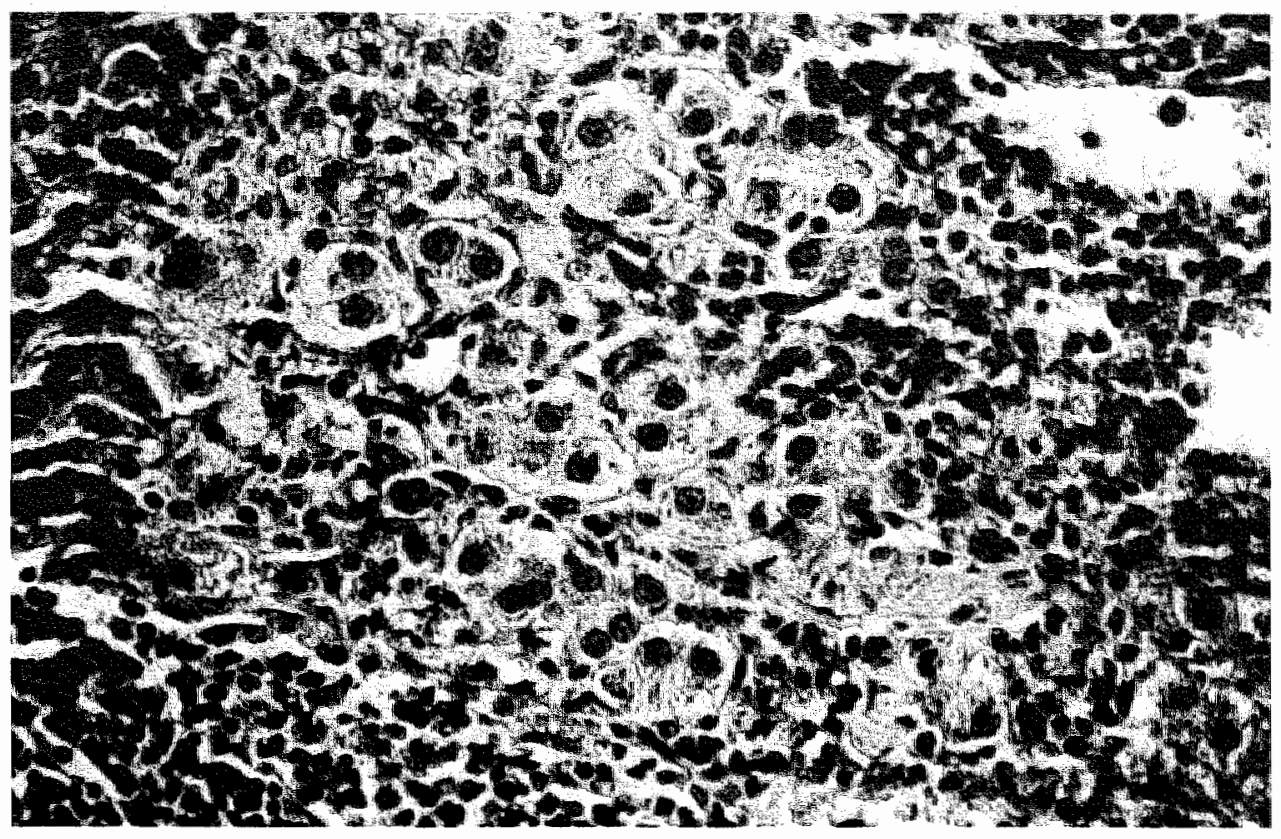

Figure 3. Wistar to Wistar isogenic hepatocyte transplantation into the spleen after 3 months (Group A-III). Aggregates of surviving hepatocytes are present in the red pulp of the recipient spleen.

Note the typical binuclear appearance (H\&E, $x 100)$.

tained cells morphologically identical with intact hepatocytes, containing PASpositive granules and often with typical binuclear appearance. Aggregates of hepatocytes were present, but without formation of hepatic cords as in normal liver tissue. These aggregates ranging from several cells to several hundreds of cells were scattered throughout the red pulp of the spleen (Figure 3). Similar histological findings were reported by Mito and co-workers $(12,13)$.

\section{Series B}

Structure analyses of the bilirubins in bile of rats following HTX. In vivo formation and biliary excretion of bilirubin esters were demonstrated in all Gunn rats transplanted with viable Wistar hepatocytes (Group B-I). Monoesterified and diesterified bilirubins were detected in all chromatograms from bile of Gunn rats of this group (Figure 4). Moreover, the glucuronide structure of these monoconjugated and diconjugated bilirubins was demonstrated.

By contrast, bilirubin monoglucuronide and bilirubin diglucuronide were not detectable in bile of Gunn rats transplanted with nonviable Wistar hepatocytes (Group B-II), which indicates that only viable Wistar hepatocytes can account for bilirubin 

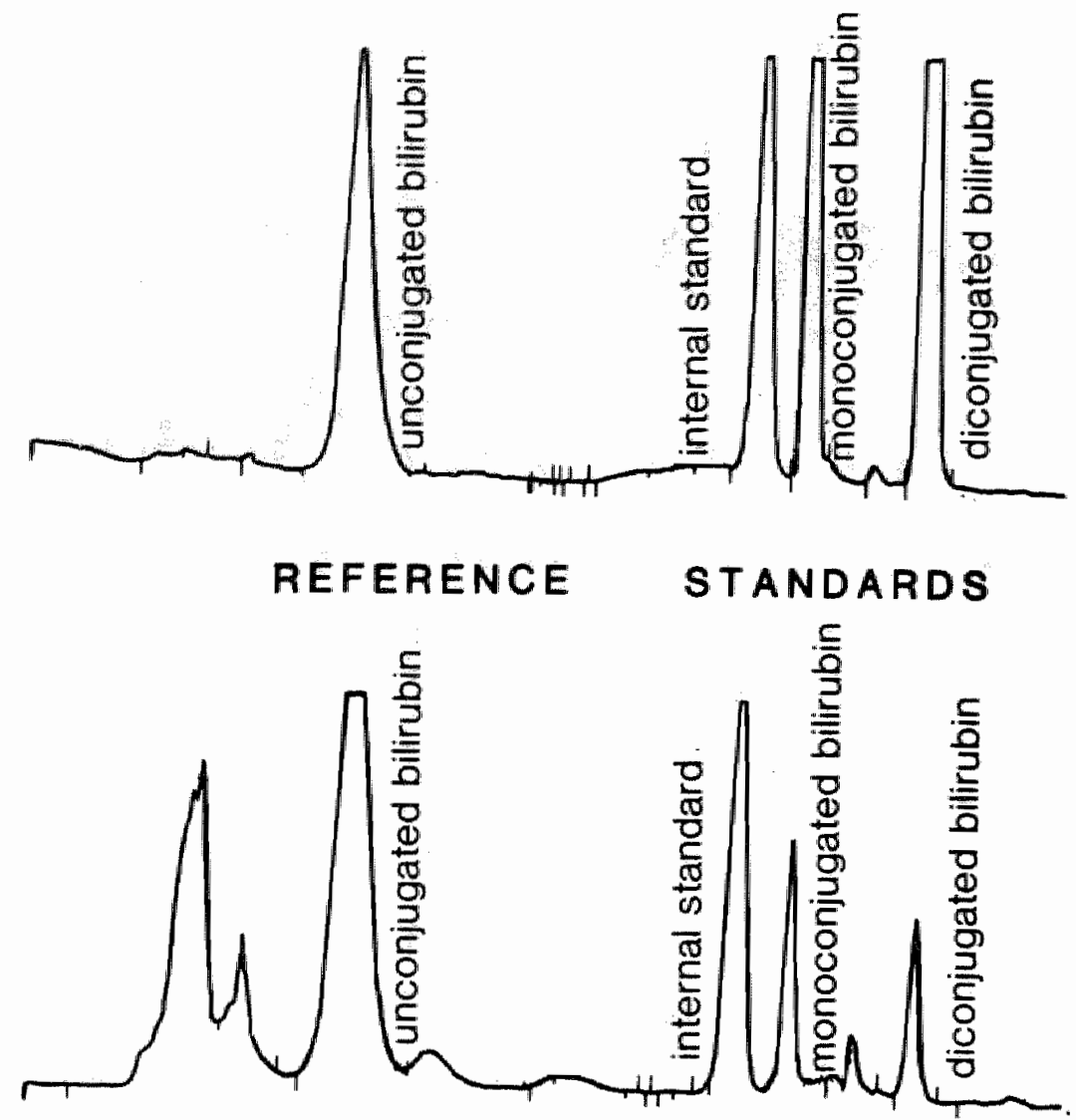

BILE OF HTX-TREATED GUNN RAT

Figure 4. The upper pattern represents a chromatogram of the reference compounds. The lower pattern is a chromatogram of bile from Gunn rats tranplanted with $10^{7}$ wiable Wistar hepatocytes. Note the peaks for mono- and diconjugated bilirubin. Peaks for mono-and diconjugated bilirubins are absent in chromatograms of bile from nontreated Gunn rats (not shown in the Figure).

glucuronidation. As expected bilirubin glucuronides were also absent in all Gunn rats receiving Gunn hepatocytes or a sham operation (control Groups B-III and B-IV, respectively). Chromatographic patterns in animals of these last three groups were the same as those in nontreated Gunn rats, in which only unconjugated bilirubin was detected. 
Effect of HTX on TB in the Gunn rat with a bile fistula. TB levels of Gunn rats receiving Wistar hepatocytes (Group C-I) decreased sharply after HTX (Figure 5). Mean TB was significantly lower after HTX than before $(p<0.05)$. In all animals TB levels started to rise after day 13 , yet the preoperative level was not attained at the end of the experiment. Interestingly, TB levels in the sham-treated Gunn rats with a bile fistula (Group C-II) gradually decreased during the experiment, but comparison of the TB levels of Groups C-I and C-II showed significantly lower values in Group C-I from day 2 to day 13 post-HTX $(p<0.05)$. From day 16 till the end of the experiment significant differences between these two groups were not observed. Group C-III did not show a statistically significant decrease in mean TB during the experiment. These data indicate that the bile fistula must have had an effect on TB levels, resulting in a decrease of TB in both Groups C-I and C-II. The sharp decrease after HTX observed in Group C-I however is explained by the effect of HTX with Wistar hepatocytes. This explanation is substantiated by the data from the bile analyses in this series.

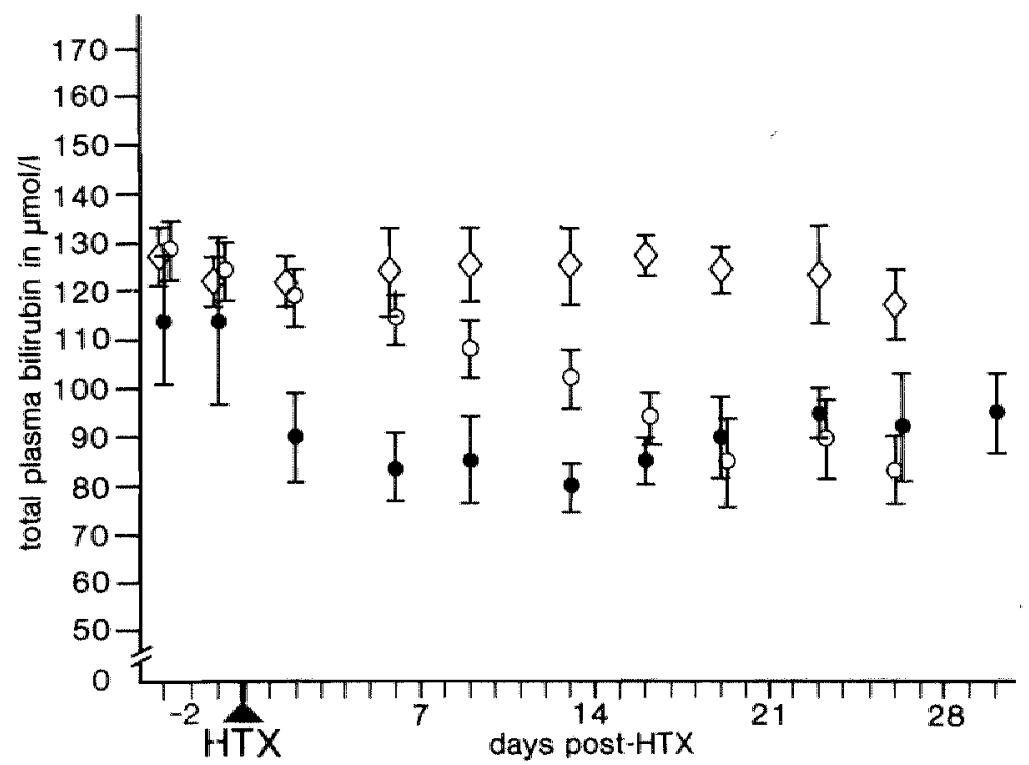

Figure 5. Total plasma bilirubin levels in the Gunn rat with a permanent bile fistula following transplantation with Wistar hepatocytes. Means and S.D. are given.

- Group C-I: Gunn rats with a bile fistula receiving Wistar hepatocytes; $n=4$.

- Group C-II: sham-treated Gunn rats with a bile fistwla: $n=4$.

$\checkmark$ Group C-III: sham-treated Gunn rats without a bile fistula; $n=4$.

Bile analyses following HTX. All bille specimens of the animals of Group C-I contained bilirubin glucuronides on day 2 and day 6 . Before HTX and from day 16 on- 
ward bilirubin glucuronides were not found in bile from these animals (Figure 6). The formation of bilirubin monoglucuronide was present on day 2 and on day 6 , although diminished on day 6 . The formation of bilirubin diglucuronide was only observed on day 2 .

On day 2 the relative amount of the bilirubin glucuronides (mono- plus diglucuronides) varied from 4.8 to $11.2 \%$ (mean $7.0 \%$ ) of the total amount of bilirubin excreted in bile. The mean biliary concentration of bilirubin glucuronides was $0.41 \mu \mathrm{M}$. Billirubin monoglucuronide ranged from 2.3 to $9.4 \%$ (mean $4.6 \%$ ) of the excreted total bilirubin, with a mean biliary concentration of $0.24 \mu \mathrm{M}$. Bilirubin diglucuronide varied from 1.8 to $3.4 \%$ (mean $2.4 \%$ ), with a mean biliary concentration of $0.17 \mu \mathrm{M}$ ).

On day 6 only the monoester was formed, varying between 0 and $3.6 \%$ of the excreted total bilirubin (mean $1.6 \%$ ) with a mean biliary concentration of $0.09 \mu \mathrm{M}$.

As expected, neither bilirubin mono-, nor diglucuronide were found in bile specimens of Group C-II.

Histological observations. Histology of the spleens of Group C-I revealed the same picture as in Group A-I. The spleens of Groups C-II and C-III had a normal histological appearance.

\section{Discussion}

Our first series of experiments showed that a transient decrease of TB in the nonimmunosuppressed Gunn rat was achieved by intrasplenic transplantation of nondeficient Wistar hepatocytes. In Gunn rats treated with viable Wistar hepatocytes, TB levels returned to preoperative levels after approximately 12 days, which is the period required for liver allograft rejection in rats across a weak histocompatibility barrier (14). Histological observations showed that rejection occurred in allogenic HTX, whereas in the isogenic situation grafted hepatocytes survived. These findings led to the assumption that survival of transplanted nondeficient hepatocytes was necessary for the lowering of TB levels, and that rejection of the transplanted hepatocytes abolished this effect. This was confirmed by the findings in series B and C.

Our hypothesis is that surviving transplanted hepatocytes maintain enzyme function until the time of rejection and account for the conjugation of bilirubin. After conjugation in the transplanted hepatocytes, bilirubin glucuronides are then excreted by the host rat liver into bile, since the Gunn rat is able to excrete previously conjugated bilirubin into bile (15). The possibility that microsomes or the enzyme bilirubin UDP-glucuronyl transferase itself continue to function after cytolysis of the transplanted cells is very unlikely, since transplantation of nondeficient, nonviable hepatocytes, damaged by a repeated freeze-thaw cycle, did not result in the appearance of the deficient enzyme activity. Other mechanisms, such as genetic transformation in the deficient liver through incorporation of informational macro- 


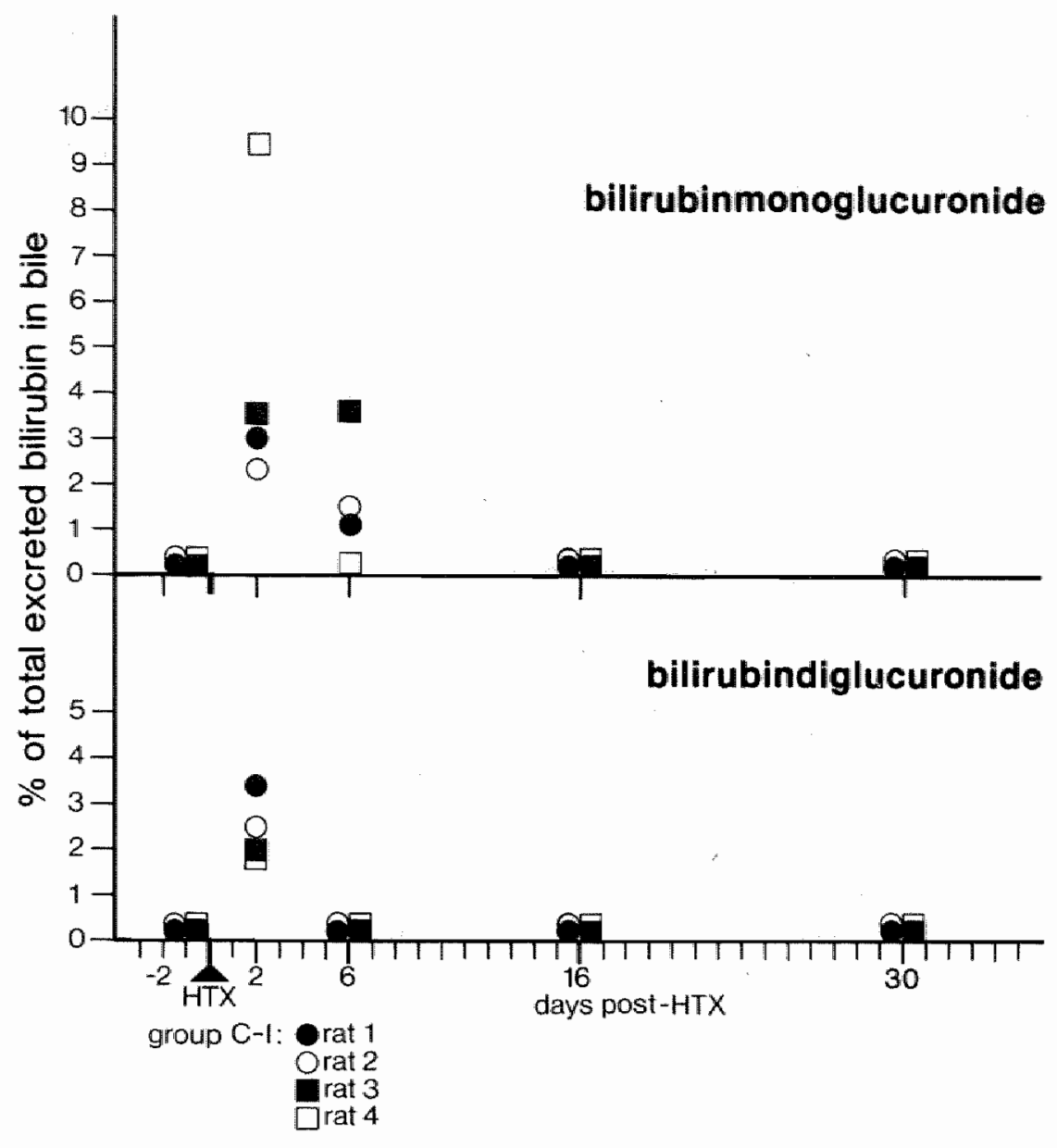

Figure 6. This figure shows the relative amounts of bilirubin mono- and diglucuronides in bile of individual Gunn rats at various intervals after HTX with wiable Wistar hepatocytes (Group C-1). Bile from sham-treated Gunn rats (Group C-II) did not contain BMG or BDG at any interval after HTX (not shown in the Figure).

molecules from transplanted liver cells or the production of a diffusible derepressor substance by the nondeficient liver cells, opening up the inactive genetic locus for bilirubin UDP-glucuronyl transferase in the Gunn rat hepatocytes, have been proposed (16). Such molecules could theoretically be released from non-heatdestructed normal hepatocytes. Our second series of experiments, however, showed that bilirubin conjugation was not observed after transplantation of hepatocytes killed by a freeze-thaw cycle and renders the latter mechanisms unlikely. Sutherland et al. (17) and Makowka et al. $(18,19)$ applied HTX in the treatment of hepatotoxin-induced acute hepatic failure in rats, and observed a considerable im- 
provement in survival. Makowka (19) demonstrated that a heat-stable factor, present in the cytosol of subcellular fractions of hepatocytes stimulated the rate of endogenous regeneration of the injured liver, and was responsible for the increase in survival observed. The positive effect of HTX in the treatment of enzyme deficiency disease and of acute hepatic failure thus appears to be based on two different mechanisms of action: for the appearance of the deficient enzyme activity the presence of transplanted viable, nondeficient hepatocytes are required, whereas for the treatment of acute hepatic failure regeneration of the affected liver is enhanced by a heat-stable factor, that can be isolated from hepatocytes.

Rugstad et al. (20) demonstrated in 1970 that subcutaneously transplanted rat hepatoma $\left(\mathrm{MH}_{1} \mathrm{C}_{1}\right)$ cells were able to bring about bilirubin conjugation in the Gunn rat. Our studies demonstrate that transplantation of nonneoplastic hepatocytes can produce an identical effect.

In summary, it is concluded that allogenic transplanted hepatocytes undergo rejection without immunosuppressive therapy. Allogenic HTX with Wistar hepatocytes in nonimmunosuppressed Gunn rats causes a transient decrease of TB. This decrease of $\mathrm{TB}$ is explained by the appearance of bilirubin glucuronidation and subsequent excretion of bilirubin glucuronides in the bile of the recipient Gunn rat. Surviving and functioning hepatocytes from a nondeficient donor are required for the appearance of bilirubin UDP-glucuronyl transferase activity.

HTX has the potential of curing enzyme deficiencies, if nondeficient hepatocytes would not be rejected by the deficient host, and transplanted hepatocytes proliferate as is stated by Kusano (21). A new promising approach is autotransplantation of enzyme-deficient cells, after recombinant DNA technology has provided the correct genetic information for the production of the deficient enzyme (22). This may render HTX applicable for clinical use in the future.

\section{Acknowledgements}

The authors thank Dr. C.J. van der Linden (Maastricht) for discussing the manuscript, Mrs. K. Spronck (Maastricht) for her help with the preparation of the manuscript and Mrs. N. Rijskamp (Maastricht), Mr. R. Remy (Groningen), Mr. P. Willems (Maastricht), Mr. G. Michiels (Leuven) and Mr. J. van Dongen (Maastricht) for technical assistance. Mr. C. Voskamp (Maastricht) prepared the figures.

This paper has been published in the Journal of Surgical Research. Vroemen JPAM, Blanckaert N, Buurman WA, Heirwegh KPM, Kootstra G. Treatment of enzyme deficiency by hepatocyte transplantation in rats. Journal of Surgical Research 1985; 39: 267. 


\section{References}

1. Desnick RJ, Simmons RL, Allen KY.

Correction of enzymatic deficiencies by renal transplantation: Fabry's disease.

Surgery 1972; 72: 203.

2. Brady RO, Pentchev $P G$, Gal AE, Hibbert SR, Dekaban AS.

Replacement therapy for inherited enzyme deficiency. Use of purified glucocerebrosidase in Gaucher's disease.

N Eng』 $\mathbb{I}$ Med 1974; 291: 989.

3. Hood JM, Koep LJ, Peters RL at al.

Liver transplantation for advanced liver disease with alpha-1 wantitrypsine deficiency.

N Engl J Med 1980; 302: 272.

4. Matas AJ, Sutherland DER, Steffes MW et al.

Hepatocellular transplantation for metabolic deficiencies: decrease of plasma bilirubin in Gunn rats.

Science 1976; 192: 892 .

5. Groth CG, Arborgh B, Björkén C, Sundberg G, Lundgren G.

Correction of hyperbilirubinemia in the glucuronyl transferasedeficient rat by intraportal hepatocyte transplantation.

Transplant Proc 1977; 9: 313.

6. Sutherland DER, Matas AJ, Steffes MW, Simmons RL, Najarian IS.

Transplantation of liver cells in an animal model of congenital enzyme deficiency: the Gunn rat. Transplant Proc 1977; 9: 317.

7. Gunn $\mathrm{CH}$.

Hereditary acholuric jaundice in a new mutant strain of rats.

J Hered 1938; 29: 137.

8. Seglen PO.

Preparation of isolated rat liver cells.

Methods Cell Biol 1976; 13: 29.

9. Vonck RJ, Van Doorn ABD, Strubbe JH.

Bile secretion and bile composition in the freely moving, unanaesthetized rat with a permanent biliary drainage: Influence of food intake on bile flow.

Clin Sci Mol Med 1978; 55: 253.

10. Blanckaert N, Kabra PM, Farina FA, Stafford BE, Marton $L J$, Schmid $\mathbb{R}$.

Measurement of bilirubin and its monoconjugates and diconjugates in human serum by alkaline methanolysis and high performance liquid chromatography.

J Lab Clin Med 1980; 96: 198.

11. Heirwegh KPM., Blanckaert $\mathbb{N}$.

Analysis of bilirubin conjugates.

Methods Enzymol 1981; 77: 391.

12. Mito M, Ebata H, Kusano M, Onishi T, Hiratsaka M, Saito T,

Studies on ectopic liver utilizing hepatacyte transplantation into the rat spleen.

Transplant Proc 1979; 11: 585.

13. Mito M, Ebata H, Kusano M, Onishi T, Saito T, Sakamoto S.

Morphology and function of isolated hepatocytes transplanted into rat spleen.

Transplantation $1979 ; 28: 499$.

14. Lee $S$, Edgington TS.

Heterologic liver transplantation utilizing inbred rat strains. I. Characterization of allogeneic graft rejection and the effects of biliary obstruction and portal vein circulation on liver regeneration. Amer J Pathol 1968; 52: 649.

15. Arias LM, Johnson $L$, Wolfson $S$.

Biliary excretion of injected conjugated and unconjugated bilirubin by normal and Gunn rats.

Amer J Physiol 1961; 200: 1091.

16. Mukherjee AB, Krasner J.

Induction of an enzyme in genetically deficient rats after grafting of normal liver.

Science (Washington DC) 1973; 182: 68 . 
17. Suthertand DER, Numata M Matas AJ, Simmons RL, Najarian JS. Hepatocellular transplantation in acute liver failure.

Surgery 1977; 82: 124 .

18. Makowka L, Rotstein LE, Falk RE et al.

Alogeneic and xenogeneic hepatocyte transplantation in experimental hepatic failure.

Transplantation $1980 ; 30: 429$.

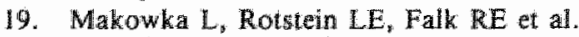

Studies into the mecharism of reversal of experimental acute hepatic fallure by hepatocyte trans. plantation.

Canad I Surg 1981; 24: 39.

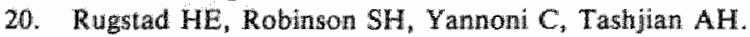

Transfer of bilirubin uridine diphosphate-glucuronyl transferase to enzyme-deficient rats. Scierice 1970; 170: 553 .

21. Kusano M, Mito M.

Observations on the fine structure of longsurvived isolated hepatocytes inoculated into rat spleen.

Gastroenterology 1982; 82: 616 .

22. Brennand I, Konecki DS, Cáskey Cr.

Expression of human and chinese hamster hypoxanthine-guanine phosphoribosyltransferase cDNA recombinants in cultured Lesh-Nyhan and Chinese hamster fibroblasts.

$J$ Biol Chem 1983; 258: 9593 . 
The congenic normal R/APfd and jaundiced $R / A P f d-j / j$ rat strains: a new animal model of hereditary nonhemolytic unconjugated hyperbilirubinemia due to defective bilirubin conjugation

\section{Summary}

In this paper the production of the $R / A P f d-j / j$ strain which is congenic with the $\mathrm{R} / \mathrm{APfd}$ strain is reported. The $\mathrm{R} / \mathrm{APfd}-\mathrm{j} / \mathrm{j}$ completely lacks hepatic bilirubin UDPglucuronyl transferase activity, as do our GUNNXR/Pfd-j/j rat strain and various other stocks of Gunn rats $(\mathrm{j} / \mathrm{j})$ described in the literature. Our recombinant inbred strain GUNNXR/Pfd-j/j was produced from non-inbred Gunn $(j / j)$ rats. This GUNNXR/Pfd-j/j rat was used as a donor of the jaundice gene $\mathrm{j}$, the R/APfd rat serving as the recipient. After 8 backcross-intercross cycli (16 generations) the $\mathrm{R} / \mathrm{APfd}-\mathrm{j} / \mathrm{j}$ strain was obtained which is congenic with the R/APfd strain. Congenicity was demonstrated by various techniques including transplantation of skin tissue, strain-specific tumour cells and hepatocytes, the mixed lymphocyte reaction, and comparison of biochemical markers. The potential of the novel inbred strain of jaundiced rat, $\mathrm{R} / \mathrm{APfd}-\mathrm{j} / \mathrm{j}$, and the corresponding control strain R/APfd for biochemical studies of bilirubin metabolism are briefly discussed. 


\section{Introduction}

In 1938, Gunn described a recessive mutant rat strain (1) which represents an animal model of human hereditary jaundice known as the Crigler-Najjar syndrome (2) characterized by a total deficiency of bilirubin UDP-glucuronyl transferase activity (for reviews of this model see: Gunn (3), Robinson (4), Swarm (5), Calabrese (6) and Cornelius (7)). The original Gunn rat arose as a recessive mutation in a colony of Wistar rats (1). The mutation which causes defective conjugation of bilirubin has been denoted by the symbol ' $\mathrm{j}$ ', derived from jaundice. Inheritance of the trait follows the laws of Mendel. Because of low fertility $(8,9,10)$ Gunn rats have frequently been crossed with rats of other strains and stocks to increase the capacity of reproduction. At present, non-inbred, inbred and recombinant inbred strains of the Gunn rat exist $(11,12,13)$. The only generally valid statement about these Gunn rats is that they are all deficient in the enzyme bilirubin UDP-glucuronyl transferase (EC 2.4.1.17).

For experimental studies, especially with regard to bilirubin metabolism and organ transplantation, a suitable control strain for the jaundiced rat would be of great value. In the present paper, the method used for obtaining the congenic strain $R / A P f d-j / j$ is described. The recessive, monogenetic, autosomal factor $j$ of the GUNNXR/Pfd- $\mathrm{j} / \mathrm{j}$ rat was transferred to the inbred R/APfd rat by 8 backcrossintercross cycli, yielding the $\mathrm{R} / \mathrm{APfd}-\mathrm{j} / \mathrm{j}$ strain which is congenic with the $\mathrm{R} / \mathrm{APfd}$ strain. Evidence indicating that the $R / A P f d-j / j$ and $R / A P f d$ strains are congenic and that the $\mathrm{R} / \mathrm{APfd}-\mathrm{j} / \mathrm{j}$ rat has a total defect of bilirubin conjugation is given.

\section{Materials and methods}

\section{Development of the $\mathbf{R} / \mathbf{A P f d}-\mathbf{j} / \mathbf{j}$ strain.}

In order to obtain the congenic $R / A P f d-j / j$ strain the jaundice gene of the GUNNXR/Pfd-j/j strain was transferred to the inbred R/APfd strain by 8 backcross-intercross cycli.

The R/APfd strain. In 1947, Prof. Muhlbock, Antoni van Leeuwenhoekhuis, Dutch Cancer Institute, Amsterdam, The Netherlands, derived the inbred R/A strain from a WIST stock obtained in 1947 from the Wistar Institute. We obtained a breeding colony of R/A rats in 1961 and continued the breeding by brother $\mathrm{x}$ sister crossing. After 20 generations of inbreeding in the Proefdierencentrum (Laboratory Animal Center) in Leuven, the inbred strain has been denoted by the code R/APfd.

Origin of the GUNNXR/Pfd-j/j strain. After its discovery in 1934 (1) the Gunn rat strain was transferred to the Department of Genetics, University of California, Berkeley, CA, USA, where Gunn rats $(\mathrm{j} / \mathrm{j})$ were reared at random from heterozygotes 


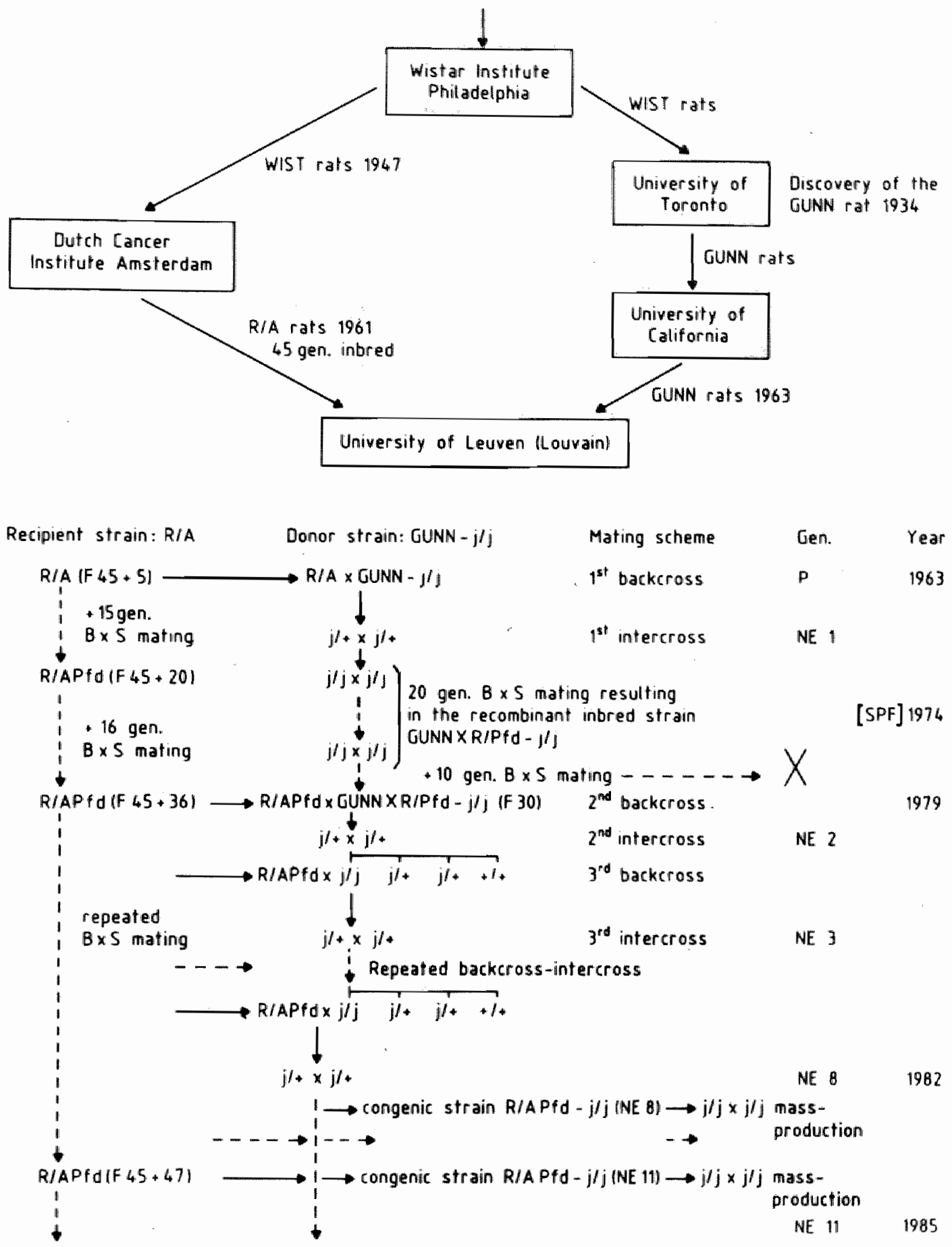

Figure 1. History and development of the congenic $R / A P f d-j / j$ rat strain. 
$(j /+x j /+)_{s}$ with application of an outcross when fertility declined. We obtained two couples of hooded Gunn rats from the University of California in 1963. These animals served as starting material for our breeding experiments. The GUNNXR/Pfd-j/j strain was obtained by one outcross with the inbred R/APfd strain (to increase fertility) followed by exclusive brother $x$ sister inbreeding. Singleline breeding was adopted to render the relationship as close as possible. From the 20 th generation of brother $x$ sister inbreeding onwards, the strain could be considered as a recombinant inbred strain. The strain is syngenic and thus also histocompatible, and is called the GUNNXR/Pfd- $j / j$ strain. The biochemical experiments described in the present paper were performed with rats belonging to the 40th inbred generation of the GUNNXR/Pfd-j/j strain.

Establishment of the congenic R/APfd-j/j strain. In 1974 the R/APfd and GUNNXR/Pfd-j/j strains were rendered specified pathogen free by standard procedures (sterile hysterectomy, foster nursing and barrier maintenance). The first backcross of the GUNNXR/Pfd-j/j strain with R/APfd strain was performed in 1979, at which time the 30th inbred generation of the GUNNXR/Pfd $-\mathrm{j} / \mathrm{j}$ strain and the 81st generation of the R/APfd strain had been reached. From 1979 onwards backcrosses to the R/APfd strain were performed systematically to produce the R/APfd$\mathrm{j} / \mathrm{j}$ strain possessing the genetic material of the R/APfd rat with retainment of the gene coding for the enzymatic defect. Each backcross was followed by an intercross $(j /+x j /+)$ to enable sellection of the $j$ trait, since the jaundice gene $j$ is only expressed phenotypically in homozygous $\mathrm{j} / \mathrm{j}$ animals by their yellow coat. The history and the backcross-intercross scheme are given in Figure 1. After the 8th cycle a breeding colony for mass production was started from parents derived from the backcross colony. The offspring of the breeding pairs of the mass-production colony were not used for further breeding. Instead, to maintain single-line breeding and to improve congenicity further, new breeding pairs were taken from subsequent stages of the backcross-intercross line. This colony is referred to as the R/APfd-j/j strain. The growth and reproduction characteristics of the colony were compared with those of the R/APfd strain.

Breeding environment. Relevant factors of the breeding conditions are as follows: temperature, $22.5^{\circ} \mathrm{C} \pm 2.5^{\circ} \mathrm{C}$; relative humidity, $40-80 \%$; ventilation, the air is completely replaced by fresh filtered air every $3 \mathrm{~min}$, (recirculation is not used); hyperbaric pressure, $15 \mathrm{~mm}$ water column (150 $\mathrm{Pa}$ ) (in 3 steps); photoperiodicity, illumination for $14 \mathrm{hr}$, darkness for $10 \mathrm{hr}$; intensity of light, $400 \mathrm{~lx}$ at $1 \mathrm{~m}$ under the ceiling in the breeding rooms, considerably less in the cages; noice level, less than $60 \mathrm{~dB}$; cages, Makrolon type III and stainless steel, $58 \times 20 \times 20 \mathrm{~cm}$; food, pelleted $\gamma$ irradiated (1 Mrad) GS food from Hope Farms, ad libitum; drinking water, water acidified with $\mathrm{HCl}$ to $\mathrm{pH} 2.8$, in water bottles, ad libitum. 
Congenicity between the R/APfd and R/APfd-j/j strains was verified by histocompatibility testing and by comparison of biochemical markers. Histocompatibility testing involved transplantation of skin, tumour cells, and liver cells, as well as mixed lymphocyte reactions (MLRs).

Skin transplantation. The method described by Waynforth (14) for grafting tail skin to the back was used. This technique facilitates the interpretation of the occurrence or non-occurence of rejection.

Transplantation of tumour cells. The transplantable mouse sarcoma virus $\mathrm{F} 3 / \mathrm{I}$ tumor, induced in R/APfd rats and specific for this rat strain (15), was used. Acceptor $R / A P f d(n=9)$ and $R / A P f d-j / j$ rats $(n=9) 5$ weeks old were pre-immunized by intraperitoneal injection of lymphocytes obtained from the thymus, spleen and lymph nodes of R/APfd donor rats. Nine days later, $5 \times 10^{5} \mathrm{~F} 3 / \mathrm{I}$ tumour cells in a volume of $0.2 \mathrm{ml}$ were injected subcutaneously in the right abdominal wall of the acceptor rats. Tumour development was monitored by palpation at days 4, 6, 11 and 24 after inoculation and by histological examination at autopsy (day 27). Tumour development in both rat strains was compared.

Hepatocyte transplantation. A two-step collagenase digestive method, modified after Seglen (16), was used for the isolation of rat hepatocytes. This isolation method resulted in an average yield of (2-4) $\times 10^{8}$ hepatocytes per rat liver, with a viability of $85-95 \%$ (trypan blue exclusion test). Hepatocyte transplantation was performed by slow injection of $1 \mathrm{ml}$ of the hepatocyte suspension, containing $10^{7}$ viable cells, into the spleen of the recipient rat. Male R/APfd and R/APfd-j/j rats were used as hepatocyte donors and recipients. Three generations of the $R / A P f d-j / j$ strain were involved in three consecutive series of hepatocyte transplantation. Graft survival was studied at autopsy of the recipient rat by histological examination of the spleen $(6 \mu \mathrm{m}$ routine section, hematoxylin and eosin and periodic acid Schiff stains).

Mixed lymphocyte reaction. Lymphocytes were prepared from rat spleens followed standard techniques. Two-directional MLR experiments were performed in microtitre plates with $0.2 \mathrm{ml} /$ well, containing $10^{5}$ cells per well from each responder. The cells were cultured in RPMI 1640 (Sibco, Paisley, UK) containing $15 \%$ (vol./vol.) fetal calf serum, $5 \times 10^{-5} \mathrm{M} \beta$-mercaptoethanol and antibiotics under standard conditions. Lymphocyte stimulation was assessed as ${ }^{3} \mathrm{H}$-thymidine uptake on day 6 of culture. In each series, responsiveness was tested between two $\mathrm{R} / \mathrm{APfd}-\mathrm{j} / \mathrm{j}\left(\mathrm{RT} 1^{\text {u }}\right)$ rats of the same generation, a $\mathrm{R} / \mathrm{APfd}\left(\mathrm{RT1} 1^{\text {u) }}\right)$ rat and a $\mathrm{BN}\left(\mathrm{RT}^{\mathrm{n}}\right)$ rat (positive control). In series $1, \mathrm{R} / \mathrm{APfd}-\mathrm{j} / \mathrm{j}$ rats from the 9 th backcross-intercross cycle were used; in series 2, R/APfd rats from the 10 th cycle 
were used. Each experiment was performed in triplicate and the mean value of ${ }^{3} \mathrm{H}$-thymidine uptake (expressed in counts per minute) was calculated.

Biochemical markers. The following biochemical markers were assayed in R/APfd and $R / A P f d-j / j$ rats according to the method of Bender et al. (17):

Acon-1, Adh-2, Alp-1, Es-1, Es-2, Es-3, Es-4, Es-6, Es-7, Es-9, Es-10, Es-13, Fh, Gdc, Gox, Hbb, Lap-1, Mgd, Mup-1, Pep-3, Pgd and Svp-1. The assays were kindly performed by Prof. L.F.M. van Zutphen (Department of Laboratory Animal Science, Faculty of Veterinary Medicine, University of Utrecht, Utrecht, The Netherlands).

\section{Assessment of bilirubin conjugation in the $\mathbf{R} / \mathbf{A P f d}-\mathbf{j} / \mathbf{j}$ strain}

Analyses were performed on male and female rats from the strains R/APfd, GUNNXR/Pfd-j/j and R/APfd-j/j (mean age, $100 \pm 3$ days). Blood $(1 \mathrm{ml}$ ) was collected under light ether anesthesia from a lateral tail vein. Serum was stored at $-20^{\circ} \mathrm{C}$ until analysis. For bile sampling, rats were anesthetized by intraperitoneal injection of $\mathrm{Nembutal}^{\mathrm{R}}(60 \mathrm{mg} / \mathrm{kg} \mathrm{b.wt}$.) and were provided with an external bile fistula. Bile was collected over four consecutive 1 -hr periods in tubes kept in the dark at $0^{\circ} \mathrm{C}$ and bile samples were pooled per individual rat for specific detection of billirubin conjugates. The animals were sacrificed by decapitation and the liver was quickly excised. Small liver fragments were rinsed in ice-cold $0.25 \mathrm{M}$ sucrose solution to remove adhering blood and were stored frozen at $-20^{\circ} \mathrm{C}$ until assayed for bilirubin UDP-glucuronyl tranferase activity.

Duplicate samples of serum $(50 \mu \mathrm{l})$ were used for determination of total bilirubin according to the diazo method of Nowros et al. (18). For each rat two $600 \mu \mathrm{l}$ samples of bile (one test and one blank sample) were stored at $-20^{\circ} \mathrm{C}$ for subsequent analysis by the alkaline methanolysis high pressure liquid chromatography (HPLC) procedure of Muraca \& Blanckaert (19). Using this procedure, the pattern and concentrations of bilirubins in serum were also determined for 5 female R/APfd-j/j rats and 5 female $\mathbb{R} / A P f d$ rats. The activity of bilirubin UDP-glucuronyl transferase was assayed on digitonin-activated liver homogenates $(25 \%$, wt./vol.) at incubation times of 10 and $20 \mathrm{~min}(20)$.

\section{Results}

\section{Growth and reproduction characteristics}

Weight curves are shown in Figure 2. In both strains, males show a significantly greater weight gain than females (both $\mathrm{p}<0.001$, $\mathrm{t}$-test). From week 2 onwards, male $\mathrm{R} / \mathrm{APfd}$ rats demonstrated a significantly greater weight gain than male $\mathrm{R} / \mathrm{APfd}-\mathrm{j} / \mathrm{j}$ rats $(p<0.01, t$-test) and this was also valid for female $R / A P f d$ versus female 


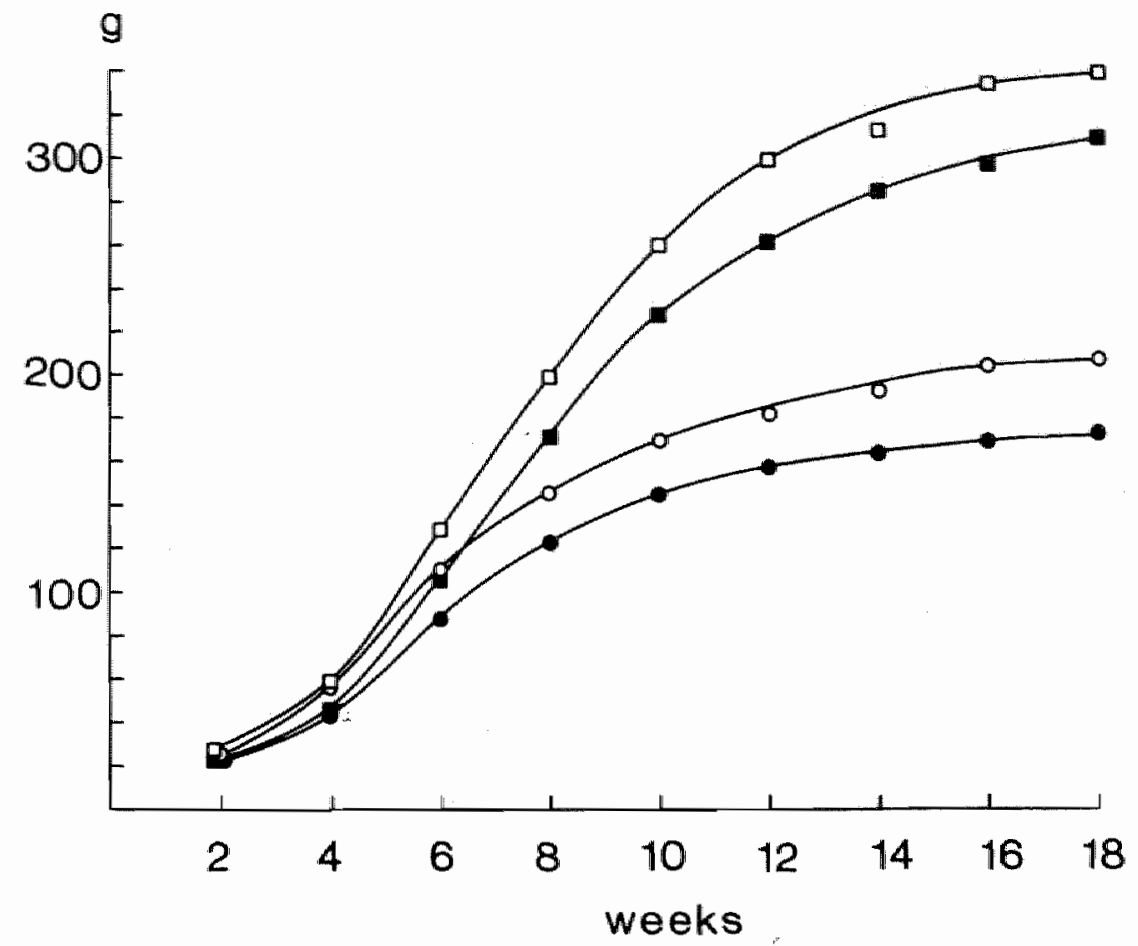

Figure 2. Weight curves of the R/APfd (open symbols, $\circ, \square$ ) and the R/APfd-j/j (closed symbols, •, congenic rat strains. Squares for males, circles for females.

$R / A P f d-j / j$ rats $(p<0.02$, t-test). The reproductivity coefficient (number of young weaned $/$ female/week) tested on 50 pairs of each of the R/APfd and R/APfd-j/j strains was equal to 0.86 and 1.0 , respectively, but this difference was not significant ( $p>0.05$, Mann-Whitney U-test).

\section{Histocompatibility tests}

Skin transplantation. Tail skin grafts within the R/APfd and $\mathrm{R} / \mathrm{APfd}-\mathrm{j} / \mathrm{j}$ strains survived for at least 120 days, demonstrating histocompatibility for both strains of rat. Skin transplants from $R / A P f d$ to $R / A P f d-j / j$ rats and vice versa took equally well, thus proving congenicity. Immunocompetence of the $R / A P f d-j / j$ rats was tested accross a major histocompatibility barrier by skin transplantation from allogenic donor $\mathrm{BN} / \mathrm{MaiPfd}$ rats. Immunogenicity of $\mathrm{R} / \mathrm{APfd}$ tissue was verified by skin transplantation to the BN/MaiPfd rats. As expected, allogenic tissue was rejected. The results are summarized in Table 1.

Transplantation of tumour cells. Tumour development was identical in the R/APfd and R/APfd-j/j strains. Furthermore, tumours removed at autopsy showed identical macroscopic and histological features in both strains. 
Table 1. Results of skin Iransplantation in virgin female rats

\begin{tabular}{llll}
\hline Donior strain & Host strain & $\begin{array}{l}\text { Number of } \\
\text { animalls }\end{array}$ & Graft survival \\
\hline R/APfd-j/j & R/APfd-j/j & 5 & all $>120$ days \\
R/APfd & R/APfd & 4 & all $>120$ days \\
R/APfd & R/APfd $-j / j$ & 6 & all $>120$ days \\
R/APfd-j/j & R/APfd & 6 & all $>120$ days \\
BN/MaPfd & R/APfd-j/j & 8 & 1,$16 ; 6,17 ; 1,21$ days \\
R/APfd-j/j & BN/MaPfd & 6 & 3,$15 ; 3,16$ days \\
\hline
\end{tabular}

Table 2. Hepatocyte transplantation between the R/APfd and $R / A P f d-j / j$ strains.

\begin{tabular}{|c|c|c|c|c|}
\hline Series & Donor & Recipient & $\mathrm{n}$ & Graft surwiwal \\
\hline \multirow[t]{2}{*}{ A } & $\mathrm{R} / \mathrm{APfd}$ & $\mathrm{R} / \mathrm{APfd} \times \mathrm{j} / \mathrm{j}$ & 2 & all $>180$ days \\
\hline & $\mathrm{R} / \mathrm{APfd}-\mathrm{j} / \mathrm{j}$ & R/APfd & 2 & all $>180$ days \\
\hline \multirow[t]{3}{*}{$\mathbf{B}$} & $\mathrm{R} / \mathrm{APfd}$ & $\mathrm{R} / \mathrm{APId}-\mathrm{j} / \mathrm{j}$ & 9 & all $>180$ days \\
\hline & $\mathrm{R} / \mathrm{APfd}-\mathrm{j} / \mathrm{j}$ & R/APfd & 6 & all $>180$ days \\
\hline & $\mathrm{R} / \mathrm{AP} / \mathrm{fd}-\mathrm{j} / \mathrm{j}$ & $\mathrm{R} / \mathrm{APfd}^{-j} / \mathrm{j}$ & 4 & all $>180$ days \\
\hline \multirow[t]{3}{*}{$\mathrm{C}$} & $\mathrm{R} / \mathrm{APfd}$ & $\mathrm{R} / \mathrm{APfd}-\mathrm{j} / \mathrm{j}$ & 8 & all $>180$ days \\
\hline & $\mathrm{R} / \mathrm{AP} / \mathrm{d}-\mathrm{j} / \mathrm{j}$ & R/APfd & 3 & all $>180$ days \\
\hline & $\mathrm{R} / \mathrm{APfd}-\mathrm{j} / \mathrm{j}$ & R/APfd-j/j & 8 & all $>180$ days \\
\hline
\end{tabular}

R/APfd-j/j rats from the 8 th (series A), 9 th (series B) and 10 th (series C) cycle were tested.

Table 3. Bidirectional mixed lymphocyte cultures (two series) between two R/APfd-j/f rats of the same generation (of the 9th and loth backcross-intercross cycle respectively), an $R / A P f d$ (RTIW) rat and a $B N$ (RTI $\left.{ }^{n}\right)$ rat.

\begin{tabular}{|c|c|c|c|c|c|}
\hline $\begin{array}{l}\text { MLC } \\
\text { series }\end{array}$ & & $R / A P f d-j / j$ & $R / A P f d-j / j$ & R/APfd & $\mathrm{BN}$ \\
\hline \multirow[t]{4}{*}{1} & R/APfd-j/j(NE9) & 1,884 & & & \\
\hline & R/APfd-j/j(NE9) & 1,990 & 1,874 & & \\
\hline & $\mathrm{R} / \mathrm{APfd}$ & 4,734 & 1,795 & 2,642 & \\
\hline & $\mathrm{BN}\left(\mathrm{RT} \mathrm{I}^{\mathrm{tr}}\right)$ & 15,673 & 17,074 & 15,410 & 2,991 \\
\hline \multirow[t]{4}{*}{2} & R/APfd-j/j(NE10) & 3,307 & & & \\
\hline & $\mathrm{R} / \mathrm{APfd} \mathrm{j} / \mathrm{j}(\mathrm{NE} 10)$ & 3,897 & 3,576 & & \\
\hline & R/APfd & $1,5,41$ & 3,132 & 3,274 & \\
\hline & $\mathrm{BN}\left(\mathrm{RT}^{\mathrm{n}}\right)$ & 26,552 & 20,916 & 24,433 & 5,641 \\
\hline
\end{tabular}

Lymphocyte stimulation per mixed lymphocyte culture is assessed as ${ }^{3} \mathrm{H}$-thymidine uptake in counts per minute on day 6 of culture. The listed values are the means of three mixed lymphocyte cultures.

Hepatocyte transplantation. The results of three series of transplantation experiments are shown in Table 2. Syngenically transplanted hepatocytes are known to survive in the recipient rat spleen without loss of metabolic functions or histologica] features (21). Hepatocyte transplantation between rat strains that are disparate in major histocompatibility complex (MHC) results in rejection of the transplanted hepatocytes $(22,23)$. Our results show that hepatocytes can be freely transplanted 
between the R/APfd and the R/APfd-j/j strains and survive more than 180 days in the recipient species. This is further evidence of both major and minor histocompatibility complex identity of the two rat strains.

Mixed lymphocyte reaction. The results of the mixed lymphocyte cultures are given in Table 3. Lymphocyte stimulation was absent in bidirectional MLRs between $\mathrm{R} / \mathrm{APfd}$ and $\mathrm{R} / \mathrm{APfd}-\mathrm{j} / \mathrm{j}$ lymphocytes whereas strong lymphocyte proliferation was observed in MLRs of both R/APfd and R/APfd-j/j lymphocytes with MHCdisparate $\mathrm{BN}\left(\mathrm{RT} 1^{\mathrm{n}}\right)$ lymphocytes.

\section{Biochemical markers}

Comparison of biochemical markers did not reveal any biochemical polymorphism between the R/APfd and R/APfd-j/j strains.

\section{Deficiency of bilirubin UDP-glucuronlytransferase in the $R / A P f d-j / j$ rat}

All R/APfd- $\mathrm{j} / \mathrm{j}$ rats showed the yellowish discoloration of the skin and marked unconjugated hyperbilirubinemia (Table 4 ) that are characteristic for homozygous jaundiced Gunn $(j / j)$ rats. In contrast, bilirubin was barely detectable in the serum of male rats of the control R/APfd strain.

Bilirubin UDP-glucuronyl transferase activity was undetectable in liver homogenate from male and female GUNNXR/Pfd-j/j and $\mathrm{R} / \mathrm{APfd}-\mathrm{j} / \mathrm{j}$ rats. In the normal $\mathrm{R} / \mathrm{APfd}-(+/+)$ controls, the activity for male and female rats was $4.8 \pm 0.1 \mathrm{nmol}$. $(n=4)$ and $6.9 \pm 0.3$ nmol. $(n=4)$, respectively, of bilirubin conjugated per min per $\mathrm{mg}$ of protein. In bile of R/APfd-j/j rats the concentrations of monoconjugates and diconjugates of bilirubin were below the limits of detection of the alkaline methanolysis HPLC method (19) for monoconjugated (10 nM) and diconjugated $(20 \mathrm{nM})$ bilirubin. In rats without defective bilirubin conjugation the biliary concentrations of bilirubin monoester and diester conjugates are of the order of 66 and 150 $\mu \mathrm{M}$, respectively (24).

Table 4. Toral bilirubin concentration ( $\mu M$ ) in the serum of adult rats.

\begin{tabular}{lll} 
& Male rats & Fenall rats \\
\hline R/APfd & $0.4 ; 0.7$ & $0.64 \pm 0.25(n=5)$ \\
R/APfd-j/j & $150 \pm 5(n=4)$ & $192 \pm 27(n=4)$ \\
GUNNXR/Pfd-j/j & $116 \pm 10(n=4)$ & $181 \pm 15(n=4)$ \\
\hline
\end{tabular}

The listed values are means $\pm \mathrm{SD}$. Conjugated bilirubin amounted to $20 \%$ in the serum of the R/APfd rats, whereas none could be detected in the serum of the $\mathrm{R} / \mathrm{APfd}-\mathrm{j} / \mathrm{j}$ rats. Results were obtained by a diazo method except for those for the female R/APfd rats which were obtained by the alkaline methanolysis HPLC procedure. 


\section{Discussion}

The animal model of congenital hyperbilirubinemia, formerly represented by the Gunn rat, has proved its value in various fields of medical research. This model has been of great benefit to the understanding of bilirubin metabolism, enzyme induction and pathophysiology of kernicterus (5). Also, the influence of unconjugated hyperbilirubinemia on fertility $(10)$ and the mechanism of phototherapy of neonatal jaundice $(25,26)$ could be investigated by means of this model. Furthermore, it has been useful in the development of histochemical techniques for specific staining of bilirubin conjugates (27). In recent years, the congenitally jaundiced rat has received increased attention from investigators interested in the treatment of inborn errors of metabolism. Transplantation of hepatic tissue from non-jaundiced rats into the Gunn rat apppeared to lower plasma bilirubin levels $(23,28-31)$. In many studies in which the Gunn rat was used, the reproducibility of results was poor because of the genetic variability of the existing Gunn rat strains and the lack of a nondeficient, but otherwise identical control rat strain. Moreover, transplantation research in the Gunn rat is seriously hampered by the absence of a congenic nondeficient rat strain. Therefore, in the present report on the production and characterization of a new animal model for inherited jaundice caused by defective bilirubin conjugation, demonstration of two features, namely congenicity between the R/APfd-j/j and the $\mathrm{R} / \mathrm{APfd}$ animals and total absence of bilirubin conjugation in the former strain, is of key importance.

Life-long marked hyperbilirubinemia was observed in all $R / A P f d-j / j$ rats, and only unconjugated bilirubin was detectable in serum. In contrast, total bilirubin pigment was lower by a factor of about 200 in the control R/APfd strain and comprised unconjugated as well as conjugated bilirubin. According to Muraca et al. (to be published) the conjugated pigment corresponds to $9 \pm 2 \%$ of total bilirubins. This marked and purely unconjugated hyperbilirubinemia in the R/APfd-j/j strain might have been consistent with defective hepatic uptake as well as with deficient pigment conjugation but ruled out bilirubin overproduction as a sole cause of the hyperbilirubinemia because conjugated bilirubins are detectable, and in fact increased, in the serum of rats loaded with bilirubins (Muraca et al., to be published). Defective bilirubin conjugation in the $R / A P f d-j / j$ rats was demonstrated by two approaches. First, activity of bilirubin UDP-glucuronyl transferase, the enzyme responsible for conjugation, was undetectable in liver homogenate. However, caution is warranted in interpreting the results of this measurement because the enzyme assay has limited sensitivity and the in vitro incubation conditions may not optimally reflect conjugation in the intact animal. Indeed, excretion of considerable amounts of esterified bilirubins in bile has occasionally been observed in patients with Gilbert's syndrome, in whom bilirubin UDP-glucuronyl transferase activity in liver homogenate was undetectable by the standard diazo assay. Therefore a second approach was chosen in which bile of $R / A P f d-j / j$ rats was analyzed for the presence of conjugated bilirubins using a recently developed highly sensitive HPLC method (19). Because 
conjugated bilirubin is excreted rapidly and exclusively via the biliary route, collection and analysis of bile provides an unequivocal approach to assess the conjugation process. Absence of esterified bilirubins in bile unequivocally confirmed that the marked and purely unconjugated hyperbilirubinemia of the R/APfd-j/j rats was caused by complete deficiency of bilirubin UDP-glucuronyl transferase activity.

To produce a congenic strain it is necessary to perform a series of backcrosses with the background strain and to select for conservation of the gene which one wishes to transfer to the background strain. Some genes located in the neighbourhood of the gene to be conserved may be linked to the selected gene and may consequently be replaced with more difficulty by the corresponding genes of the background strain. After 8 backcrosses a congenic strain theoretically already possesses $99.61 \%$ of the genetic material of the background strain, in the case of a recombination frequency of 0.5. Regardless of the actual frequency of recombination, the transferred chromosomal segment which carries the ' $j$ ' gene locus diminishes in length by crossing over at each generation.

As yet, standard surveillance systems for monitoring the genetic integrity of inbred and congenic rat strains are not routinely applied. As a consequence there have been reports in the literature that supposedly 'inbred' rat strains appeared to be noninbred after all, causing serious delay and financial disadvantage for the research programmes involved $(32,33)$. Our results of histocompatibility testing and the absence of biochemical polymorphism are definite proof that the R/APfd and the $\mathrm{R} / \mathrm{APfd}-\mathrm{j} / \mathrm{j}$ strains can safely be considered as congenic after 8 backcross-intercross cycli. At the present moment we have reached the 11th backcross-intercross cycle and will continue this breeding technique to guarantee congenicity further. Recently, Mackenzie and Owens (34) described RHA-j/j and RHA rat strains, claiming that these strains are congenic. However, this claim is not based on adequate genetic monitoring. In addition, their description of the breeding techniques for obtaining these two strains is quite succinct and even suggests that a recombinant inbred strain is involved and not a congenic strain.

In spite of the firm support for congenicity obtained in the present work, small differences in mandible shape were found between the R/APfd and R/APfd-j/j strains (Festing, personal communication, October 1984). Also, the observed difference in growth rate between the two strains seems in contradiction with their congenicity. Both these findings might be ascribed to the toxic effects of unconjugated hyperbilirubinemia.

In conclusion, the enzymatic defect of the GUNNXR/Pfd-j/j rat strain was successfully transferred to the inbred R/APfd rat strain by repeated backcross-intercross mating. This resulted in the new enzyme-deficient $R / A P f d-j / j$ strain. This strain provides a new model of hereditary nonhemolytic unconjugated hyperbilirubinemia, with the congenic R/APfd strain as an ideal control strain without enzyme deficiency. We have already used this new strain in transplantation studies for the treatment of enzyme deficiency disease with good results (35). As is pointed out 
above, the potential of this new inbred strain for various fields of investigation is considerable, especially in the presence of a suitable control strain.

\section{Acknowledgements}

We are grateful to Mr. J. Stessel (Leuven) and Mr. G. Pauwels (Leuven) for breeding and taking care of the animals, to Mrs. I. Daemen (Maastricht), Mr. L. Denolf, Mr. G. Michiels and Mr. R. Michiels (all Leuven) for technical assistance, and to the Belgian Fund for Medical Scientific Research (FGWO) for financial support.

This paper has been published in Laboratory Animals.

Leyten R, Vroemen JPAM, Blanckaert N, Heirwegh KPM.

The congenic normal $R / A P f d$ and jaundiced $R / A P f d-j / j$ rat strains: a new animal model of hereditary nonhemolytic unconjugated hyperbilirubinemia due to defective bilirubin conjugation.

Laboratory Animals 1986; 20: 335.

\section{References}

1. Gunn $\mathrm{CH}$.

Hereditary acholuric jaundice in a new mutant strain of rats.

J Hered 1938; 29: 137.

2. Crigler JF, Najjar VA.

Congenital familial nonhemolytic jaundice with kernicterus.

Pediatrics 1952;10:169.

3. Gunn $\mathrm{CH}$.

Hereditary acholuric jaundice in the rat.

J Can Med Ass 1944; 50: 230.

4. Rabinson R.

Genetics of the Norway rat.

In: International series of monographs in pure and applied biology: Division Zoology. Volume 24 .

Edited by: Kerkut OA. Pergamon Press, Oxford 1965; p. 307.

5. Swarm RL.

Congenital hyperbilirubinaemia in the rat: an animal model for the study of hyperbilirubinaemia. In: Animal models for bionedical research IV. Edited by: Swarm RL. National Acadiemy of Sciences, Washington, D.C. 1971; p. 149.

6. Calabrese EJ.

Hyperbilirubinaemia, Model No. 157.

In: Handbook: Animal models of human disease, vol. 8. Edited by: Jones TC, Hackel DB and Migaki G. Registry of Comparative Pathology, Armed Forces Institute of Pathology, Washington, D.C., 1979.

7. Cornelius $\mathrm{CE}$.

Crigler-Najjar syndrome.

In: Spontaneous animal models of human disease, vol. II. Edited by: Andrews EJ, Ward BC and Altman NH. Academic Press, Londlon $1979 ;$ p. 51.

8. Johnson L, Garcia ML, Figueora $E$, Sarmiento $F$.

Kernicterus in rats lacking glucuronyltransferase. II. Factors which alter bilirubin concentration and frequency of kernicterus.

A.n $\mathbb{W}$ Dis Child 1961; 101: 322 . 
9. Yeary RA.

Embryotoxicity of bilirubin.

Am J Obstet Gyn 1977; 127: 497.

10. Davis RD, Yeary RA.

Impaired fertility in the jaundiced female (Gunn) rat.

Laboratory Animal Science 1979; 29: 739.

11. Altman $\mathrm{PL}_{\mathrm{g}}$ Katz DD.

Inbred and genetically defined strains of laboratory animals, Part 1 , Mouse and rat.

In: Biological handbooks III. Federation of the American Societies for Experimental Biology,

Bethesda, Maryland, US, 1979; p. 237, 258 and 312.

12. Festing MFW.

International index of laboratory animals, 4 th $\mathrm{Ed}$.

Medical Research Council. Laboratory Animal Centre. Carshalton, UK 1980.

13. Festing MFW.

Mutant symbols and designations.

Rat news letter 1982; 9: 3 .

14. Waynforth HB.

Experimental and surgical technique in the rat.

Academic Press, London 1980; p. 187.

15. Vandeputte M, Sobis $\mathrm{H}$, Billiau A, Van de Maele A, Leyten $\mathrm{R}$.

In utero tumor induction by murine sarcona virus (Moloney) in the rat. I. Biological

Characteristics.

Int J Cancer 1973; 11: 536.

16. Seglen PO.

Preparation of isolated rat liver cells.

Methods Cell Biol 1976; 13: 29.

17. Bender $\mathbb{K}$ et al.

Biochemical markers in inbred strains of the rat.

(Rattus norvegicus).

Immunogenetics 1984; 19: 257.

18. Novros JS, Thomas TR, Knoblock EC.

Improved method for accurate quantitation of total and conjugated bilirubin in serum.

Clin Chem 1979; 25: 1891.

19. Muraca M, Blanckaert $\mathrm{N}$.

Liquid-chromatographic assay and identification of mono- and diester conjugates of bilirubin in normal serum.

Clin Chem 1983; 29: 1767.

20. Heirwegh KPM, Van de Vijver M, Fevery J.

Assay and properties of digitonin-activated bilirubin uridine diphosphate glucuronyltransferase from rat liver.

Biochemical Journal 1972; 129: 605.

21. Mito M, Ebata H, Kusano M, Onishi T, Saito T, Sakomoto S.

Morphology and function of isolated hepatocytes transplanted into rat spleen.

Transplantation 1979; 28: 499.

22. Ebata M, Oikawa I, Mito M.

Rejection of allogenic hepatocytes and fetall hepatic tissue transplanted into rat spleen.

Transplantation 1985; 39: 221 .

23. Vroemen JPAM, Blanckaert $N$, Buturman WA, Heirwegh KPM, Kootstra $G$.

Treatment of enzyme deficiency by hepatocyte transplantation in rats.

J Surg Res 1985; 39: 267.

24. Blanckaert $N$.

Analysis of bilirubin and bilirubin mono- and di-conjugates. Determination of their relative amounts in biological samples.

Biochemical Journal 1980; 185: 115.

25. McDonagh AF, Palma LA, Lightner DA.

Blue light and billirubin excretion.

Science 1980; 208: 145. 
26. McDonagh AF, Paima LA, Trull FR, Lightner DA.

Phototherapy for neonatal jaundice. Configurational isomers of bilinubin.

JAm Chem Soc 1982; 104: 6865 .

27. Desmet $W$, Bullens A-M, De Groote J, Heirwegh KPM.

A new diazo reagent for specific staining of conjugated bilirubin in tissue sections.

J Histochem Cytachem 1968; 16: 419.

28. Rugstad HE, Robinson SH, Yannoni C, Tashjian A.H.

Transfer of bilirubin uridine diphosphate-glucuronyl transferase to enzyme-deficient rats.

Science 1970; 170: 553.

29. Mata: AJ, Sutherland DER, Steffes MW et al.

Hepatocellular transplantation for metabolic deficiencies: decrease of plasma bilirubin in Gunn rats.

Science 1976; 192: 892.

30. Groth CG, Arborgh B, Björkén $C$, Sundberg G, Lundgren G.

Correction of thyperbilirubinemia in the glucuronyltransfiferase deficient rat by intraportal hepatocyte transplantation.

Transplant Proc 1977; 9: 313.

31. Sutherland DER, Matas AJ, Steffes MW, Simmons RL, Najarian JS.

Transplantation of liver cells in an animal model of congenital enzyme deficiency disease: the Gunn rat.

Transplant Proc 1977; 9: 317.

32. Scharp DW.

Rejection of islet 'isografts' in a strain of Lewis rats.

Transplantation 1981; 31: 229.

33. Festing MFW.

Genetic contamination of laboratory animal colonies: an increasingly serious problem.

Institute for Laboratory Animal Resources 1982; $25 ; 6$.

34. Mackenzie PI, Owens IS.

Differences in UDP-glucuronyltransferase activities in congenic inbred rats homozygous and heterozygous for the jaundice locus.

Biochemical Pharmacology 1983; 32: 3777.

35. Vroemen JPAM, Buurman WA, Heirwegh KPM, Van der Linden CJ, Kootstra G.

Hepatocyte transplantation for enzyme deficiency disease in congenic rats.

Transplantation 1986; 42: 130. 


\section{Hepatocyte transplantation for enzyme deficiency disease in congenic rats}

\section{Summary}

Long-term effects of hepatocyte transplantation (HTX) in the treatment of enzyme deficiency disease were studied. Congenic enzyme-deficient (R/APfd-j/j) and nonenzyme-deficient ( $R / A P f d)$ rats were used as recipients and donors, respectively. The $R / A P f d-j / j$ rat strain is congenitally deficient of bilirubin uridyldiphosphate (UDP)-glucuronyl transferase.

$\mathrm{R} / \mathrm{APfd}-\mathrm{j} / \mathrm{j}$ rats underwent HTX by intrasplenic injection of $10^{7}$ isolated R/APfd hepatocytes (Group I-A). Another group of R/APfd-j/j rats was treated similarly, but underwent splenectomy after 11 weeks (Group I-B). Controls consisted of $\mathrm{R} /$ APfd $\mathrm{j} / \mathrm{j}$ rats grafted with $10^{7} \mathrm{R} / \mathrm{APfd}-\mathrm{j} / \mathrm{j}$ hepatocytes (Group II), and $\mathrm{R} / \mathrm{APfd}-\mathrm{j} / \mathrm{j}$ rats that underwent a sham operation (Group III).

Total plasma bilirubin (TB) levels were significantly reduced in Groups I-A and I-B during the experiment (both $\mathrm{p}<0.01$ ). In the control groups $\mathrm{TB}$ reduction was not observed. Bile analyses at 30 weeks after HTX showed that in Group I-A $13.7 \pm 2.7 \%$ of total biliary bilirubin was conjugated. In Group I-B a significantly lower fraction was conjugated: $6.6 \pm 1.1 \%(\mathrm{p}<0.05)$. Conjugated bilirubin was not found in bile of Groups 11 and III. Histology showed survival of hepatocytes in all spleens of rats of Groups I-A, I-B and II.

It is concluded that congenic hepatocytes from $\mathrm{R} / \mathrm{APfd}$ donors are not rejected after transplantation into the $R / A P f d-j / j$ rat, and maintain long-term function. Splenectomy does not abolish, but does reduce, the therapeutic effect significantly, indicating that part of the transplanted hepatocytes maintains function in the enzyme-deficient host liver. The congenic $R / A P f d-j / j$ and $R / A P f d$ rat strains represent a new animal model for research in metabolic deficiency disease. 
Experimental hepatocyte transplantation (HTX) is receiving increasing attention as a possible treatment for acute hepatic failure or enzyme deficiency disease. This therapeutic approach is based on the assumption that grafted hepatocytes provide metabolic support for deficient hepatic function(s) (1-8).

Previously we showed that the Gunn rat, a rat strain with unconjugated hyperbilirubinemia due to a hereditary absolute deficiency of bilirubin uridyldiphosphate(UDP)-glucurony』 transferase activity, can be treated by intrasplenic hepatocyte transplantation. However, hepatocytes from nondeficient allogenic donors are rejected by the Gunn rat, which renders the therapeutic effect only transient (9). This rejection of allogenic hepatocytes transplanted into the rat spleen has recently been described in more detail (10). The outcome of previous studies raised the question of whether enzyme deficiency disease could be treated with permanent success if nondeficient donor hepatocytes were not rejected. In the experimetal situation, transplantation of hepatocytes from congenic nondeficient donors would not only give an answer to this question, but would also provide a unique model for the study of DNA-transfer.

In order to obtain a new animal model to study HTX in the treatment of enzyme deficiency without interference of rejection, the recessive "jaundiced" gene of the GUNNXRPfd $/ \mathrm{j} / \mathrm{j}$ rat was transferred to the non-enzyme-deficient R/APfd rat by 8 backcross-intercross cycles. This resulted in the congenic enzyme-deficient $\mathrm{R} / \mathrm{APfd}-\mathrm{j} / \mathrm{j}$ strain ( $\mathrm{j} / \mathrm{j}$ stands for homozygously jaundiced).

In the present work we investigated the long-term results of HTX in the enzymedeficient $\mathrm{R} / \mathrm{APf}-\mathrm{j} / \mathrm{j}$ rat, with special interest in the effects of combined intrasplenic and intraportal HTX.

\section{Materials and methods}

\section{Animals}

Adult R/APfd rats (250-350 g), obtained from the Proefdierencentrum Leuven, University of Leuven, Leuven, Belgium, served as hepatocyte donors. Adult $\mathrm{R} / \mathrm{APfd}-\mathrm{j} / \mathrm{j}$ rats $(300-350 \mathrm{~g})$, from the same supplier, were used as hepatocyte recipients in all experiments. The latter strain was obtained after 8 backcrossintercross cycles of the R/APfd strain with the GUNNXRPfd-j/j strain. As a result, the genetic material of the R/APfd and R/APfd/j/j strains is identical, with the exception of the homozygous "jaundiced" gene in the $R / A P f d-j / j$ rat, and possibly a few genes belonging to the same linkage group 2. The major histocompatibility complex (MHC) determining genes are situated on linkage group 9. Consequently, the R/APfd and R/APfd-j/j strains (hereafter referred to as $R / A$ and $R / A-j / j$ strain, respectively) are congenic and histocompatible. 


\section{Hepatocyfe transplantation}

Isolation of hepatocytes was performed by a modification of the two-step perfusion technique of Seglen (11). After cannulation of the portal vein, the liver was perfused with approximately $250 \mathrm{ml} \mathrm{Ca}{ }^{++}$-free Krebs-Henseleit solution at a flow rate of $20-25 \mathrm{ml} / \mathrm{min}$. The perfusion was gassed with a mixture of $\mathrm{O}_{2}$ and $\mathrm{CO}_{2}(95: 5)$, and kept constantly at $38^{\circ} \mathrm{C}$ and at a pH of 7.4-7.5. During this perfusion the liver was excised without damaging the liver capsule. Subsequently, perfusion was started with a $0.05 \%$ collagenase (type IV, Sigma Chemicals, St. Louis, MO.) and $5 \mathrm{mM} \mathrm{Ca}^{*+}$ ion containing Krebs-Henseleit solution. After $10 \mathrm{~min}$ of collagenase digestion, the swollen liver was disconnected from the perfusion apparatus, and the capsule was removed, thus freeing the isolated liver cells. This initial cell suspension was filtered through a $100-\mu \mathrm{m}$ nylon sieve to eliminate cell clusters and connective tissue debris, and washed three times at $0-4^{\circ} \mathrm{C}$. The cells were then counted and resuspended in Krebs-Henseleit solution containing $10 \mathrm{mg} / \mathrm{ml}$ fatty-acid-free bovine serum albumin (Sigma Chemicals,St.Louis, MO.) to a concentration of $10^{7}$ viable cells per ml. Cell viability was assessed by the trypan blue exclusion test, and ranged from $85 \%$ to $95 \%$. Within $1 \mathrm{hr}$ to $3 \mathrm{hr}$ after hepatocyte isolation, one $\mathrm{ml}$ of the cell suspension (kept on ice) was injected into the spleen of the recipient rat in $30 \mathrm{sec}$. Hillar vessels of the spleen were not clamped. During the intrasplenic injection, suspension was observed flowing off via the splenic veins into the portal vein. In this way, combined intrasplenic and intraportal HTX was performed. Leakage of cell suspension and blood loss was minimized by tamponade of the injection site with a cotton-tip. Ether anesthesia was given to all animals undergoing the operation.

\section{Experimental protocol}

The experimental groups were dividled as follows:

Group I-A: $14 \mathrm{R} / \mathrm{A}-\mathrm{j} / \mathrm{j}$ rats receiving $10^{7} \mathrm{R} / \mathrm{A}$ hepatocytes.

Group 1-B: $8 \mathrm{R} / \mathrm{A}-\mathrm{j} / \mathrm{j}$ rats receiving $10^{7} \mathrm{R} / \mathrm{A}$ hepatocytes, and undergoing splenectomy at 11 weeks post-HTX.

Control groups were as follows:

Group II: $\quad 11 \mathrm{R} / \mathrm{A}-\mathrm{j} / \mathrm{j}$ rats receiving $10^{7} \mathrm{R} / \mathrm{A}-\mathrm{j} / \mathrm{j}$ hepatocytes.

Group III: $\quad 12 \mathrm{R} / \mathrm{A}-\mathrm{j} / \mathrm{j}$ rats undergoing a sham operation (i.e., intrasplenic injection $1 \mathrm{ml}$ Krebs-Henseleit solution).

Blood sampling and measurement of total body weight took place 3 days before HTX, on day 3 post-HTX, weekly till week 4 , and at larger intervals till the end of the experiment at 30 weeks. Total plasma bilirubin (TB) level and hematocrit were determined in each blood sample. Blood samples, obtained at 30 weeks post-HTX, were also examined for the presence of bilirubin glucuronides. During the last weeks of the experiment 24-hr urine collections from the animals were obtained for assay on conjugated bilirubin. At the end of the experiment all animals were provided 
with a bile fistula. In all bile samples concentrations of bilirubin monoglucuronide (BMG), bilirubin diglucuronide (BDG) and total bilirubin, were determined. After bile collection the animals were sacrificed. From each animal tissue specimens were obtained from spleen, liver and right lung for histological examination.

\section{Sampling procedures}

Blood sampling was performed by paraorbital venipuncture under ether anesthesia, and 24 -hr urine samples were obtained by housing the animals in metabolic cages. For bile collection the common bile duct was cannulated with silicone tubing. A bile fistula was created by exteriorizing this silicone cannula at the neck of the animal. This procedure succeeded in 13 (of 14) rats in Group I-A, 6 (of 8) rats in Group I-B, 9 (of 11) in Group 11, and in 11 ( of 12) in Group III. Failures in bile sampling were due to technical problems. Rats were allowed to recover from anesthesia before commencement of bile collection. During this collection ( $3 \mathrm{hr}$ ) rats were able to eat and drink, and to move freely in their cages. Bile was collected in tubes on ice in the semidark.

\section{Analytical methods}

Determination of TB levels was performed by spectrophotometry (American Optical Bilirubinometer, type 10211, Buffalo, NY). Serum, urine, and bile samples were examined for the presence of bilirubin mono- and diglucuronides, using the highly sensitive alkaline methanolysis method combined with high-pressure liquid chromatography, as described by Muraca and Blanckaert (12). This method allows specific detection of small concentrations of bilirubin mono- and diglucuronides in the presence of unconjugated bilirubin (limits of detection 10 and $20 \mathrm{nM}$ respectively).

\section{Histology}

Spleen, liver and lung tissue were fixed in $10 \%$ buffered formaldehyde, and embedded in paraffin. Histological examination of spleen, liver and lung tissue was performed on routine 6- $\mu \mathrm{m}$ tissue sections, stained with hematoxylin and eosin (H\&E) and by the periodic acid Schiff (PAS) procedure.

\section{Statistical analysis}

Comparison of TB levels and total body weight between the experimental groups was performed by repeated measures analysis of variance (two-factor mixed design), completed by post-hoc F-tests (13). For comparison between groups of bile analyses results and liver and spleen weights, the Mann-Whitney U-test was used. Data within the same group were compared by means of the Wilcoxon rank test. 


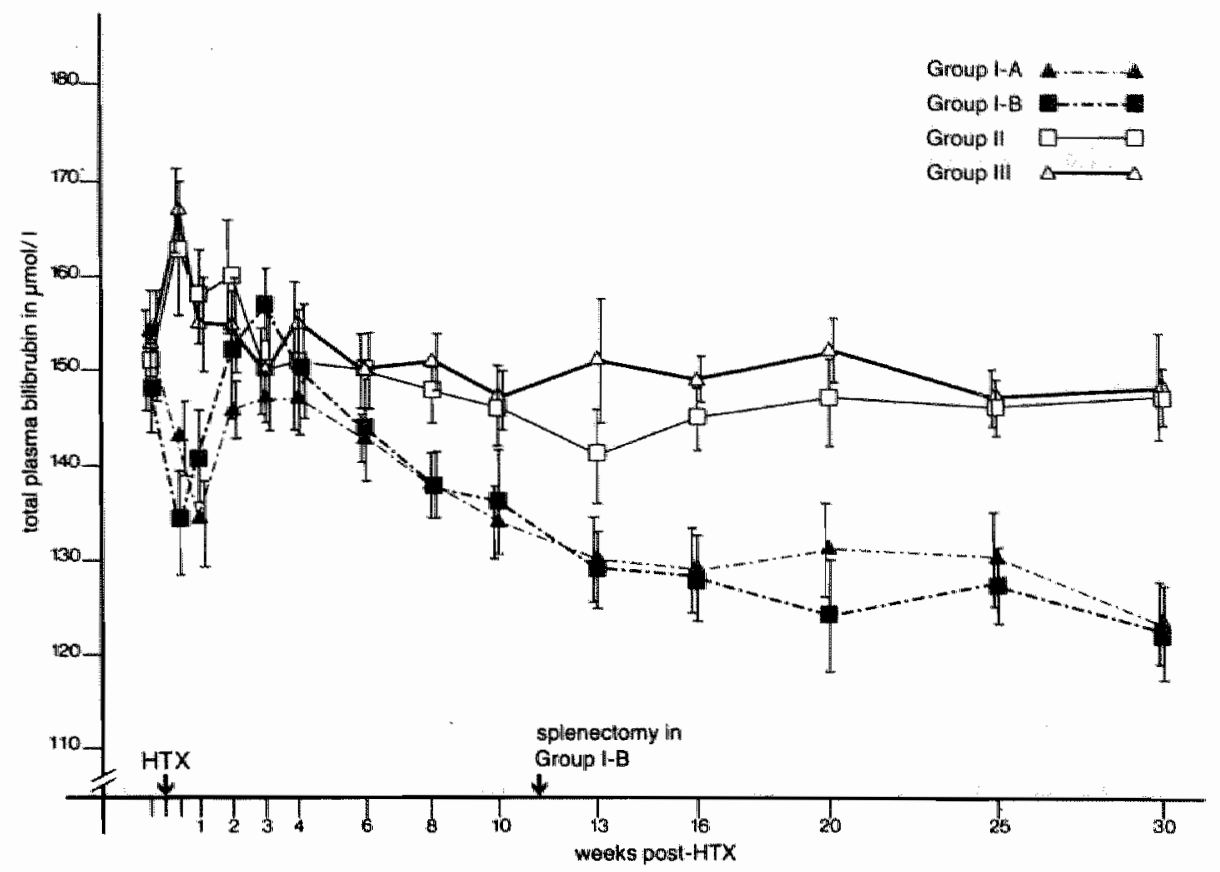

Figure 1. TB levels (mean \pm SEM) during the experiment. Repeated measures analysis of variance demonstrated a significantly different course of $T B$ change between the groups during the experiment $(F(1.6 I)=2.06 ; p<0.005)$. Groups I-A ws. I-B and Groups II ws. III did not differ significantly during the experiment (post-hoc F-test, both $p>0.2$ ). The differences between groups $I-A$ and $I I, I-A$ and $I I I, I-B$ and $I I$, and $I-B$ and III were statistically significant $(p<0.01, p<0.005, p<0.005$ and $p<0.001$, respectively). For discussion see text.

\section{Results}

\section{Assessment of graft function}

TB levels (mean \pm SEM) of the groups are shown in Figure 1. In Groups I-A and I-B, the jaundiced enzyme-deficient rats transplanted with hepatocytes from congenic nondeficient donors, the course of TB levels had a bifasic appearance. During the first week a significant TB decrease was observed in these groups compared with the pre-HTX TB levels $(\mathrm{p}<0.05)$. Thereafter, TB returned to pretransplant levels. From week 6 onwards a gradual decrease in TB was observed in both groups. Splenectomy in the animals of Group I-B at 11 weeks post-HTX did not result in a rise of TB levels. This suggested that donor hepatocytes were functioning not only in the spleen but also in the liver. At the end of the experiment, both Groups $1-\mathrm{A}$ and I-B demonstrated a statistically significant reduction of TB in comparison to pre-HTX values (both $\mathrm{p}<0.01$ ). Mean TB was reduced from $154 \pm 4.5 \mu \mathrm{M}$ to 
$123 \pm 4.3 \mu \mathrm{M}$ in Group I-A, and from $147 \pm 4.1 \mu \mathrm{M}$ to $122 \pm 5.1 \mu \mathrm{M}$ in Group I-B. Jaundiced rats receiving enzyme-deficient hepatocytes or undergoing shamtreatment (Groups II and III, respectively), did not exhibit a decrease in TB levels. Both control groups showed a transient TB increase in the immediate posttransplant period. This phenomenon, observed before in jaundiced rats treated with liver cells from jaundiced donors (9), is most likely caused by increased bilirubin formation and/or additional impairment of bilirubin excretory pathways after surgical trauma or infection.

Anemia due to frequent blood sampling was ruled out as a cause of reduced bilirubin formation and subsequent TB reduction. Hematocrits did not differ significantly between groups during the experiment and remained constant (range 0.45-0.52).

TB reduction caused by the presence of bilirubin UDP-glucuronyl transferase activity after transplantation with hepatocytes from nondeficient donors should imply the formation of bilirubin glucuronides. Biliary excretion of bilirubin glucuronides was demonstrated in all bile specimens from enzyme-deficient rats treated with nondeficient donor hepatocytes (Groups I-A and I-B) at week 30. In Group I-A 13.7 $\pm 2.7 \%$ (mean \pm SEM) of biliary bilirubin was conjugated (range $5.3-42.4 \%$ ), with a mean biliary concentration of bilirubin glucuronides of $1.95 \mu \mathrm{M}$. The mean biliary excretion of conjugated bilirubin in this group amounted to $6.9 \times 10^{-4} \mu \mathrm{mol} / 100 \mathrm{~g}$ b.wt./hr. In Group I-B (treatment identical to that of Group I-A, plus splenectomy at week 11) the mean conjugated fraction of total biliary bilirubin was significantly smaller $(p<0.05): 6.6 \pm 1.1 \%$ (range $4.6-11.8 \%$ ), the mean biliary concentration of bilirubin glucuronides being $1.07 \mu \mathrm{M}$. The mean biliary excretion of conjugated bilirubin was $3.6 \times 10^{-4} \mu \mathrm{mol} / 100 \mathrm{~g}$ b.wt./hr. BMG exceeded BDG excretion in both Groups I-A and I-B (both $\mathrm{p}<0.05$ ). In Group I-A BMG and BDG constituted $8.8 \pm 1.4 \%$ and $4.9 \pm 1.4 \%$, respectively, of total biliary bilirubin. For Group I-B these values were $5.6 \pm 1.0 \%$ and $1.0 \pm 0.1 \%$. In bile from Groups III and III bilirubin glucuronides were not found. These findings provided unequivocal evidence that transplantation of congenic nondeficient hepatocytes results in the persisting activity of the congenitally deficient enzyme in the host. The results of the bile analyses are summarized in Figure 2.

In serum of treated animals, conjugated bilirubin was virtually absent; at 30 weeks, when TB reduction was most significant, traces of conjugated bilirubin were found in only one animal of Group I-A (200 nM BMG and $100 \mathrm{nM} \mathrm{BDG}$ ) and in one animal of Group I-B (400 nM BMG and $540 \mathrm{nM}$ BDG). As expected, conjugated bilirubin was not found in serum samples of Groups II and III. Very low concentrations of unconjugated bilirubin were found in urine of jaundiced rats (range $0-540 \mathrm{nM}$ ). Only in one rat of Group I-A conjugated bilirubin was detected (60 $\mathrm{nM}$ BMG and $30 \mathrm{nM}$ BDG).

\section{Total body weight}

Statistically significant differences among the groups in weight gain were not observed. Considering normal weight gain as an important parameter for the overall state of health in rats, this finding is of importance. 


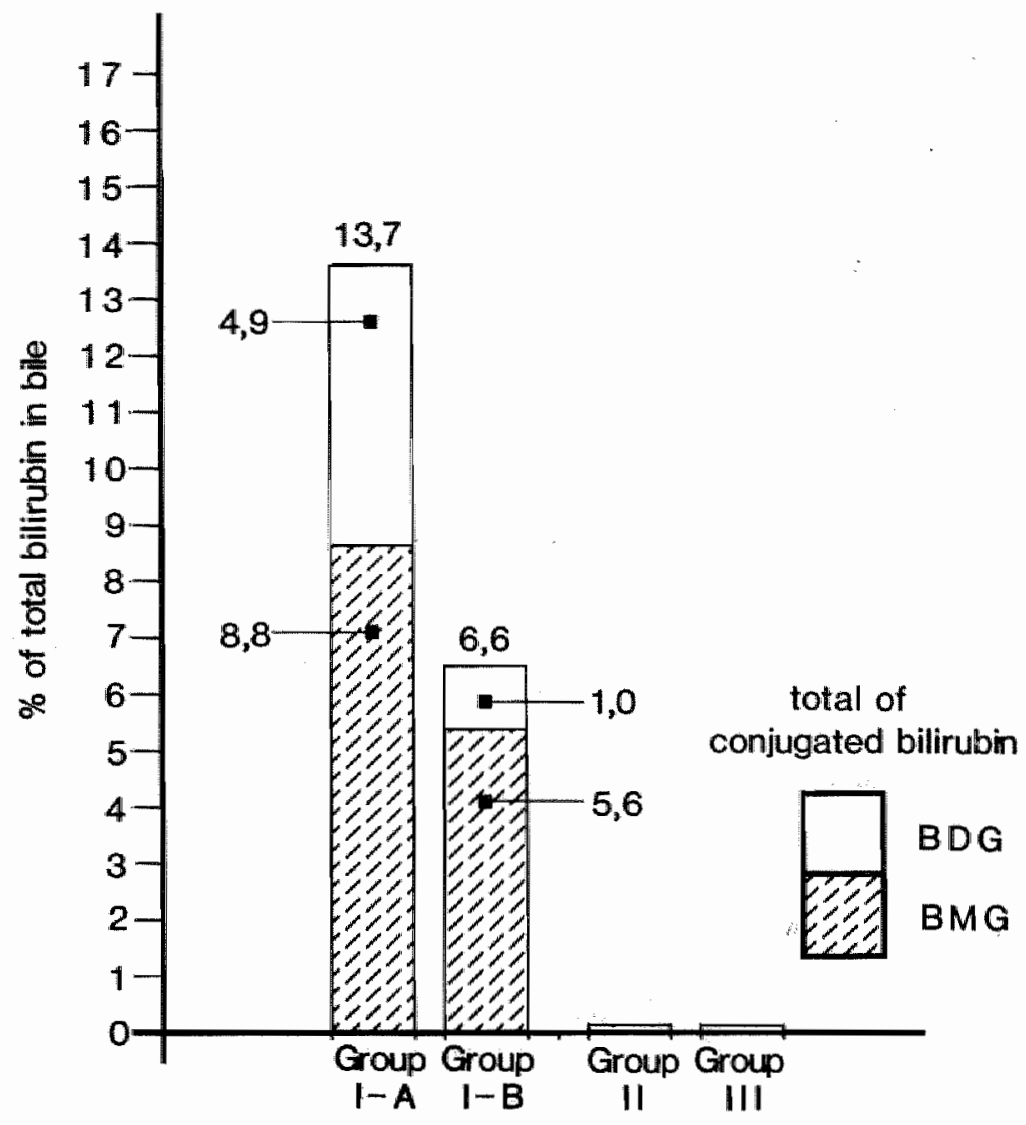

Figure 2. Results of the bile analyses. Conjugated bilirubin was only observed in Group I-A and Group I-B. The quantity of conjugated bilirubin is expressed as the percentage of toral bilirubin in bile. Mean percentages are given. Formation of bilirubin glucuronides was more pronounced in Group I-A than in Group I-B $(p<0.05)$. In both groups the formation of $B M G$ exceeded that of $B D G$ (both $p<0.05$ ).

\section{Observations at autopsy}

At autopsy attention was primarily focused on spleen and liver. Because of the theoretical possibility of migration of donor hepatocytes via the hepatic veins into the pulmonary vascular bed, lung tissue was also histologically studied. In all animals the peritoneal cavity was examined for the presence of ectopic liver tissue, but signs of inoculation of hepatocytes on the peritoneum were not found.

Spleen. The spleens of all rats that received donor hepatocyles (Groups I-A, I-B and II) looked remarkably different from the spleens of the sham-operated animals of Group III, which had a normal macroscopic appearance. Spleens in Groups I-A, and 


\begin{tabular}{lll}
\hline & $\begin{array}{l}\text { liver weight } \\
\text { (in mg) }\end{array}$ & $\begin{array}{l}\text { spleen weight } \\
\text { (in mg) }\end{array}$ \\
\hline Group I-A & $12,117 \pm 667$ & $252 \pm 15$ \\
Group I-B & $12,193 \pm 417$ & $470 \pm 14 \mathrm{c}$ \\
Group II & $13,251 \pm 584$ & $295 \pm 14$ \\
Group III & $12,796 \pm 423$ & $416 \pm 18$ \\
\hline
\end{tabular}

a Liver weights did not differ significantly between groups. Mean values \pm SEM are given.

b Spleen weights in Groups I-A and II were significantly lower than in Groups I-B and III. (all $p<0.01$ )

c Data at splenectomy il weeks post-HTX.

II (having received hepatocytes from normal and deficient donors, respectively) contained white-brown consolidated areas, and were significantly smaller than spleens in Group I-B (data at splenectomy 11 weeks post-HTX) or in Group III (all p<0.01) (Table 1). Group I-B spleens were undergoing changes similar to, though less pronounced than, those in Group I-A spleens, but were not yet reduced in weight, compared with the sham-operated control rats. Histological examination revealed morphologically intact hepatocytes in all spleens of Groups I-A, I-B and II. Most cells retained their typical binuclear appearance (Figure 3). PAS stain demonstrated the presence of glycogen granules in the hepatocyte cytoplasm, indicating the metabolic activity of these cells. Clusters of hepatocytes were dispersed in the red pulp of the spleen. Germinal centers remained intact. Definite histological proof of proliferative activity of transplanted hepatocytes was not observed, although sporadic mitotic figures were found in some animals. In some "hepatized" areas, signs of steatosis were observed (Figure 4). These degenerative changes of the donor hepatocytes occurred in Groups I-A, I-B and II.

Liver. Histological examination of livers did not reveal morphological differences between groups, and showed normal histological features. Grafted hepatocytes could not be distinguished in the recipient livers with the applied histological techniques.

Lung. Hepatocytes were not observed in lung tissue of transplanted rats.

\section{Discussion}

Intraportal infusion of nondeficient isolated hepatocytes has been shown to reduce TB levels in the immunosuppressed Gunn rat $(6,7,8)$. The authors of these previous reports suggested that donor hepatocytes provided bilirubin UDP-glucuronyl transferase activity, resulting in the fall of TB. The excretion of bilirubin conjugates, however, was not demonstrated in these studies. As a consequence, the abovemen- 


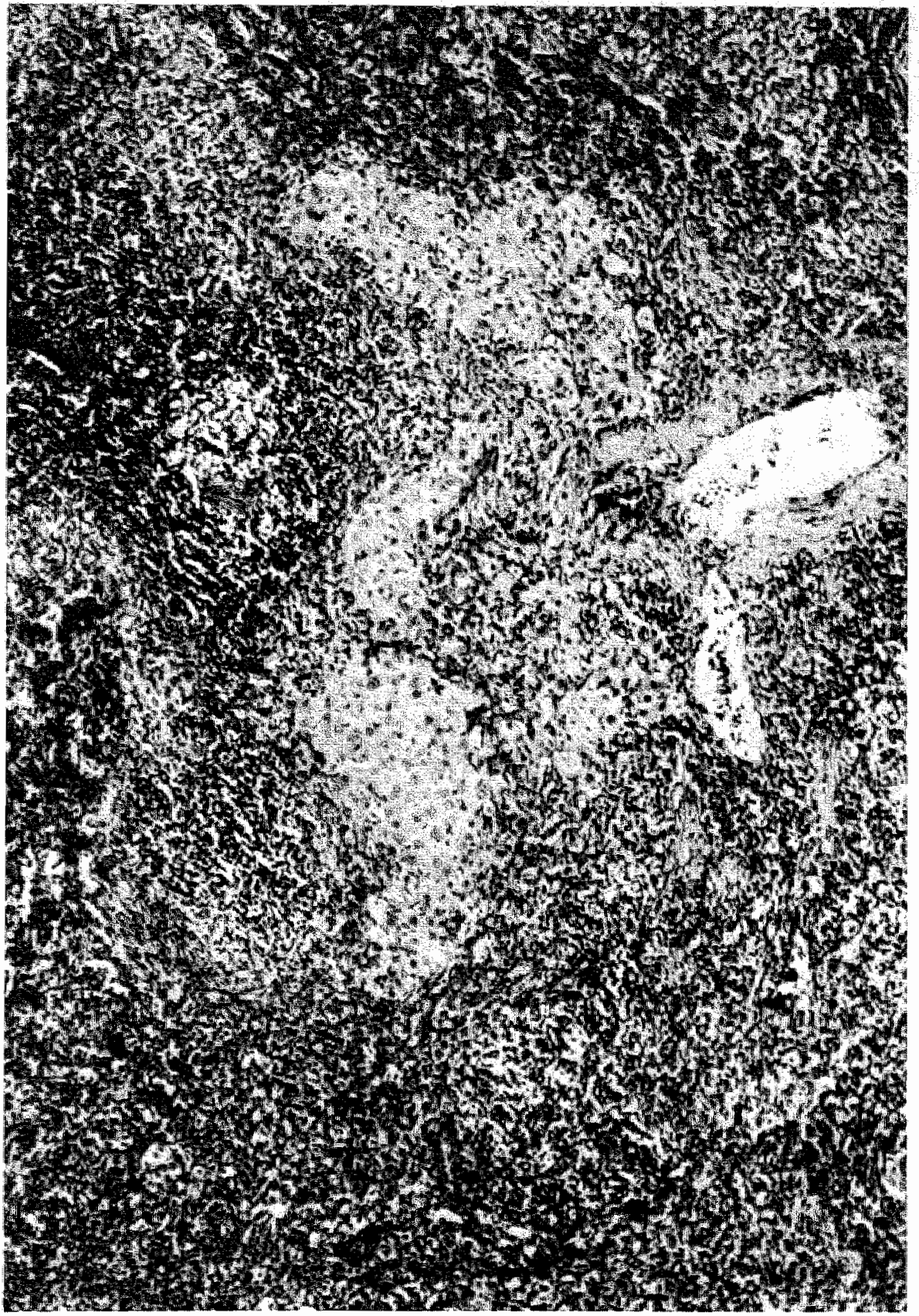

Figure 3. Congenic hepatocytes in the host spleen 30 weeks after transplantation (Group I-A). Morphologically intact hepatocytes are found in the red pulp. Reconfiguration of the typical hepatic structure was not observed. PAS-positive granules into the cytoplasm of the grafted cells indicate intact glycogen metabolism (PAS, $x$ 50). 


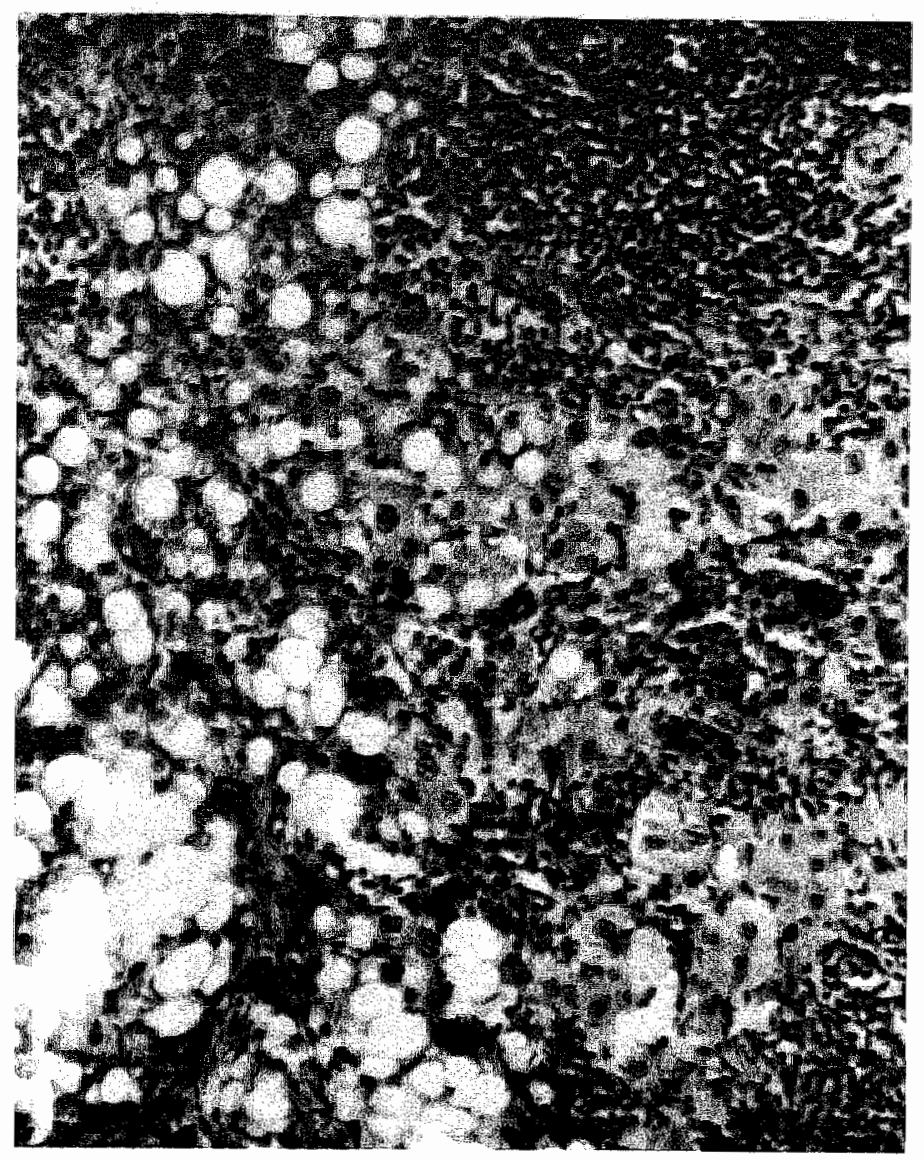

Figure 4. Congenic hepatocytes in the host spleen 30 weeks after transplantation (Group I-A). In some of the areas of the red pulp populated with transplanted hepatocytes, signs of fatty infiltration of the grafted cells occurred. This degenerative change in hepatocytes is also encountered in liver steatosis (H\&E, x 100).

tioned theory was not accepted by others, who believed that functional impairment of the reticuloendothelial system by hepatocyte debris caused prolonged red cell survival, reduced bilirubin formation and a subsequent fall in TB (14). This possibility seems unlikely, since our previous work provided unequivocal evidence for the formation of bilirubin glucuronides by surviving nondeficient donor hepatocytes in the HTX-treated Gunn rat (9). Moreover, here we demonstrate the long-term survival and conjugating capacity of the donor hepatocytes in both spleen and liver of the enzyme-deficient recipient.

From a physiological point of view, intraportal infusion of hepatocyte suspension would seem the most appropriate way of transplantation, offering the isolated hepatocytes the most physiological environment for nidation: i.e., the liver itself. 
However, intraportal HTX confronts the investigator with an important drawback: histological monitoring of graft behaviour is technically difficult, because the donor hepatocytes cannot be easily distinguished from the host liver cells $(15,16)$. In order to avoid this problem in the study of HTX, another suitable recipient organ for isolated hepatocytes was required. It has been documented in syngenic normal rats that the spleen may function as such an organ $(15,16,17)$. After injection into the spleen, hepatocytes inoculate and survive in this organ, and even show proliferative activity. In spite of reports about the necessity of "hepatotrophic factors" from portal blood for unimpaired liver cell function (18), hepatocytes seem to thrive well in the splenic pulp, and remain biochemically active. Intrasplenic HTX allows routine histological monitoring of graft survival, and facilitates anatomical and cytochemical studies of the grafted cells.

These considerations led us to the conclusion that the optimal technique for HTX consisted of a combined intrasplenic and intraportal route of administration of the hepatocyte suspension. This technique was performed by intrasplenic injection of hepatocytes without clamping of the splenic hilar vessels. During infusion one can actually see the suspension flowing through the splenic veins into the portal circulation. Thus, only a part of the hepatocytes remain in the spleen. In this study the long-lasting metabolic activity of intraportally transplanted hepatocytes was assessed by performing a splenectomy in HTX-treated rats several weeks after the transplantation procedure. After splenectomy, bile of transplanted animals of Group I-B still contained bilirubin glucuronides. The quantity of these bilirubin conjugates, however, was only about half of the quantity observed in Group I-B. This provides evidence that a portion of the hepatocytes exerts its function in the spleen, and another part does so in the liver.

The fact that bilirubin glucuronides were not detectable in blood samples of rats of Groups I-A and I-B (with the exception of 2 animals), but were present in bile of all these animals, seems contradictory at first sight. However, it is known that conjugated bilirubin, after intravenous administration to the congenitally jaundiced rat, is rapidly excreted via the biliary route, with a maximal biliary excretion of 5.75 $\mu \mathrm{mol} / 100 \mathrm{~g} \mathrm{~b}$.wt./hr (19). The mean biliary excretions of conjugated bilirubin in Groups I-A and I-B, respectively, were calculated to be 8.300 and 16.500 times less. Therefore, it is conceivable that all conjugated bilirubin, produced by the nondeficient donor hepatocytes, is immediately excreted into bile of the host, and does not reach detectable concentrations in peripheral blood.

The explanation for the bifasic course of bilirubin levels in Groups I-A and I-B is based on the histological data on syngenic HTX of Kusano and Mito $(15,16)$. At the time of transplantation, virtually all hepatocytes are viable with intact metabolic functions. Approximately one week after transplantation many of the donor hepatocytes undergo necrosis, whereas others suffer cellular damage, rendering them temporarily nonfunctional. This sequence of events would explain the initial, but transient TB decrease in our study. After several weeks donor cells would gradually recover, regain their function, and might even start proliferating. This would 
account for the gradual fall in TB observed in this study 6 weeks post-HTX. Our findings at autopsy were largely in accordance with those of other studies, with the exception of steatotic changes in some of the hepatized areas of the spleen after 30 weeks. This observation has not been reported thus far after intrasplenic HTX and is possibly indicative for degenerative changes in the ectopic liver cells, suggesting that the intrasplenic location of donor hepatocytes is not ideal after all. Lung biopsies did not reveal any signs of inoculation of hepatocytes. It is noteworthy in this respect. that a recent report described the successful implantation of isolated rat liver cells in the lung parenchyma by infusing the cell suspension into the jugular vein (20).

In summary, we may conclude from the present data that the R/APfd-j/j and $\mathrm{R} / \mathrm{APfd}$ rat strains provide an excellent model for transplantation research in the treatment of enzyme deficiency disease. Congenic hepatocytes from nondeficient $\mathrm{R} / \mathrm{APfd}$ donors are not rejected after combined intrasplenic and intraportal HTX into the enzyme-deficient R/APfd-j/j rat, and maintain long-term function. This results in the appearance of the missing enzyme activity in the recipient. Splenectomy reduces, but does not abrogate, the therapeutic effect, indicating that part of the transplanted hepatocytes exert their metabolic function in the enzyme-deficient host liver.

In view of these results, HTX from the R/APfd strain to the R/APfd-j/j strain appears to be an excellent model for the study of gene transfer in mammalian cells (21). If gene transfer is performed in isolated R/APfd hepatocytes, and these transfected cells are subsequently transplanted to the $R / A P f d-j / j$ rat, the appearance of bilirubin glucuronides in the transplanted animal will indicate the functional presence of these grafted cells. Determination of the gene product will demonstrate whether or not the gene transfer has been successful. Another approach to the study of gene transfer is the autotransplantation of hepatocytes from the deficient rat, after transfection has provided the correct genetic information for the production of the missing enzyme. In the latter example, HTX with cells from congenic nondeficient donors may serve as a perfect control for the function of the autologous hepatocytes treated by gene transfer.

Sofar, the application of HTX for enzyme deficiency disease has only been studied in the congenitally jaundiced rat. A complete normalization of TB levels has not been obtained by HTX. The required amount of nondeficient hepatocytes is probably too large to ever achieve conjugation of all bilirubin in the recipient. It would, therefore, be interesting to study HTX in a model of enzyme deficiency disease in which only a very small quantity of the missing enzyme activity is required to abolish all manifestations of disease. An example of such disease in the human is phenylketonuria. The presence of $5 \%$ of the normally available quantity of phenylalanine hydroxylase (a liver enzyme) would be sufficient for total cure in the phenylketonuric child (22). Unfortunately, an animal model of such disease is not available.

We expect that HTX, possibly in combination with gene transfer, will offer perspectives for future clinical use in - as yet - uncurable enzyme deficiency diseases. 


\section{Acknowledgements}

The authors wish to thank Mr. G. Michiels (Leuven) and Mr. P. Willems (Maastricht) for technical assistance, Mr. C. Voskamp (Maastricht) for preparing the figures, and Mrs. K. Spronck (Maastricht) for typing the manuscript. Ir. R. Leyten (Leuven) kindly provided the laboratory animals.

This paper has been published in Transplantation.

Vroemen JPAM, Buurman WA, Heirwegh KPM, Van der Linden CJ, Kootstra G. Hepatocyte transplantation for enzyme deficiency in congenic rats.

Transplantation 1986; 42: 130 .

\section{References}

1. Sutherland DER, Numata M, Matas AJ, Simmons RL, Majarian JS.

Hepatocellular transplantation in acute liver failure.

Surgery 1977; 82: 124.

2. Makowka L, Rotstein LE, Falk RE, et al.

Allogeneic and xenogeneic hepatocyte transplantation in experimental hepatic failure.

Transplantation 1980; 30: 429 .

3. Makowka $L$, Rotstein $L E_{\text {, }}$ Falk RE, et al.

Studies into the mechanism of reversal of experimental acute hepatic failure by hepatocyte transplantation.

Can J Surg 1981; 24: 39.

4. Minato M, Houssin D, Demma $I_{\text {, et al. }}$

Transplantation of hepatocytes for treatment of surgically induced hepatic failure in the rat.

Eur Surg Res 1984; 16: 162.

5. Cuervas-Mons V, Cienfuegos JA, Maganto P, et al.

Time-rellated efficacy of liver cell isografts in fulminant hepatic failure.

Transplantation 1984; $38: 23$.

6. Matas AJ, Sutherland DER, Steffes MW et al.

Hepatocellular transplantation for metabolic deficiencies: decrease of plasma bilirubin in Gumn rats.

Science 1976; 192; 892 .

7. Sutherland DER, Matas AJ, Steffes MW, Simmons RL, Najarian JS.

Transplantation of liwer cells in an animal model of congenital enzyme deficiency disease: the Gunn rat.

Transplant Proc 1977; 9: 317 .

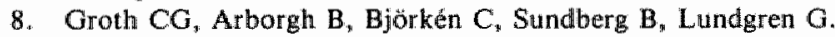

Correction of hyperbilirubinaemia in the glucuronyltransferase-deficient rat by intraportal hepatocyte transplantation.

Transplant Proc 1977; 9: 313.

9. Vroemen JPAM, Blanckaert N, Buurman WA, Heirwegh KPM, Kootstra G.

Treatment of enzyme deficiency by hepatocyte transplantation in rats.

J Surg Res: 1985; 39: 267 .

10. Ebata H, Iwao O, Mito M.

Rejection of allogeneic hepatocytes and fetal hepatic tissue transplanted into the rat splleen.

Transplantation 1985; 39: 221 .

11. Seglen PO.

Preparation of isolated rat liver cells.

Methods Cell Biol 1976; 13: 29. 
12. Muraca M, Blanckaert $N$.

Liquid-chromatographic assay and identification of mono- and diester conjugates of bilirubin in normal serum.

Clin Chem 1983; 29: 1767.

13. Bruning $\mathrm{JL}$, Kintz $\mathrm{BL}$.

Computational Handbook of Statistics. 2nd ed. Glenview, Llinois: Scott, Foresman and Company, 1977.

14. Woods R.J, Parbhoo SP.

An explanation for the reduction in bilirubin leveis in congenitally jaundiced Gunn rats after transplantation of isolated hepatocytes.

Eur Surg Res 1981; 13:278.

15. Kusano $\mathrm{M}$, Mito $\mathrm{M}$.

Observations on the fine structure of long-survived isolated hepatocytes inoculated into rat spleen. Gastroenterology 1982;82:616.

16. Mito M, Ebata H, Kusano M, Onishi T, Saito T, Sakamoto S.

Morphology and function of isolated hepatocytes transplanted into rat spleen.

Transplantation 1979; $28: 499$.

17. Woods RJ, Fuller BJ, Attenburrow VD, Nutt LH, Hobbs KEF.

Functional assessment of hepatocytes after transplantation into rat spleen.

Transplantation 1982; 33: 123 .

18. Starzl TE, Francavilla A Halgrimson CG et al.

The origin, hormonal structure and action of hepatotrophic substances in portal venous blood. Surg Gynecoll Obstet 1973; 137: 179 .

19. Arias LM, Johnson L, Wolfson S.

Biliary excretion of injected conjugated and unconjugated bilirubin by normal and Gunn rats.

Am J Physiol 1961; 200: 1091.

20. Selden $C$, Gupta $S$, Johnstone $R$, Hodgson HJF.

The pulmonary vascular bed as a site for implantation of isolated liver celis in inbred rats.

Transplantation $1984 ; 38: 81$.

21. Kucherlapati R, Skoultchi AI.

Introduction of purified genes into mammalian cells.

CRC Crit Rev Biochem 1984; 16: 349.

22. Bartholomé $\mathbb{K}$.

Genetics and biochemistry of the phenylketonuria-present state.

Hum Genet 1979; 51: 241. 


\section{CHAPTER 5}

\section{Transplantation of isolated hepatocytes into the pancreas}

\section{Summary}

In previous research into HTX the spleen was the preferred acceptor organ for isolated donor hepatocytes. In this study the pancreas was tested as an acceptor organ for hepatocyte transplantation (HTX). HTX into the pancreas or spleen was performed by injection of $10^{7}$ isolated hepatocytes into the parenchyma of these organs.

Intrapancreatic hepatocytes showed good viability three months after syngenic HTX as assessed by histological and immunocytochemical parameters. Definite proof of sustained metabolic activity of normal hepatocytes, 3 months after transplantation into the pancreas of congenitally jaundiced rats, was obtained by demonstration of bilirubin conjugates in bile of the recipients: $4.0 \%$ of total biliary bilirubin was conjugated. Intrasplenic HTX, however, was more effective and resulted in a conjugated fraction of $17.7 \%$ of total biliary bilirubin $(p<0.001)$. Reduction of total plasma bilirubin was significant in both methods, but more pronounced in intrasplenic HTX. Bile drainage from the hepatocellular transplant via the pancreatic excretory system into the gut was not observed: conjugated bilirubins were not found in pancreatic juice of HTX-treated jaundiced rats. Intrapancreatic HTX did not adversely affect the host rat; evidence of pancreatitis or diabetes was not found.

It is concluded that the pancreas is a suitable acceptor organ for HTX. However, intrapancreatic HTX appears to be less effective than intrasplenic HTX in the treatment of enzyme deficiency disease. 


\section{Introduction}

The results of hepatocyte transplantation (HTX) in animal models of inherited metabolic disease and severe liver failure have offered perspectives for the future clinical use of this new transplantation technique (1-11). Functional survival of ectopically transplanted isolated hepatocytes has been described in spleen $(5,12-15)$, lung (16) and peritoneum $(17,18)$. It was shown that liver cell rearrangement after HTX resulted in partial reconfiguration of the hepatic microstructure, with formation of intercellular bile canaliculi and sinusoids $(13,14)$. Ample proof was provided that transplanted hepatocytes retain many, and possibly all, of their metabolic functions $(4,5,12-15,17,18)$. In previous work it was shown that in the absence of an excretory route, bile products from transplanted isolated hepatocytes were secreted into the portal circulation, and were eventually excreted by the host liver into the biliary system $(4,5,15,17,18)$.

In theory, the pancreas would be an appropriate acceptor organ for the transplantation of isolated hepatocytes. This organ, of the same embryologic origin as the liver, is well vascularized and contains the hepatotrophic factors necessary for sustained viability of the transplanted hepatocytes $(19,20)$. HTX into the pancreas might even develop into a useful remedy for end stage cirrhosis, if neoformation of bile ductules by hepatocytes transplanted in the pancreas would result in bile drainage into the excretory system of this organ.

In this study we tested the pancreas as an acceptor organ for HTX. Survival and metabolic activity of the transplanted hepatocytes were investigated. Intrapancreatic and intrasplenic HTX were compared in regard to metabolic activity of the transplanted hepatocytes. This was done in a rat model of enzyme deficiency that was recently described (21). In addition, we investigated whether bile from the hepatocellular transplant was excreted into the gut via the excretory ducts of the pancreas.

Materials and methods

\section{Animals}

Adult rats of the congenic strains $\mathrm{R} / \mathrm{APfd}$ and $\mathrm{R} / \mathrm{APfd}-\mathrm{j} / \mathrm{j}$ were used as hepatocyte donors and recipients. The inbred $R / A P f d$ rat strain is of Wistar origin with normal liver metabolism. The congenic $R / A P f d-j / j$ rat is histocompatible with the firstmentioned strain, but suffers from a congenital enzyme deficiency of bilirubin uridyldiphosphate glucuronyl transferase (BGT) (21). It does not concern a malfunctioning enzyme, but the total absence of the BGT enzyme protein (22). This rat strain consequently exhibits an unconjugated hyperbilirubinemia, due to the absolute inability to conjugate bilirubin with glucuronic acid $(j / j$ stands for homozygously jaundiced). 
All operative and sampling procedures were performed under ether anesthesia.

\section{Hepatocyte transplantation}

Isolation of donor hepatocytes was performed according to the two-step collagenase digestive method of Seglen (23). The initial cell suspension was filtered through $100-\mu \mathrm{m}$ nylon mesh, washed three times in Krebs-Henseleit solution and suspended to a concentration of $10^{7}$ viable hepatocytes per ml. Viability of the resulting cell suspension ranged from $85-95 \%$, as assessed by the trypan blue exclusion test.

Transplantation of isolated hepatocytes was performed using a left subcostal incision, through which spleen and pancreas were exteriorized. HTX into the pancreas was achieved by subcapsular injection of $10^{7}$ viable hepatocytes into the pancreatic parenchyma through a 25 -gauge needle, using 7-10 injection sites randomly distributed over the pancreas. Leakage of hepatocyte suspension and hemorrhage were negligible. Manipulation of the pancreas was limited as much as possible. HTX into the spleen consisted of a single injection of one $\mathrm{ml}$ of hepatocyte suspension into the splenic parenchyma. Tamponade with a cotton-tip provided adequate hemostasis.

\section{Experimental protocol}

In order to study the survival of transplanted isolated hepatocytes into the pancreas $6 \mathrm{R} / \mathrm{APfd}$ rats received a hepatocellular transplant, and were sacrificed at twelve weeks post-transplantation. Postmortem investigations consisted of macroscopic examination of the intra-abdominal organs and histological examination of pancreas and liver. Pancreas and liver weights were compared with those of untreated control rats.

The metabolic activity of transplanted isolated hepatocytes into the pancreas was tested in the animal model described above, which we have also used in earlier studies of HTX. The long-term metabolic function of nondeficient R/APfd hepatocytes transplanted into the BGT-deficient $R / A P f d-j / j$ rat could be assessed by demonstrating the appearance of bilirubin mono- and diglucuronides (BMG and BDG, respectively) in bile of the recipient (5). This animal model also offered the possibility to investigate whether or not functional neoformation of bile ductules occurred with drainage into the excretory apparatus of the pancreas.

In order to demonstrate metabolic activity of the isolated hepatocytes transplanted into the pancreas, $36 \mathrm{R} / \mathrm{APfd}-\mathrm{j} / \mathrm{j}$ rats were treated as follows:

Group I: $\quad \mathrm{R} / \mathrm{APfd}-\mathrm{j} / \mathrm{j}$ rats received a hepatocellular transplant of $10^{7} \mathrm{R} / \mathrm{APfd}$ hepatocytes into the pancreas $(n=12)$.

Group II: R/APfd- $\mathrm{j} / \mathrm{j}$ rats were treated by HTX into the spleen, receiving $10^{7}$ R/APfd hepatocytes $(n=8)$. 
Group III: $\mathrm{R} / \mathrm{APfd}-\mathrm{j} / \mathrm{j}$ rats received a hepatocellular transplant into the pancreas, with donor hepatocytes originating from $R / A$ Pfd- $j / j$ rats $(n=8$, negative control group).

Group IV: $R / A P f d-j / j$ rats that underwent a sham-operation, i.e. the injection of $1 \mathrm{ml}$ of Krebs-Henseleit solution into the pancreas $(\mathrm{n}=8$, second negative control group).

Before HTX, total body weight, serum amylase (SA) and total plasma bilirubin (TB) were measured. At 12 weeks post-HTX these parameters were recorded again. Urine specimens were screened for the presence of glucose. In addition, bile and pancreatic juice of each animal were sampled separately to be analyzed on the presence of the bilirubin conjugates BMG and BDG. After these sampling procedures the animals were sacrificed. Pancreas, spleen and liver were weighed and fixed for histological examination.

\section{Sampling procedure}

Blood samples were taken by paraorbital venipuncture. For the simultaneous collection of bile and pancreatic juice, a midline abdominal incision was used. Bile was collected by cannulation of the proximal common bile duct with silicone tubing. For the collection of pancreatic juice a duodenal segment of $3-4 \mathrm{~cm}$ was isolated by tying a ligature around the pyloric region and the distal duodenum. This isolated duodenal segment was thoroughly rinsed with saline solution in order to remove all nutritional debris and remainder of bile. Hereafter, silicone tubing provided with multiple holes at its tip was inserted into this isolated gut segment in order to collect pancreatic juice. The bile and pancreatic juice draining silicone cannulae were tunneled subcutaneously, and exteriorized at the neck of the animal. Before commencement of the sampling procedure, animals were allowed to recover from anesthesia and were permitted to move freely in their cages. During a period of approximately 3 hours bile $(3-4 \mathrm{ml})$ and pancreatic secretions $(1.5-2.5 \mathrm{ml})$ of each animal were collected on ice in the semidark. Technical problems in bile sampling were not encountered. Collection of pancreatic juice failed in 3 instances in Group I, 2 in Group II, 2 in Group III and 3 in Group IV.

\section{Analytical methods}

Assays of bile and pancreatic excretions on the presence of conjugated bilirubins were performed by the alkaline methanolysis method in combination with high-pressure liquid chromatography, as described by Muraca and Blanckaert (24). This method is extremely sensitive, with limits of detection of $10 \mathrm{nM}$ BMG and 20 nM BDG in the presence of unconjugated bilirubin. Total plasma bilirubin (TB) levels were measured by spectrophotometry (American Opticals Bilirubinometer, type 10211, Buffalo, NY). Serum amylase (SA) levels were determined by spectrophoto- 
metry, using 2-chloro-4-nitrophenyl- $\beta$-D-maltoheptaoside as substrate (25). Screening for abnormal urinary loss of glucose was performed with the Gluketurtest (Boehringer, Mannheim, FRG). The level of glucose detection of this test is less than $2 \mathrm{mM}$.

\section{Histology}

Pancreas, spleen and liver of all animals were fixed in 10\% buffered formaldehyde and embedded in paraffin. Histological examination was performed on $4-\mu \mathrm{m}$ sections, stained with hematoxylin and eosin (H\&E) and according to the periodlic acid Schiff (PAS) method. Selected slides were stained for reticulin. Immunostaining of basement membranes was performed on rehydrated paraffin sections using a specific anti-type IV collagen antibody and a peroxidase-antiperoxidase method (26).

\section{Statistical analysis}

For comparison of data between groups the Mann-Whitney U-test was used. Data within the same group were compared by means of the Wilcoxon signed-ranks test (27).

Results

\section{Histological observations}

On macroscopic inspection at 12 weeks post-HTX the pancreas of rats that underwent HTX into this organ was of normal size, but possessed a little more solid appearance, instead of the typical loose organ structure. Another, but only minor irregularity was the formation of local fibrous adhesions as a result of the operative procedure itself, as such also present in sham-operated rats. On gross examination there were neither signs of ectopic liver tissue in the pancreas, nor signs of pancreatic disease, such as inflammatory edema. Liver and pancreas weights did not differ from those of untreated control rats.

Histological examination of the pancreas of normal Wistar (R/APfd) rats that received syngenic hepatocytes into the pancreas showed survival of the donor liver cells in all animals. The surviving hepatocytes showed good viability and were morphologically identical to normal hepatocytes. Cell size and structure on light microscopic examination were shown to be normal. Transplanted hepatocytes often showed a binuclear appearance (Figure 1). Signs of mitotic activity were not observed. Histochemical PAS staining of polysacharides revealed the presence of the bright purple staining glycogen granules in the cytoplasm of surviving donor hepatocytes, indicating intact carbohydrate metabolism 12 weeks after transplantation. Surviving hepatocytes were arranged in clusters, ranging in number (on section) from a dozen to a hundred cells. Sometimes these clusters took the shape of hepatic 


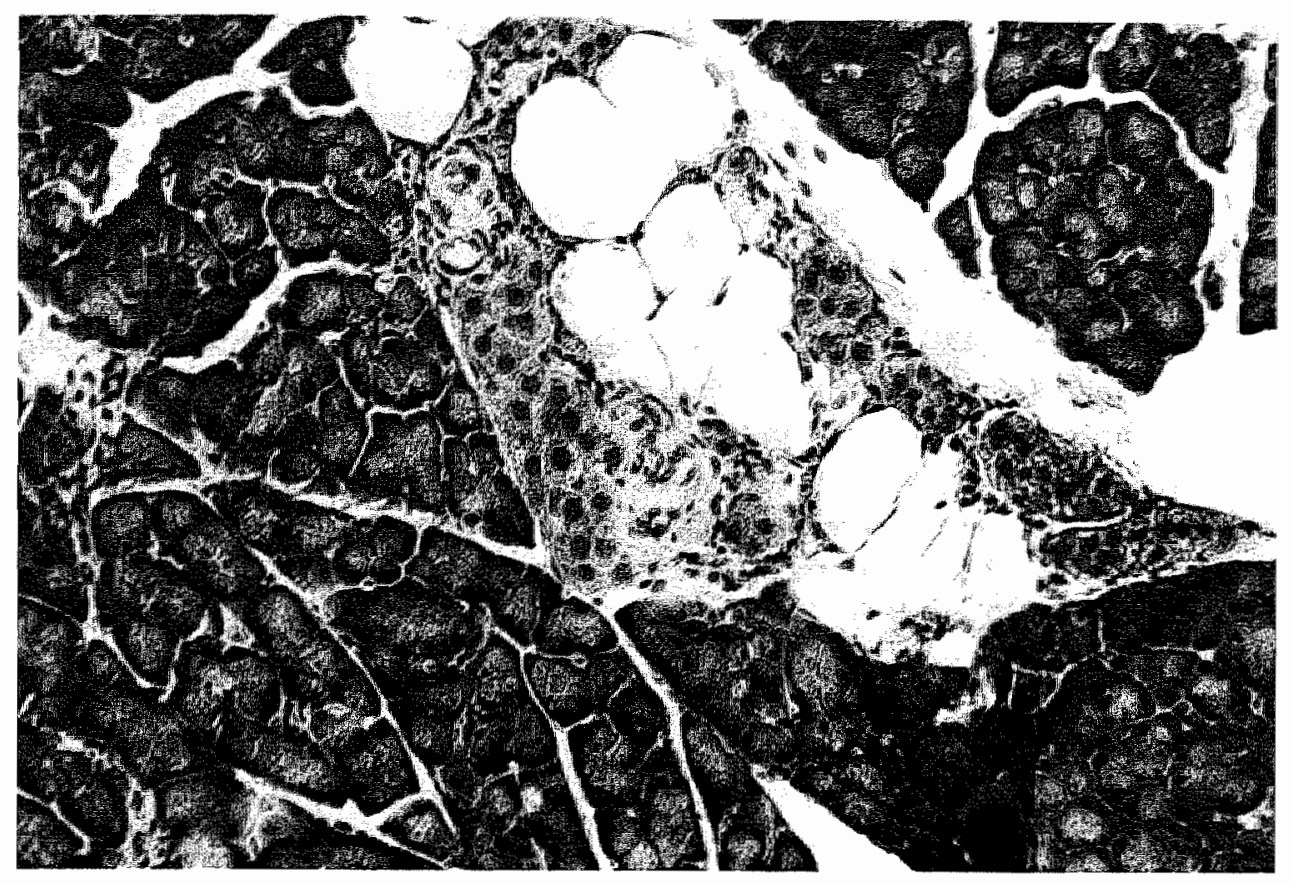

Figure 1. Survival of syngenic donor hepatocytes in the pancreatic parenchyma 3 months after HTX into the pancreas of normal rats. The hepatocytes are morphologically idertical to normal hepatocytes, unaltered in size and often with a binuclear appearance. Signs of proliferative activity are not observed. Degenerative changes (loss of cell mass, densification of cytoplasm and pycnotic nuclet) are visible in liver cells at the periphery of the hepatocellular aggregate (H\&E, $x$ 75).

cell cords, but a real reconfiguration of the lobular hepatic structure was never seen. Most often, the hepatocellular aggregates were found in the periphery of the pancreatic lobules, lying in or along the interlobular septa of connective tissue in the vicinity of blood vessels and adipose cells (Figure 2). Only occasionally, aggregates were found in the middle of the lobules amongst pancreatic acini. There was a definite reticulin pattern at the interface between the periphery of the clusters and the stroma, as well as around many hepatocytes (Figure 3). Small vascular structures were visible in the hepatocyte aggregates, the basement membranes of which exhibited immunoreactivity for type IV collagen. This was an indication that neovascularization of the hepatocellular graft occurred. As could be expected, basement membranes were absent around the transplanted hepatocytes (Figure 4). Degenerative changes of the transplanted hepatocytes, e.g. cell shrinkage with densification of cytoplasm or pycnosis, were observed sometimes at the periphery of the hepatocellular aggregates. The recipient pancreas showed an undisturbed tubulo-acinar histological pattern, with normal islets of Langerhans. Histological signs of pancreatitis were absent. Leucocyte infiltration, diffuse or restricted to the direct environment of the donor cell aggregates was not observed. 


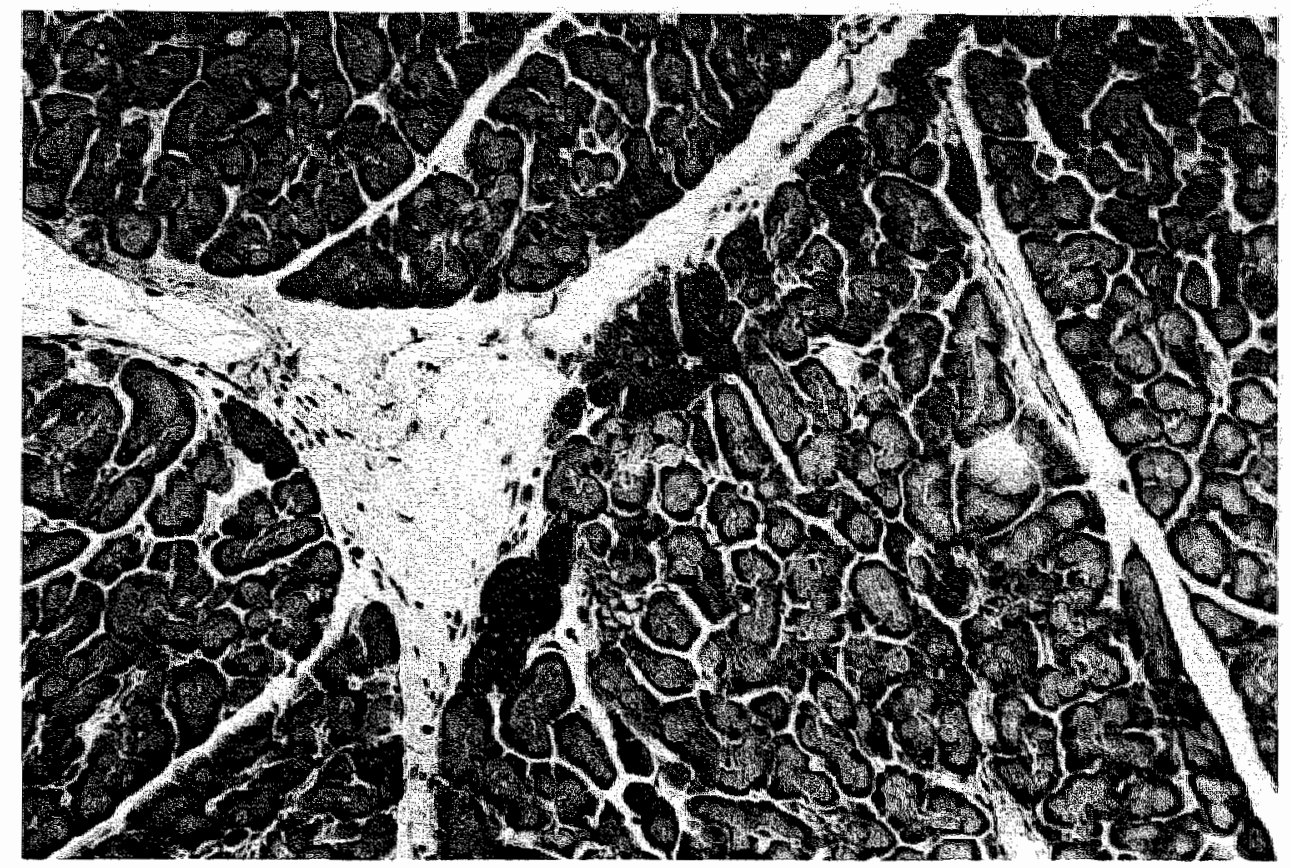

Figure 2. Hepatocytes from normal donor rats transplanted into the pancreas of congenic hyperbilirubinemic BGT-deficient rats show intact morphological features and good viability. The cytoplasma of these donor hepatocytes contains PAS-positive staining granules as evidence of intact glycogen metabolism (PAS, $x$ 50).

Histology of the pancreas of jaundiced BGT-deficient R/APfd $/ \mathrm{j} / \mathrm{j}$ rats that received an injection of hepatocyte suspension from normal congenic $R / A P f d$ donor rats (Group I) or from enzyme-deficient $R / A P f d-j / j$ rats (Group III) into the pancreas showed survival of the donor liver cells. Morphological features of these cells and their arrangement and distribution over the pancreas were as outlined above. The incidence of degenerative changes in transplanted hepatocytes seemed to be slightly increased in the hyperbilirubinemic recipient rat.

Survival of normal (R/APfd) hepatocytes, transplanted into the spleen of enzymedeficient $R / A P f d-j / j$ rats (Group II) was observed in all recipients. The histological appearance of intrasplenic donor hepatocytes has been described in recent work (5) (see Chapter 4).

The pancreas and spleen of sham-treated R/APfd-j/j rats (Group IV) showed normal histological features.

Metabolic activity of isolated hepatocytes transplanted into the pancreas

The long-term metabolic function of normal hepatocytes transplanted in the BGTdeficient rat can be assessed accurately by determination of the appearance of the 


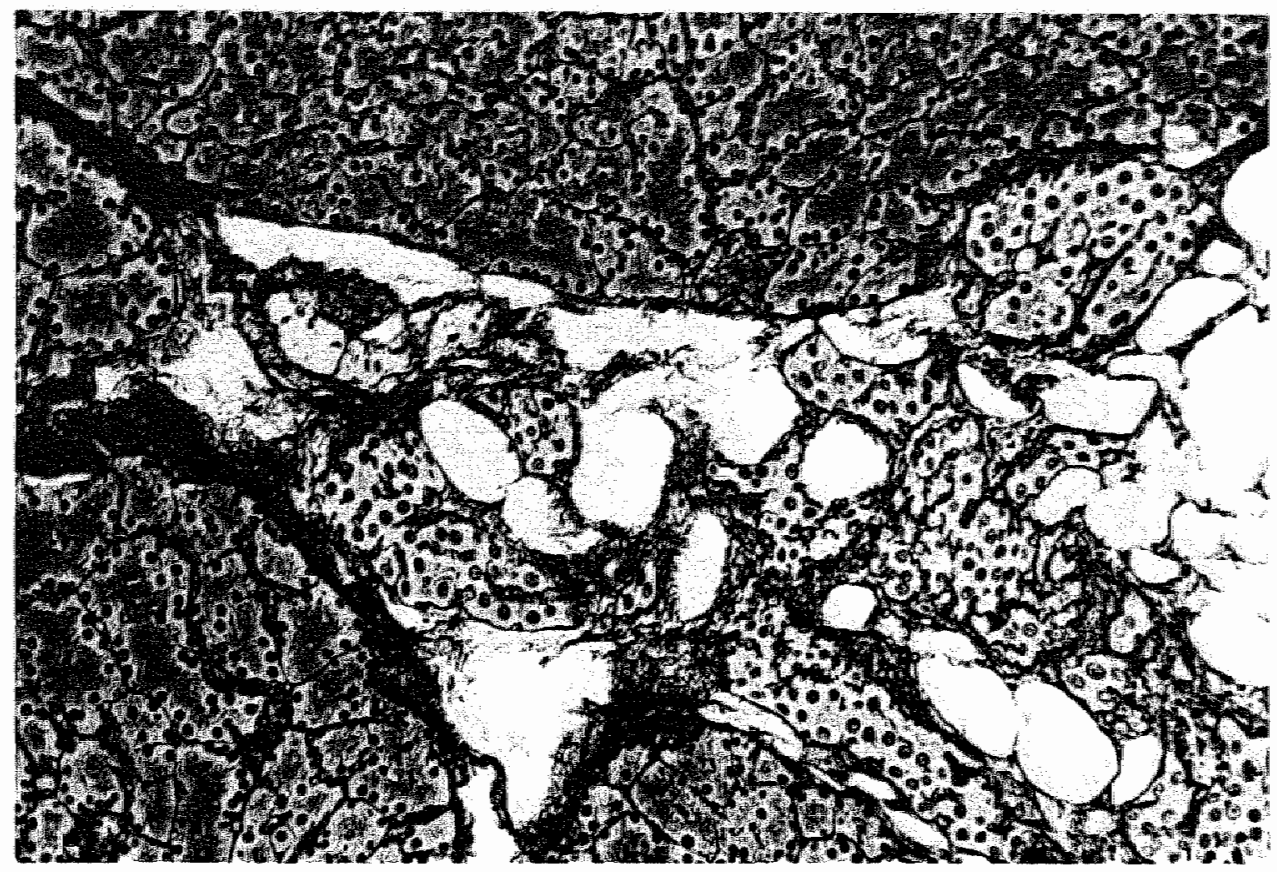

Figure 3. Hepatocyte clusters embedded in interlobular fat tissue. The clusters and many individual hepatocytes are surrounded by reticulin fibers (reticulin siain, $x 50$ ).

products of BGT, namely $\mathrm{BMG}$ and $\mathrm{BDG}$, in the recipient animal $(4,5,17,18)$. The quantity of billirubin glucuronides measured in bile of the experimental groups are shown in. Figure 5.

In Group I, it was evident that the surviving normal hepatocytes transplanted into the pancreas of BGT-deficient rats were metabolically active. BMG and BDG were both excreted in bile of these rats. In this group $4.0 \pm 0.7 \%$ (mean \pm SEM) of all biliary excreted bilirubin was glucuronized (range 1.9\% $-7.5 \%$ ). The relative quantities of $\mathrm{BMG}$ and $\mathrm{BDG}$ constituted $2.3 \pm 0.3 \%$ and $1.7 \pm 0.4 \%$, respectively. The mean biliary concentration of glucuronized bilirubin (i.e. BMG plus BDG) amounted to $1.62 \mu \mathrm{M}$, with a mean excretion rate of conjugated bilirubin of $5.2 \times 10^{-4}$ $\mu \mathrm{mol} / 100 \mathrm{~g} \mathrm{~b}$.wt/hr. In Group II, BGT-deficient rats that received normal hepatocytes in the spleen, $17.7 \pm 1.3 \%$ of total biliary bilirubin appeared to be glucuronized (range 14.7\% - 20.5\%), the BMG fraction being $10.2 \pm 0.3 \%$, versus a BDG fraction of $7.5 \pm 0.9 \%$. Mean biliary concentration of conjugated bilirubin was $6.56 \mu \mathrm{M}$, mean excretion rate of conjugated bilirubin $19.6 \times 10^{-4} \mu \mathrm{mol} / 100 \mathrm{~g} \mathrm{~b} . \mathrm{wt} / \mathrm{hr}$. The difference in biliary excretion of bilirubin glucuronides between Groups I and II was statistically significant $(\mathrm{p}<0.002)$. In both groups the formation of BMG significantly exceeded that of BDG (both $\mathrm{p}<0.01$ ). In control Groups III (transplantation of BGT-deficient hepatocytes into the pancreas of BGT-deficient rats) and IV (sham-treated BGT-deficient rats) bilirubin glucuronides were not found in bile. 


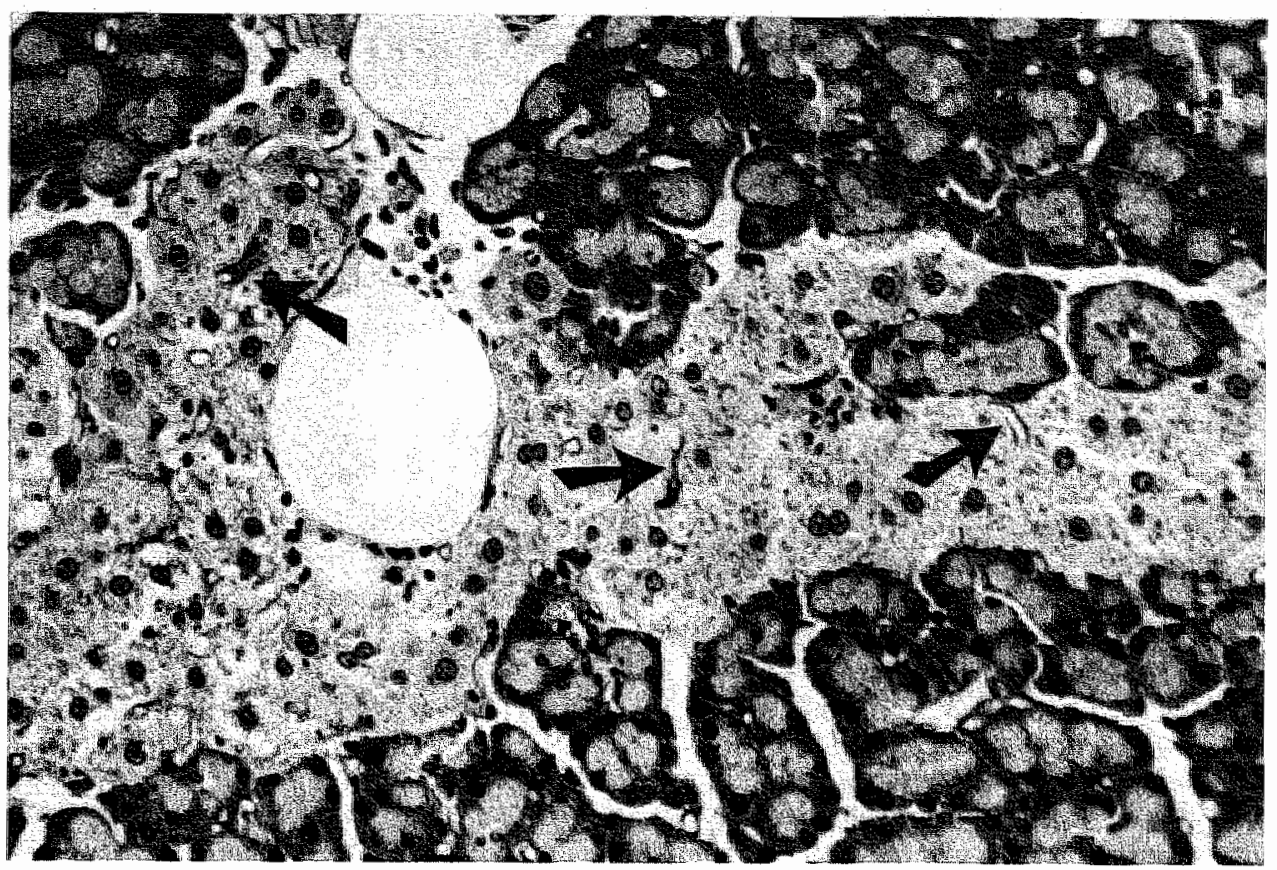

Figure 4. Acinar pancreatic tissue containing a hepatocellular aggregate. Immunocytochemical staining of type IV collagen reveals the basement membranes, that are visible as fine darkstaining structures around capillaries (arrows). Hepatocytes are not lined by a basement membrane (Anti-type IV collagen, x 100).

The assays on pancreatic juice samples revealed extremely small amounts of BMG in two rats of Group I only ( 30 and $60 \mathrm{nM}$, respectively, in absence of BDG). Traces of bilirubin glucuronides were also detected in the digestive juice of two rats of Group II (10 and $260 \mathrm{nM}$ BMG, respectively, with an additional $810 \mathrm{nM}$ BDG in the latter sample). The collections from the duodenum in Groups III and IV did not contain glucuronized bilirubins.

Pre-HTX TB levels (means \pm SEM) in the experimental groups did not differ significantly. Post-HTX formation of bilirubin glucuronides resulted in a small, but statistically significant TB reduction in Group I (from $159 \pm 1.6 \mu \mathrm{M}$ pre-HTX to $141 \pm 1.6 \mu \mathrm{M}$ post-HTX, $\mathrm{p}<0.05$ ), and a more distinct reduction in Group II (from $156 \pm 2.6 \mu \mathrm{M}$ to $132 \pm 1.9 \mu \mathrm{M}, \mathrm{p}<0.01)$. In Groups III and IV, TB levels were not affected 3 months after treatment (pre-HTX TB levels: $156 \pm 2.0 \mu \mathrm{M}$ and $155 \pm 2.3 \mu \mathrm{M}$, respectively, and post-HTX TB levels: $155 \pm 1.6 \mu \mathrm{M}$ and $156 \pm 2.5 \mu \mathrm{M}$, respectively).

\section{Secondary effects of HTX into the pancreas}

The overall state of health in HTX-treated rats was not adversely affected. Mean total body weight of all groups was virtually identical during the experiment. Organ 


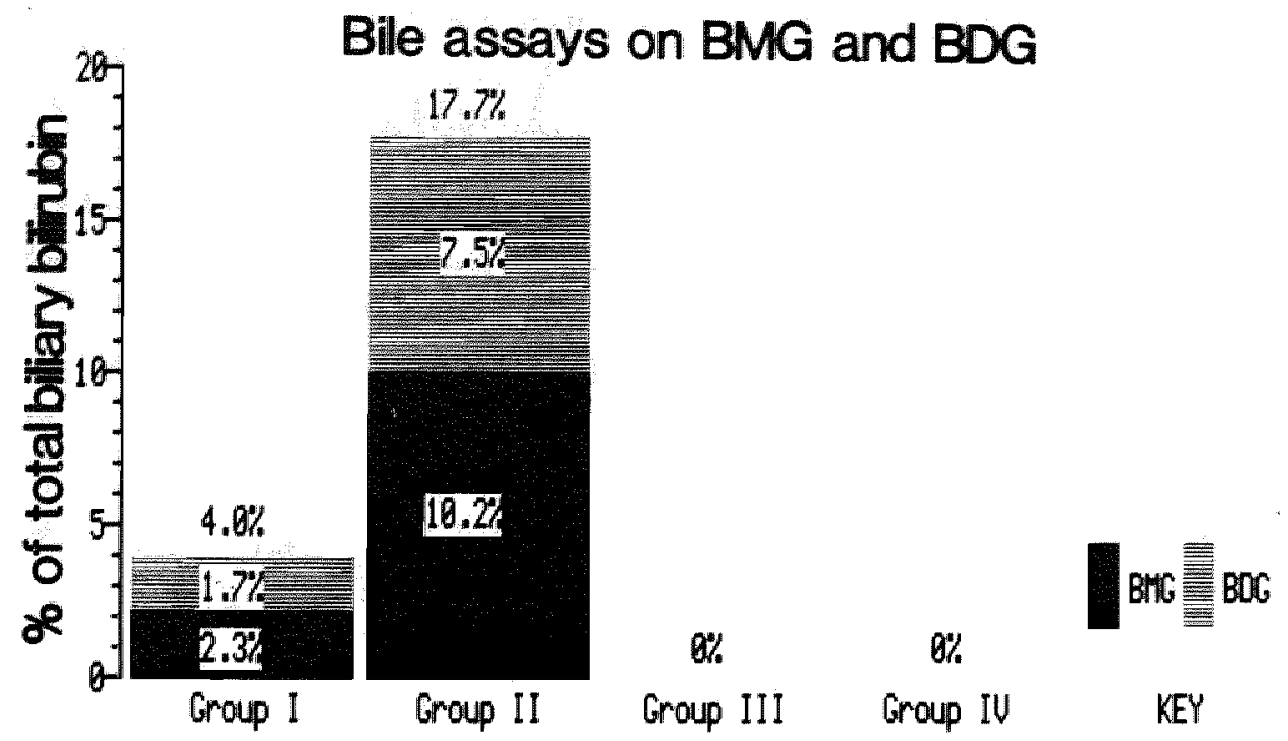

Figure 5. Results of the bile assays on the presence of bilirubin mono-and diglucuronide (BMG and $B D G$, respectively). Mean values are given. The quantity of bilirubin conjugates is expressed as a percentage of the total amount of biliary excreted bilirubin. Bilirubin glucuronides were only found in bile of jaundiced rats that received hepatocytes from normal donors (Groups $I$ and II). The excretion of conjugates was significantly lower in Group I (HTX into the pancreas) than in Group II (HTX into the spleen) $(p<0.002)$. The excretion of BMG exceeded that of $B D G$ in both groups $(p<0.01)$. In Groups III (intrapancreatic HTX with BGT-deficient hepatocytes) and IV (sham-operated rats) bilirubin glucuronides were not excreted.

Table 1. Total body weights and organ weights 3 months after HTX.

\begin{tabular}{llllc}
\hline & $\begin{array}{l}\text { total body } \\
\text { (in grams) }\end{array}$ & $\begin{array}{l}\text { liver } \\
\text { (in mg) }\end{array}$ & $\begin{array}{l}\text { pancreas }^{\mathrm{b}} \\
\text { (in mg) }\end{array}$ & $\begin{array}{l}\text { spleen } \\
\text { (in mg) }\end{array}$ \\
\hline Group I & $304 \pm 9$ & $10,620 \pm 399$ & $1,105 \pm 27$ & $373 \pm 12$ \\
Group II & $312 \pm 12$ & $10,810 \pm 231$ & $1,131 \pm 60$ & $330 \pm 9$ \\
Group III & $305 \pm 10$ & $10,442 \pm 301$ & $984 \pm 34$ & $406 \pm 23$ \\
Group IV & $305 \pm 14$ & $11,083 \pm 350$ & $1,008 \pm 30$ & $392 \pm 14$ \\
\hline
\end{tabular}

a Mean values \pm SEM are given.

- Total body, liver and pancreas weights did not differ significantly between groups.

- Spleen weights in Group II (HTX into the spleen) were significantly lower than in the other groups $(p<0.02)$.

weights at autopsy did not differ significantly between groups, with the exception of a significantly reduced spleen weight in rats that received hepatocytes in the spleen (Group II, p<0.01). Reduction of the spleen weight after HTX into the spleen is a constant finding according to previous research in this field $(5,13)$. The data on body and organ weights are summarized in Table 1. 
Screening for pancreatic disease did not reveal any untoward effects on this organ. Signs of diabetes were not found; glucosuria and weight loss did not occur in any group. The latter finding also ruled out gross disturbances in digestion. Histological signs of pancreatitis were absent as was pointed out earlier. Biochemical evidence of pancreatitis could not be demonstrated either. Mean SA levels did not differ significantly between groups, and were similar to values found in untreated $\mathrm{R} / \mathrm{APfd}-\mathrm{j} / \mathrm{j}$ control rats $(6164 \pm 235 \mathrm{U} / \mathrm{l})$.

\section{Discussion}

This paper is the first report of functional survival of isolated hepatocytes transplanted into the pancreas. It shows that the pancreas provides an appropriate microenvironment for the nidation and survival of the isolated hepatocytes. The presence of so-called hepatotrophic factors in the pancreas possibly plays a role in the substained viability of the grafted liver cells $(19,20)$. Histological examination of the pancreas of rats, in which syngenic or congenic donor hepatocytes were inoculated, showed survival of the transplanted hepatocytes. These cells retained their structural integrity. Cytochemical evidence was provided for sustained glycogen metabolism. As to the locus of nidation of the isolated hepatocytes, there was a marked preference for the interlobular spaces near the vascular structures. Regrouping of the single hepatocytes occurred after transplantation, with formation of hepatic cell cords. This phenomenon was also observed after HTX into the spleen $(4,5,12,13,15)$. Transplanted hepatocytes appeared to have about the same reticulin pattern as in the normal liver. Type IV collagen stains showed that the transplanted hepatocytes, like in the normal situation, lacked basement membranes. Basement membranes, which are located at the interface between epithelial cells and mesenchymal stroma, and connect vascular endothelium with the surrounding matriceal and cellular components of the vessel wall (28), were present in small vascular structures between hepatocytes clustered in the pancreatic tissue. Apparently, a capillary system was being formed in the hepatocellular inoculum. In the periphery of the hepatocyte "islands" degenerative signs were observed. This observation seemed more pronounced in the pancreas of BGT-deficient rats compared to the situation in normal rats. A possible explanation might be the toxic effects of the unconjugated hyperbilirubinemia on the transplanted hepatocytes.

In addition to the histological survival, this study provided substantial proof for the functional viability of isolated normal $R / A P f d$ hepatocytes transplanted into the pancreas of BGT deficient $\mathrm{R} / \mathrm{APfd}-\mathrm{j} / \mathrm{j}$ rats. Because of the total absence of $\mathrm{BGT}$ activity in this rat strain, absolute evidence of metabolic activity of transplanted nondeficient hepatocytes could be obtained by demonstrating the appearance of bilirubin glucuronidation in the deficient recipient. Hence, there could be no doubt of the persisting metabolic activity of the donor hepatocytes in Group I, although the quantity of excreted bilirubin glucuronides (BMG and BDG) was rather small. 
HTX into the spleen proved to be more efficient in the treatment of the enzymatic defect than HTX into the pancreas: bilirubin glucuronide formation and reduction of hyperbilirubinemia were more pronounced in animals that underwent HTX into the spleen. This might be caused by the fact that part of the hepatocyte suspension flows into the portal vein during HTX into the spleen, and allows a subpopulation of donor hepatocytes to nidate and survive in the host liver (5). This "overflow" does not occur during injection into the pancreatic parenchyma. As a result, the total amount of donor hepatocytes that survive in the early post-transplantation period is probably higher in intrasplenic HTX than in intrapancreatic HTX.

Mazzoni et al. (29) performed autotransplantation of liver fragments into the pancreas of the dog, and showed excellent viability of the grafted tissue specimens up to 7 months posttransplantation. He hypothesized that bile duct neoformation might result in bile drainage from the liver fragments into the excretory apparatus of the exocrine pancreas. In order to investigate whether this phenomenon occurred after formation of ectopic hepatic tissue in the pancreas following HTX, we sampled pancreatic juice of the HTX-treated animals. In the animal model used in the present work, unequivocal proof of bile drainage from donor hepatocytes into the pancreatic ducts would have been provided by the demonstration of glucuronized bilirubin in pancreatic secretions. However, evidence of this hypothetical event was not found. Although in two rats of Group I traces of BMG were detected in pancreatic juice, this observation must be ascribed to contamination with remnants of bile in the duodenum, since bilirubin glucuronides were also encountered in the digestive juice collections of two rats of Group II. The possibility seems remote, that the completion of an interconnection between both excretory systems might require more time than the three months we have allowed for in this study.

Negative side-effects of HTX into the pancreas were not observed. The absence of signs of pancreatitis was reassuring, since we feared that pancreatic juice might be activated by bile constituents produced by the grafted liver tissue. The endocrine function of the pancreas did not appear to be adversely affected either, as was shown by the absence of urinary glucose loss or weight loss.

In summary, this work shows that the pancreas is a suitable acceptor organ for the transplantation of isolated hepatocytes. Negative side-effects after HTX into the pancreas were not observed. However, HTX into the spleen appeared to be more effective in treating enzyme deficiency disease than HTX into the pancreas. Bile drainage from the hepatocyte aggregates into the pancreatic excretory system could not be demonstrated.

\section{Acknowledgements}

The authors thank Mrs. B. Engelen (Maastricht), Mrs. C. Leforgeais (Maastricht) and Mr. G. Michiels (Leuven) for their excellent technical assistance and Mrs. K. Spronck (Maastricht) for preparation of the manuscript. Ir. R. Leyten (Proefdieren- 
centrum, University of Lewven, Belgium) kindly provided the animals.

This paper has been accepted for publication in European Surgical Research. Vroemen JPAM, Buurman WA, Van der Linden CJ, Visser R, Heirwegh KPM, Kootstra G.

Transplantation of isolated hepatocytes into the pancreas. European Surgical Research (in press).

\section{References}

1. Matas AJ, Sutherland DER, Steffes MW et al.

Hepatocellular transplantation for metabolic deficiencies: decrease of plasma bilirubin in Gunn rats. Science 1976; 192: 892.

2. Groth $C G$, Arborgh $B$, Björkën $C$, Sundberg $B$, Lundgren $G$.

Correction of hyperbilirubinaemia in the glucuronyl-transferase-deficient rat by intraportal hepatocyte transplantation.

Transplant Proc 1977; 9: 313.

3. Sutherland DER, Matas AJ, Steffes MW, Simmons RL, Najarian JS.

Transplantation of liver cells in an animal model of congenital enzyme deficiency disease: the Gunn rat.

Transplant Proc 1977; 9: 317.

4. Vroemen JPAM, Blanckaert N, Buurman WA, Heirwegh KPM, Kootstra G.

Treatment of enzyme deficiency by hepatocyte transplantation in rats.

J Surg Res 1985; 39: 267.

5. Vroemen JPAM, Buurman WA, Heirwegh KPM, Van der Linden CJ, Kootstra G.

Hepatocyte transplantation for enzyme deficiency disease in congenic rats.

Transplantation 1986; $42: 130$.

6. Sutherland DER, Numata M, Matas AJ, Simmons RL, Najarian JS.

Hepatocellular transplantation in acute liver failure.

Surgery $1977 ; 82: 124$.

7. Cuervas-Mons V, Cienfuegos JA, Maganto $P$ et al.

Time-related efficacy of liver cell isografts in fulminant hepatic failure.

Transplantation 1984; 38: 23.

8. Minato M, Houssin D, Demma I et. al.

Transplantation of hepatocytes for treatment of surgically induced hepatic failure in the rai.

Eur Surg Res 1984; 16: 162.

9. Makowka L, Falk RE, Rotstein LE et al.

Cellular transplantation in the treatment of experimental hepatic failure.

Science 1980; 210: 901.

10. Makowka $\mathbb{L}$, Rotstein LE, Falk RE et al.

Reversal of toxic and anoxic induced hepatic failure by syngeneic, allogeneic and xenogeneic hepatocyte transplantation.

Surgery 1980; 88: 244.

11. Makowka L, Rotstein LE, Falk RE et al.

Allogeneic and xenogeneic hepatocyte transplantation in experimental hepatic failure.

Transplaniation 1980; 30: 429 .

12. Mito M, Ebata H, Kusano M, Onishi T, Hiratsaka M, Saito T.

Studies on ectopic liver utilizing hepatocyte transplantation into the rat spleen.

Transplant Proc 1979; 11: 585.

13. Mito M, Ebata H, Kusano M, Onishi T, Saito T, Sakamoto S.

Morphology and function of isolated hepatocytes Iransplanted into rat spleen.

Transplantation 1979; $28: 499$. 
14. Kusano $M$, Mito $M$.

Observations on the fine structure of long-survived isolated hepatocytes inoculated into rat spleen. Gastroenterology $1982 ; 82: 616$.

15. Vroemen JPAM, Van der Linden CJ, Butrman WA, Coemegracht J, Heirwegh KPM, Kootstra G. In wivo dynamic $\%$ m $T$-HIDA scintigraphy after hepatocyte transplantation: a new method for the monitoring of graft function.

Eur Surg Res 1987; 19: 140.

16. Selden C, Gupta S, Johnstone R, Hodgson HJF.

The pulmonary wascular bed as a site for implantation of isolated liver cells in inbred rats.

Transplantation 1984; 38: 81.

17. Demetriou $A A$, Whiting JF, Feldman $D$ et al.

Replacement of liver function in rats by transplantation of microcarrier-attached hepatocytes. Science $1986 ; 233: 1190$.

18. Demetriou AA, Whiting J, Levenson $\mathrm{SM}$ et al.

New method of hepatocyte transplantation and extracorporeal liver support.

Ann Surg 1986; 204: 259 .

19. Kirsch RE, Terblanche J.

Liwer injury and regeneration.

In: The liver, a series of critical surveys of the international literature. Edited by: Arias IM, Frenkel M, Wilson JHP. Excerpta Medica, Amsterdam 1982.

20. Starzl TE, Porter KA, Francavilla JA, Benichou J, Putnam CW.

A hundred years of the hepatotrophic controversy.

In: Hepatotrophic Factors. Edited by: Porter R, Whelan J. Elsevier/Excerpta Medica/NorthHolland, Amsterdlam 1978.

21. Leyten R, Vroemen JPAM, Blanckaert N, Heirwegh KPM.

The congenic normal R/APfd and jaundiced R/APfd-j/j rat strains: a new animal model of hereditary non-haemolytic unconjugated hyperbilirubinaemia due to defective bilirubin conjugation. Laboratory Animals 1986; $20: 335$.

22. Scragg I, Celier C, Burchell B.

Congenital jaundice in rats due to the absence of hepatic bilirubin UDP-glucuronyltransferase enzyme protein.

FEBS Letters 1985; 183: 37.

23. Seglen PO.

Preparation of isolated rat liver cells.

Methods Cell Biol 1976; 13: 29.

24. Muraca M, Blanckaert N.

Liquid-chromatographic assay and identification of mono- and diester conjugates of bilirubin in normal serum.

Clin Chem 1983; 29: 1767.

25. Henkel E, Morich S, Henkel $R$.

2-Chloro-4-nitrophenyl-beta-D-maltoheptaoside: a new substrate for the determination of alphaanylase in serum and urine.

J Clin Chem Clin Biochen 1984; 22: 489.

26. Visser R, Van der Beek JMH, Havenith MG, Cleutjens JPM, Bosman FT.

Immunocytochemical detection of basement membrane antigens in the histopathological evaluation of laryngeal dysplasia and neoplasia.

Histopathology 1986; $10: 171$.

27. Bruning JL, Kintz BL.

Computational handbook of statistics. 2nd edition.

Scott, Foresman and Company. Glenview, Illinois 1977.

28. Martinez-Hernandez A, Amenta PS.

The basement membrane in pathology.

Laboratory Investigation $1983 ; 48,656$.

29. Mazzoni G, Di Martino C, Scarpelli F, Cristini F, Citarella G, Martini ME.

Liver autotransplantation into the pancreas.

Transplantation 1982; 34: 108 . 


\section{The cytokinetic behaviour of donor hepatocytes after syngenic hepatocyte transplantation into the spleen}

\section{Summary}

The cytokinetic behaviour of isolated hepatocytes transplanted into the spleen of syngenic normal Wistar rats was studied. Hepatocyte transplantation was performed by the intrasplenic injection of $10^{7}$ isolated hepatocytes. The proliferation index (PI) of intrasplenic donor hepatocytes was assessed by immunocytochemical visualization of DNA-synthesizing cells after pulse labeling with bromodeoxyuridine (BrdU), a thymidine analogue. A method was developed for determination of intrasplenic liver mass by means of a method based on hepatocellular GLDH content. The spontaneous PI of donor hepatocytes at 12 and at 20 weeks post-HTX amounted to around 3\%. A significant increase of intrasplenic liver mass was demonstrated between the 12th and 20th week post-HTX (from $8.1 \pm 0.8 \%$ to $10.8 \pm 0.8 \%$ of spleen weight, $\mathrm{p}<0.05$ ). After partial hepatectomy ( $\mathrm{PH})$ at 12 weeks post-HTX, the PI of liver cells in the spleen showed a transient increase up to about $10 \%$, which rapidly declined to the "spontaneous"' level of 3\%. However, PH did not cause an additional increase in intrasplenic liver mass.

This study shows that continuous mitotic activity of intrasplenic hepatocytes results in an actual increase of liver mass in spleen. Although modulation of proliferative activity of ectopically grafted hepatocytes was shown to occur after PH in the HTXtreated rat, this procedure did not result in an additional increase of intrasplenic liver tissue. 


\section{Introduction}

The promising results of experimental hepatocyte transplantation (HTX) in the treatment of enzyme deficiency disease (1-6) and severe liver failure (7-11) necessitate further research into this transplantation technique. The spleen is an appropriate acceptor organ for isolated hepatocytes $(4,5,11-15)$. Long-term functional survival of hepatocytes transplanted into the spleen has been well documented, but there is still debate concerning the spontaneous cytokinetic behaviour after nidation $(5,12-17)$. This aspect of HTX needs further evaluation, since the efficacy of metabolic support provided by HTX will be proportional to the resulting donor liver cell mass. For the same reason, the influence of a potent stimulus for liver cell proliferation, such as partial hepatectomy $(\mathrm{PH})$, on the transplanted hepatocyte population is also of interest.

In this study we investigated the cytokinetic behaviour of liver cells grafted in the spleen. A non-autoradiographic method for visualization of cells in the DNAsynthesizing phase (S-phase) of the cell cycle was applied to study the proliferative activity of hepatocytes transplanted into the spleen. A method based on cellular enzyme content was developed for quantification of donor liver mass in the spleen. By means of this method relative and absolute quantities of hepatic tissue in the spleen were compared at 12 and 20 weeks post-HTX. In addition, the effects of partial hepatectomy in the host on proliferation rate of grafted hepatocytes and on intrasplenic liver tissue mass were studied.

\section{Materials and methods}

\section{Animals}

Adult male rats of the inbred Wistar R/APfd strain (275-350 g, purchased from the Proefdierencentrum, University of Leuven, Leuven, Belgium), were used as hepatocyte donors and recipients. All animals had free access to standard laboratory chow and water. All surgical procedures were performed under ether anesthesia.

\section{Hepatocyte transplantation}

A detailed description of our technique of HTX has been given in previous reports $(4,5)$. In brief, isolation of hepatocytes was performed with the two-step collagenase digestive method as described by Seglen (18). Cell viability, assessed by the trypan blue exclusion test, ranged from $85-95 \%$. A cell suspension containing $10^{7}$ viable hepatocytes per $\mathrm{ml}$ was prepared and stored on ice. One $\mathrm{ml}$ of this cell supension was slowly injected into the splenic parenchyma of the recipient rat. 


\section{Experimental design}

A first series of experiments was designed a) to determine proliferative activity of donor hepatocytes at various intervals after nidation in the spleen and b) to evaluate the effect of partial hepatectomy on the proliferative behaviour of these cells.

Twenty R/APfd rats underwent HTX. Eight of these rats did not undergo further treatment. These rats served to study the spontaneous proliferation of intrasplenic hepatocytes in HTX-treated animals. At 12 and 20 weeks post-HTX, 4 rats were sacrificed one hour after administration of Bromodeoxyuridine (BrdU), a labeling agent for DNA-synthesizing cells. Twelve rats were subjected to partial hepatectomy $(\mathrm{PH})$ at 12 weeks post-HTX, in order to study alterations in proliferative activity induced by $\mathrm{PH}$. They were sacrificed on day $2,4,7$ and 10 post-PH (each time $\mathrm{n}=2$ ) and at 8 weeks post-PH $(n=4)$, one hour after injection with BrdU. Liver and spleen of all animals were weighed and fixed for histological processing. In this material the proliferative activity of hepatocytes was studied.

A second series of experiments was set up a) to study the evolution of donor liver mass in the spleen in relation to the duration of the posttransplant period, and b) to determine both short- and long-term effect of $\mathrm{PH}$ on intrasplenic liver tissue mass. For this purpose, a method was developed to quantify liver tissue mass present in splenic tissue. This was achieved by assaying the content of glutamate dehydrogenase (GLDH) of HTX-treated spleens.

Twenty-six R/APfd rats underwent HTX, and were randomly divided in Group I $(n=12)$ and Group II $(n=14)$. At twelve weeks post-HTX all rats of Group II underwent $\mathrm{PH}$, whereas rats of Group I underwent sham-PH (laparotomy with gentle manipulation of the liver). Three days post-PH, 6 rats of Group I (Group I-A) and 7 rats of Group II (Group II-A) were sacrificed. Minute biopsies were taken from the spleen to verify graft survival. Body weight, spleen and liver weights were recorded. Homogenates of the entire spleen of each animal were prepared for enzyme assays, in order to assess the amount of liver mass contained in the spleen. The remaining 6 rats of Group I (Group I-B) and 7 rats of Group II (Group II-B) were sacrificed at 20 weeks post-HTX (i.e. 8 weeks after laparotomy and PH, respectively). On these subgroups identical investigations were executed as on subgroups I-A and II-A. GLDH contents in the spleens were determined. Relative and absolute quantities of intrasplenic liver tissue mass were computed, and compared between groups.

\section{Partial hepatectomy (PH)}

A $70 \% \mathrm{PH}$ was performed as described by Waynforth (19). This technique implies the resection of the middle and left lateral lobes of the liver, leaving a hepatic remnant of approximately $30 \%$ of the original liver weight. All animals survived the PH and sham-PH procedure. 


\section{Detection of transplanted hepatocytes in S-phase}

Proliferating cells can be pulse labeled with Bromodeoxyuridine (BrdU), a thymidine analogue. This molecule is incorporated into DNA of cells, which are in the $S$-phase of the cell cycle (20). An anti-BrdU monoclonal antibody is subsequently used for the immunocytochemical detection of BrdU-labeled cells. This procedure provides a non-autoradiographic method for direct visualization of DNA synthesizing cells in tissue sections, which permits the exact localization of proliferating cells, and also allows the analysis of the cytokinetics of phenotypically defined cells in heterogeneous populations (21). We used this technique for the investigation of proliferative behaviour of transplanted hepatocytes.

In practice, HTX-treated rats were injected intravenously with $50 \mathrm{mg} / \mathrm{kg}$ BrdU. One hour was allowed for the incorporation of BrdU in S-phase cells. After one hour the rats were sacrificed and spleen, liver and a segment of small intestine were removed for histological processing. Immunocytochemical staining of small intestine was performed as a control stain of BrdU-labeled proliferative intestinal mucosa cells in the crypts of Lieberkuehn.

As parameter for the proliferative activity of the recipients hepatocytes and of the intrasplenic donor hepatocytes, the proliferative index (PI) of these cells was calculated as the ratio (given in percentage) of the number of BrdU-labeled hepatocytes to the total number of counted hepatocytes. In each HTX-treated spleen a minimum of 500 transplanted hepatocytes was counted for determination of the PI.

\section{Histological processing}

Tissues were fixed in $10 \%$ buffered formaldehyde and embedded in paraplast. Immunocytochemical BrdU and keratin staining procedures, as well as hematoxylin \& eosin (H\&E) and periodic acid Schiff (PAS) routine stains were carried out on $2-\mu \mathrm{m}$ sections.

For the immunocytochemical detection of DNA-incorporated BrdU the sections were mounted on gelatin-coated slides, dried overnight, and incubated in $2 \mathrm{~N} \mathrm{HCl}$ for 30 minutes at $37^{\circ} \mathrm{C}$, thus unmasking the BrdU antigenic sites by acid denaturation of DNA. After treatment with $0.1 \mathrm{M}$ sodiumtetraborate $\mathrm{pH} 8.5$, slides were washed in phosphate buffered saline (PBS) and incubated with monoclonal anti$\mathrm{BrdU}$ antibody for one hour at $37^{\circ} \mathrm{C}$. Anti-BrdU monoclonal antibody was produced as described by Schutte et al. (21). As second step reagent peroxidase conjugated rabbit antimouse $\lg$ was used. Visualization of the immunoreactive sites was achieved by applying $0.04 \% 3,3^{\prime}$-diaminobenzidinetetrahydrochloride in $0.05 \mathrm{M}$ Tris $\mathrm{pH} 7.6$ to which $0.01 \% \mathrm{H}_{2} \mathrm{O}_{2}$ was added immediately before use. For optimal interpretation of the histology, the sections were counterstained with $\mathrm{H} \& \mathrm{E}$. 

animals

In search of a reliable method for measuring liver tissue mass present in splenic tissue by means of enzyme assays, we performed pilot enzyme assays of lactate dehydrogenase (LDH), aspartate transaminase (AST), glutamate dehydrogenase (GLDH), alkaline phosphatase (AF), gamma-glutamyl transpeptidase (gamma-GT), creatin kinase (CK), and alanine transaminase (ALT) on spleen and liver homogenates and on mixed homogenates of these tissues. The enzyme activities in tissue homogenates were assayed spectrophotometrically at $25^{\circ} \mathrm{C}$ using commercially available test kits (Boehringer, Mannheim, FRG). Homogenates were prepared of $500 \mathrm{mg}$ liver or $500 \mathrm{mg}$ splenic tissue, in a volume of $10 \mathrm{ml}$ PBS. Of these homogenates we composed mixed homogenates with a liver/spleen ratio of $1 \%$, $5 \%, 10 \%, 25 \%, 50 \%$ and $75 \%$. Of all the enzymes studied in the homogenates, the concentrations of GLDH correlated best with the liver/spleen ratio (see Results). This correlation provided the basis for computation of relative and absolute liver tissue mass present in the HTX-treated spleen.

Tissue homogenates of the entire spleen of each HTX-treated rat (minus a minute biopsy for the demonstration of graft survival) were prepared in a volume of $10 \mathrm{ml}$ PBS according to standard laboratory techniques. The concentration of GLDH in each homogenate was measured, and the amount of GLDH released from the homogenized tissue was expressed in Units GLDH per gram of homogenized tissue. From the resulting values (in $\mathrm{U} / \mathrm{g}$ ), relative and absolute amounts of liver tissue mass in the spleen of each animal were computed. Mean values of each group were compared.

\section{Statistical analysis}

For comparison between groups the t-test for a difference between two independent means was used.

\section{Results}

\section{Spontaneous proliferative activity of intrasplenic donor hepatocytes}

The survival of donor hepatocytes in the spleen was demonstrated in all rats subjected to syngenic HTX. Aggregates of morphologically intact hepatocytes, ranging in number from a few to more than several thousand, were found in the red pulp of the spleen (Figure 1). These aggregates were not always evenly distributed throughout the spleen. Although the formation of hepatic cords was observed, reconfiguration of the typical liver structure did not occur. Many of the transplanted cells had a binuclear appearance. PAS stains revealed the persisting capacity of these cells of storing glycogen. Mitotic figures of transplanted hepatocytes could not be dis- 


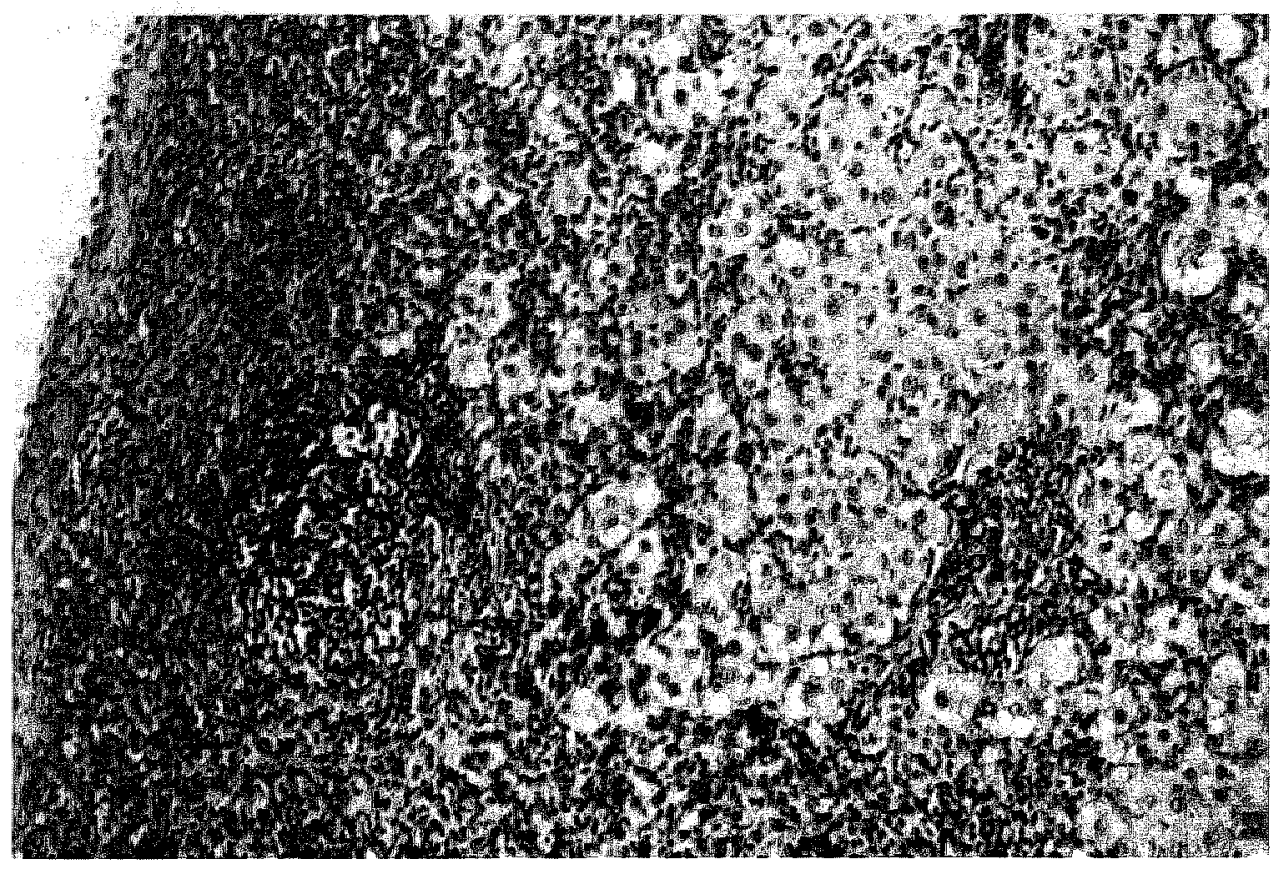

Figure 1.

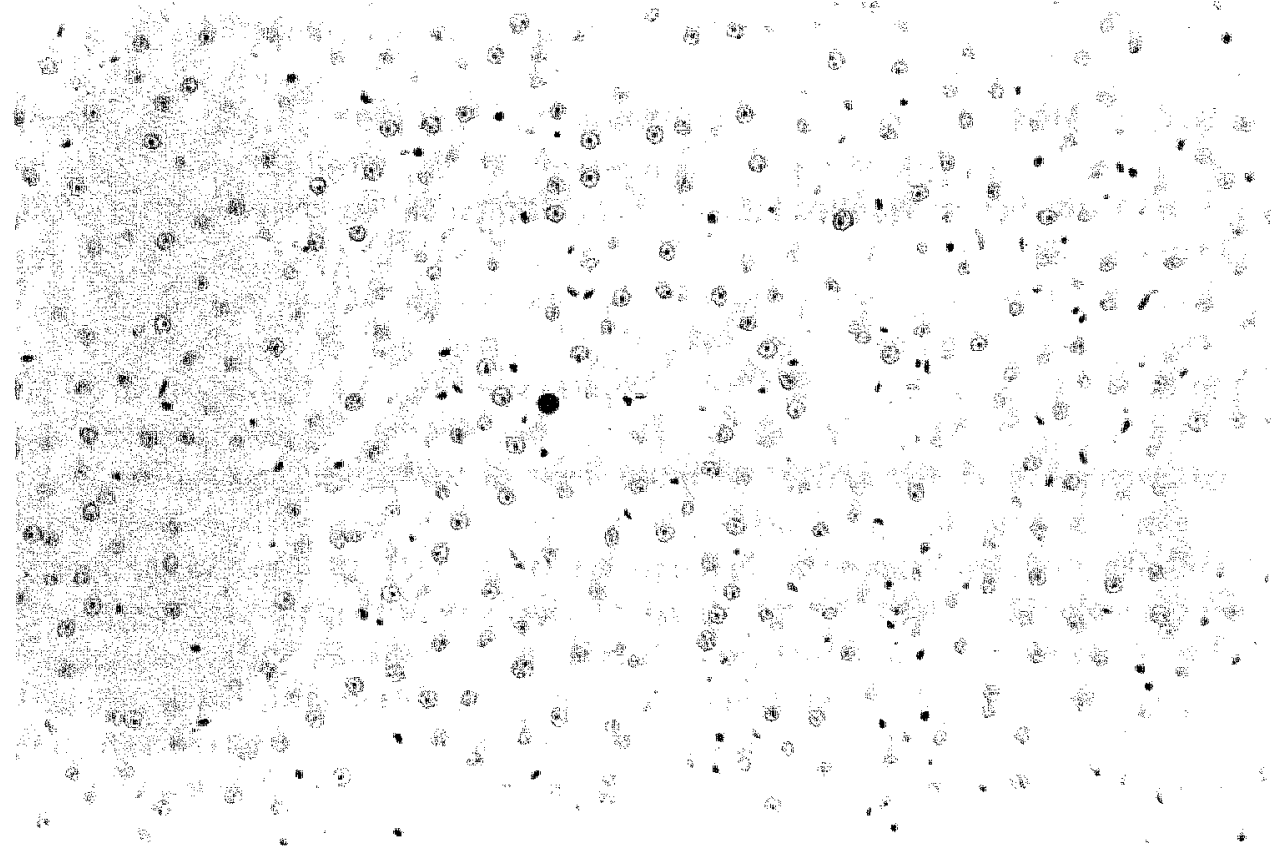

Figure 2A. 
Figure 1. Syngenic hepatocytes in the hast spleen 12 weeks after transplantation. Hepatocellular aggregates are found in the red pulp. Hepatocytes show normal histological features, although in some areas degenerative changes are abserved. (H\&E, $\times 50)$.

Figure 2.4. Immunocytochemical visualization of DNA-synthesizing cells after pulse labeling with BraU. Appearance of the "quiescent" liver of a HTX-treated rat at 12 weeks post-HTX. Less than I per five thousand hepatocytes are BrdU-positive. (BrdU stain, $\times 75)$.

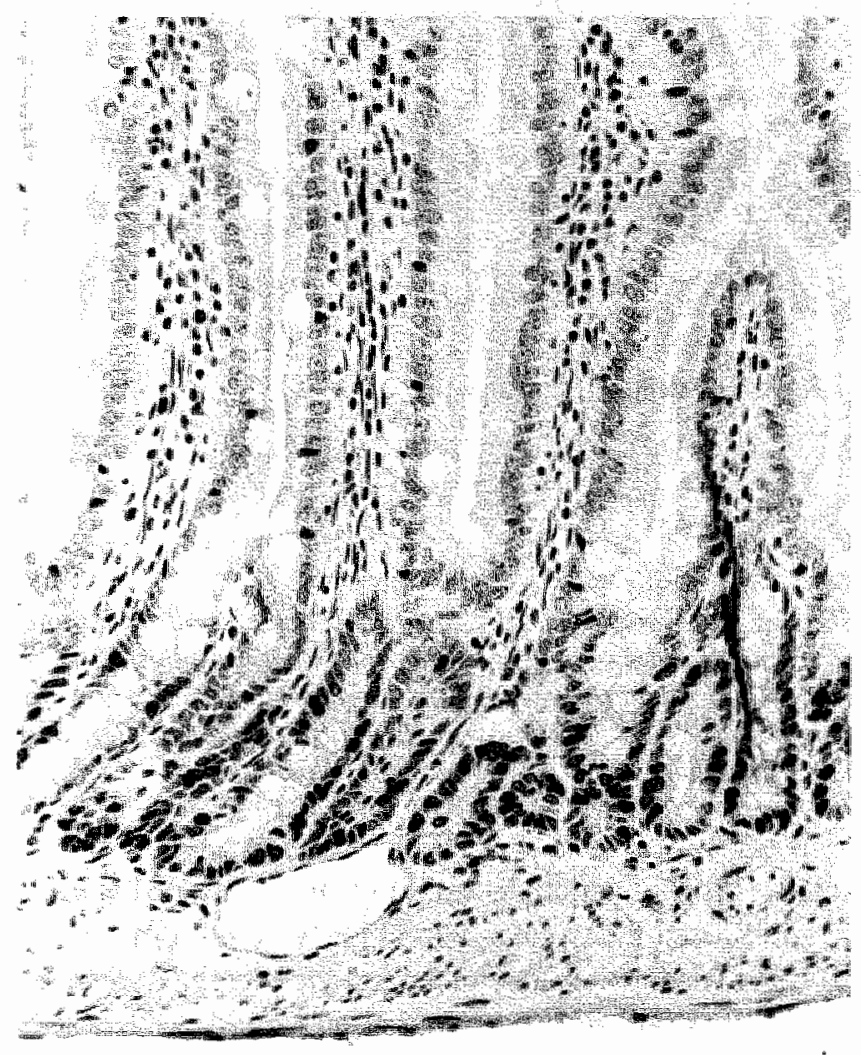

Figure 2B. Control BrdU stain of small intestine of the same rat as in Figure $2 A$. The dark staining proliferating DNA-synthesizing cells at the base of the crypts can easily be distinguished from the non-proliferating cells. (BrdU stain, $x$ 100).

cerned. Histochemical staining of keratin added to a positive identification of intrasplenic liver cells (not shown). In some areas degenerative alterations of the heptocytes were observed, such as cytoplasmatic vacuoles, fatty infiltration or cell shrinkage. These findings were in accordance with previous observations (5). Differ- 


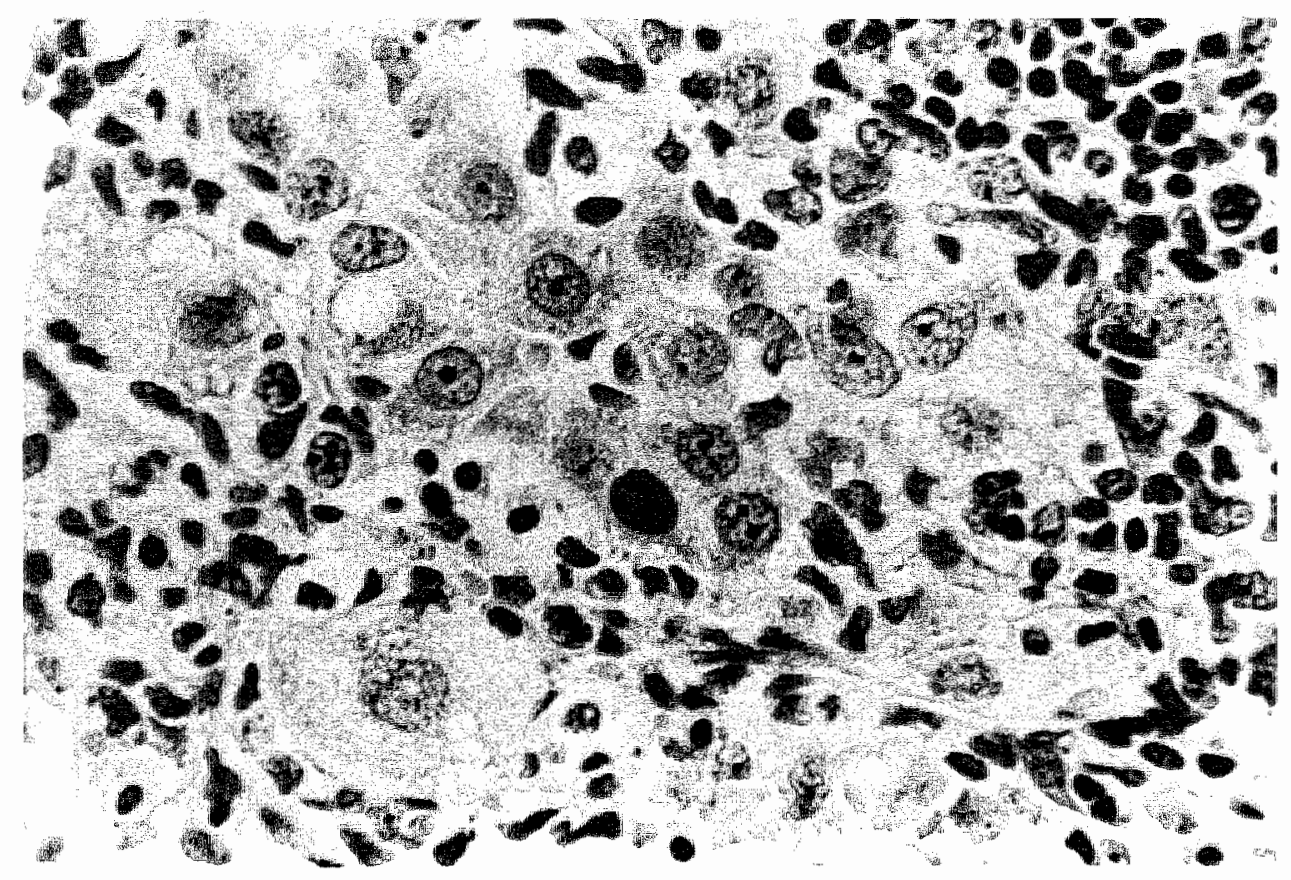

Figure 2C. Donor hepatocytes in the spleen at 12 weeks post-HTX. BrdU staining demonstrates the presence of spontaneous proliferative activity in the donor cell population. The large, dark staining BrdU-positive nuclei of hepatocytes in S-phase are well distinct from the pale nuclei of the non-proliferating hepatocytes. This allows calculation of the proliferation index in the hepatocellular transplamt, which was $3.6 \%$ in this rat. (BrdU stain, $x$ 250).

ences in histological features, on the basis of which distinction could be made between spleens of 12 weeks post-HTX and those of 20 weeks post-HTX were not present.

Spontaneous proliferative activity of syngenic donor hepatocytes in the spleen of HTX-treated normal Wistar (R/APfd) rats was investigated at 12 and at 20 weeks post-transplantation. In histological preparations of the host liver virtually none of the hepatocytes (less than 1 per five thousand in all animals studied) exhibited nuclear BrdU incorporation (Figure 2A). Consequently, the PI in the liver of these animals was $0 \%$. Control BrdU stains of small intestine clearly showed the uptake of BrdU in the germinative cells at the base of the crypts of Lieberkuehn (Figure 2B). In the spleen, the grafted liver cells showed manifest signs of proliferative activity. BrdU incorporation in nuclear DNA by these ectopic donor hepatocytes could be demonstrated in all rats (Figure $2 \mathrm{C}$ ). Liver cells with BrdU-positive nuclei were randomly distributed over the intrasplenic hepatocyte population. The PI of transplanted liver cells ranged from 2.8 to $3.8 \%$ (mean value: $3.4 \%$ ) at 12 weeks post-HTX and from 2.4 to $3.9 \%$ (mean value: $3.3 \%$ ) at 20 weeks post-HTX. These data are presented in Table 1 , in addition to liver and spleen weights of the HTXtreated rat studied. 
Table 1 . Weights of liver and spleen of HTX-treafed rats and the proliferation index (Pl) of hepatocyles in these organs. Measurements at 12 and 20 weeks post-HTK.

\begin{tabular}{|c|c|c|c|c|c|c|}
\hline \multirow{2}{*}{ Group } & \multirow{2}{*}{$\begin{array}{l}\text { weeks } \\
\text { post-HTX }\end{array}$} & \multirow[t]{2}{*}{ rat } & \multicolumn{2}{|l|}{ liver } & \multicolumn{2}{|l|}{ spleen } \\
\hline & & & $\begin{array}{l}\text { weight } \\
\text { (in mg) }\end{array}$ & PI & $\begin{array}{l}\text { weight } \\
\text { (in mg) }\end{array}$ & PI \\
\hline & & 1 & 15,670 & $0 \%$ & 452 & $3.0 \%$ \\
\hline & 12 weeks & 2 & 14,890 & $0 \%$ & 473 & $3.8 \%$ \\
\hline & posit-HTX & 3 & 15,110 & $0 \%$ & 458 & $2.8 \%$ \\
\hline & & 4 & 14,400 & $0 \%$ & 414 & $3.6 \%$ \\
\hline \multicolumn{7}{|c|}{ HTX only } \\
\hline & & 5 & 14,230 & $0 \%$ & 440 & $3.9 \%$ \\
\hline & 20 weeks & 6 & 15,070 & $0 \%$ & 369 & $2.4 \%$ \\
\hline & post-HTX & 7 & 14,950 & $0 \%$ & 4116 & $3.4 \%$ \\
\hline & & 8 & 15,260 & $0 \%$ & 378 & $3.0 \%$ \\
\hline
\end{tabular}

The influence of partial hepatectomy on proliferative behaviour of intrasplenic hepatocytes

Compensatory hyperplasia of the remaining liver lobes occurred in the 12 HTXtreated animals that underwent PH 12 weeks after receiving the hepatocellular transplant. Regeneration of the liver following the $70 \%$ surgical removal of this organ was completed after 10 days. Weights of the regenerating liver and spleen at the various intervals after PH and the associated PI levels are listed in Table 2. The PI of the liver reached a peak on day 2 post-PH (17.8 and $13.2 \%$, Figure $3 \mathrm{~A})$, and decreased thereafter. On day 7 less than 1 per thousand hepatocytes appeared to be BrdU-positive. On day 10 a new, albeit small, increase of the PI was observed in the liver (2.0 and $1.5 \%$ ). Eight weeks after partial liver removal, PI in the liver was identical to the PI observed in the untouched liver, i.e. $0 \%$.

The donor hepatocytes in the spleen clearly showed an increase of the PI after partial liver removal. The maximum BrdU uptake was recorded at day 2, when the PI of intrasplenic hepatocytes amounted to 11.0 and $8.4 \%$ in the rats studied (Figure 3B). On day 4 these figures were 5.4 and $3.8 \%$. In the later postoperative period (on day 7, on day 10 and at 8 weeks after PH) PI of hepatocytes in the spleen remained at the level of spontaneous proliferative activity as was observed in HTXtreated rats that underwent HTX only (around 3\%).

Quantitative assessment of liver tissue mass contained in the spleen of HTX-treated rats

A method for quantification of intrasplenic liver tissue mass was developed by enzyme assays in spleen, liver and mixed liver/spleen homogenates. Of the enzymes studied, GLDH was found to be the most suitable enzyme for this purpose. This 
Table 2. Weights of liver and spleen of HTX-treated rats that underwent partial hepatectomy (PH) at 12 weeks post-HTX and the proliferation index (PI) of hepatocytes in these organs. Measurements at 12 weets and 20 weeks post-HTX.

\begin{tabular}{|c|c|c|c|c|c|c|c|}
\hline \multirow{2}{*}{ Growp } & \multirow{2}{*}{$\begin{array}{l}\text { weeks } \\
\text { post-HTX }\end{array}$} & \multirow{2}{*}{$\begin{array}{l}\text { days } \\
\text { post-PH }\end{array}$} & \multirow[t]{2}{*}{ rat } & \multicolumn{2}{|l|}{ liver } & \multicolumn{2}{|l|}{ spleen } \\
\hline & & & & $\begin{array}{l}\text { weight } \\
\text { (in } \mathrm{mg} \text { ) }\end{array}$ & PI & $\begin{array}{l}\text { weight } \\
\text { (in } \mathrm{mg} \text { ) }\end{array}$ & PI \\
\hline & & 2 & 1 & 6,150 & $17.8 \%$ & 436 & $11.0 \%$ \\
\hline & & 2 & 2 & 8,420 & $13.2^{0 \%}$ & 45.1 & $8.4 \%$ \\
\hline & & 4 & 3 & 12,150 & $6.40 \%$ & 433 & $5.40 \%$ \\
\hline & 12 weeks & 4 & 4 & $\mathbb{1 1}, 350$ & $4.2 \%$ & 450 & $3.8 \%$ \\
\hline & post-HTX & 7 & 5 & 14,790 & $0.10 \%$ & 475 & $2.5 \%$ \\
\hline & & 7 & 6 & 15,680 & $0.110 \%$ & 427 & $3.0 \%$ \\
\hline HTX & & 10 & 7 & 15,400 & $2.0 \% 0$ & 460 & $2.6 \%$ \\
\hline \multirow[t]{5}{*}{$+\mathrm{PH}$} & & 10 & 8 & 14,680 & $1.5 \%$ & 430 & $4.4 \%$ \\
\hline & & 55 & 9 & 13,280 & 0 of & 368 & $2.7 \%$ \\
\hline & 20 weeks & 55 & 10 & 13,590 & $0 \%$ & 406 & $3.7 \%$ \\
\hline & post-HTX & 55 & 11 & 14,390 & $0 \%$ & 375 & $3.1 \%$ \\
\hline & & 55 & 12 & 14,660 & $0 \%$ & 448 & $2.9 \%$ \\
\hline
\end{tabular}

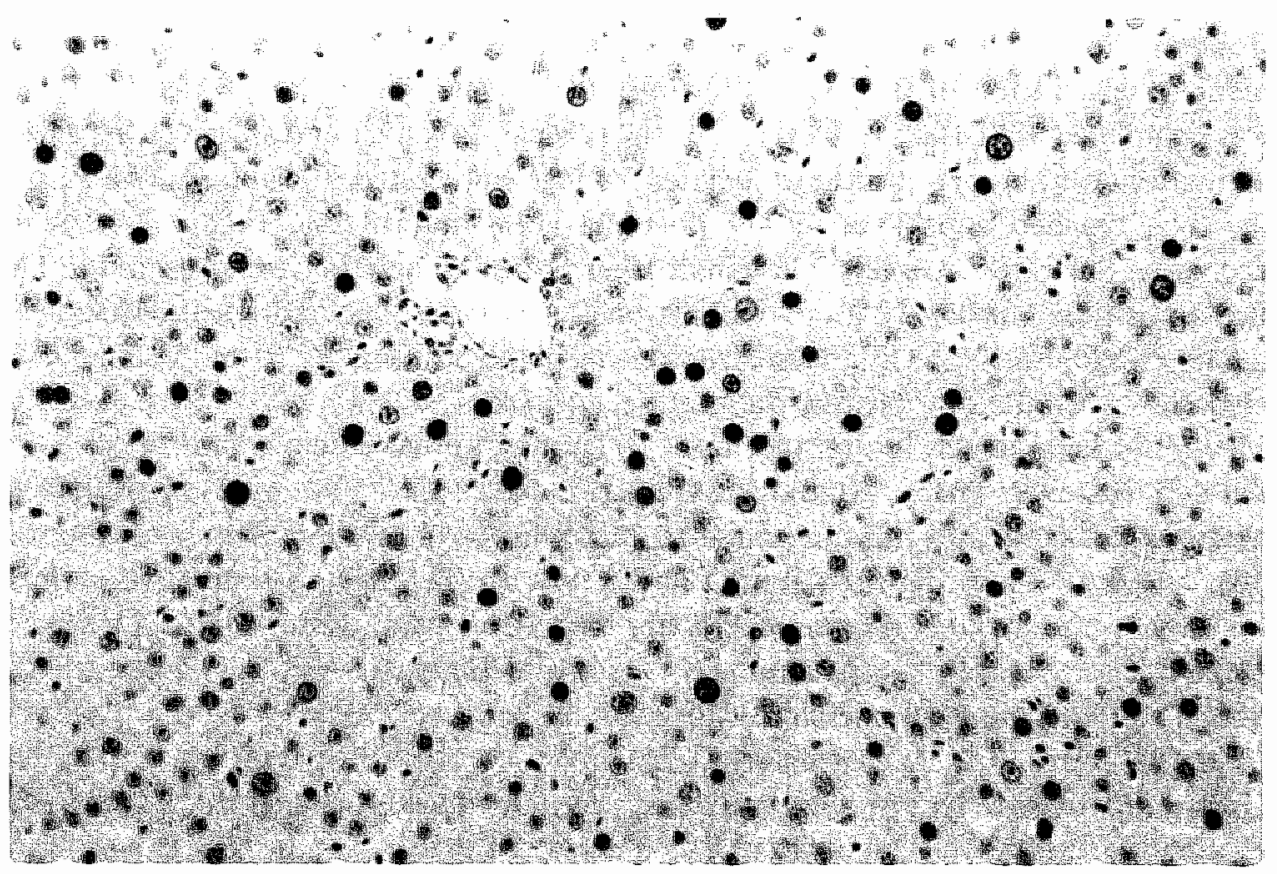

Figure 3A. BrdU stain of the regenerating liver at the second day after PH. An enormows increase in proliferative activity is observed compared to the situation before $P H$ as shown in Figure 2A. In the hepatic remnant of this HTX-treated rat a PI of $17.8 \%$ was found. (BrdU stain, $x 75$ ). 


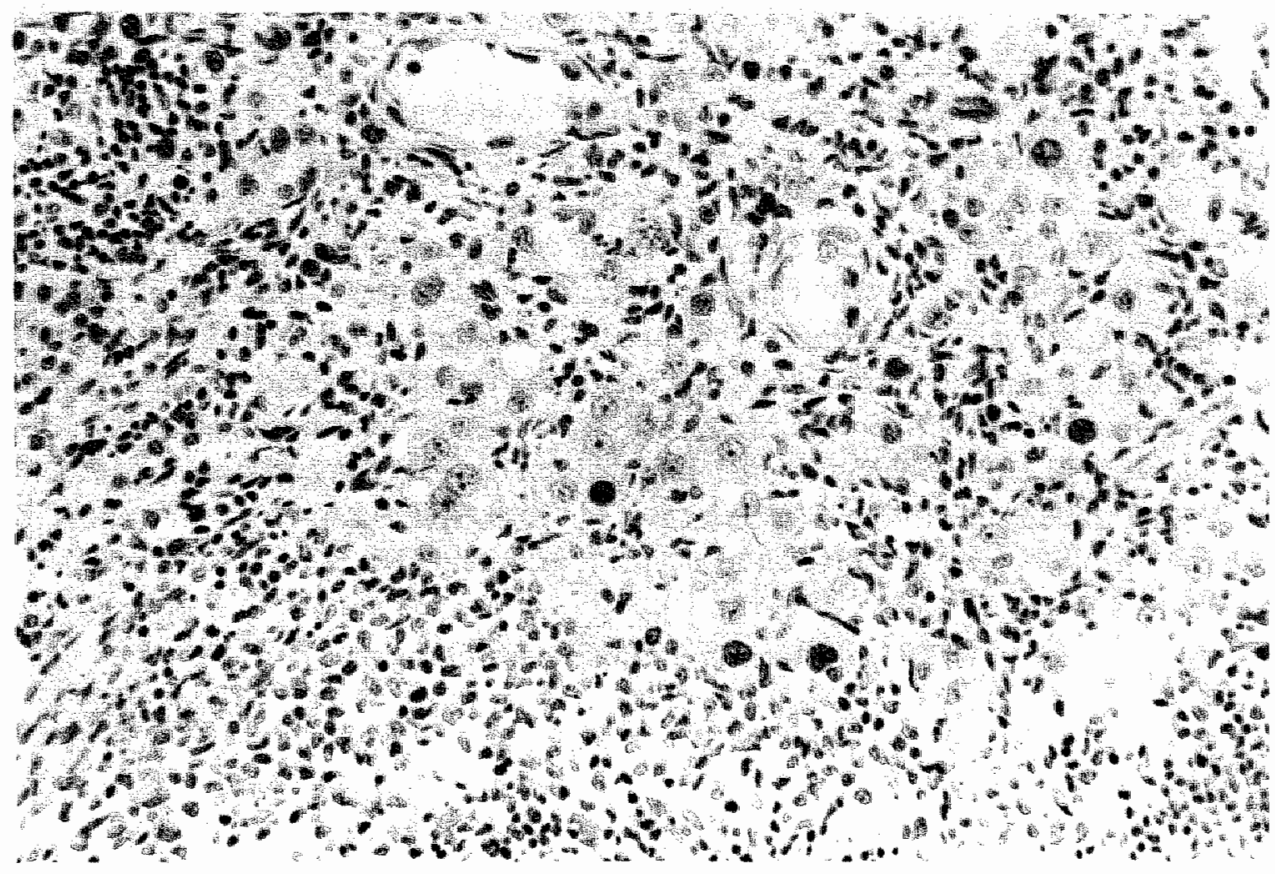

Figure 3B. Donor hepatocytes in the spleen of the same rat as in Figure $3 A$. At the second day after PH, an increase in the proportion of BrdU-labeled hepatocytes was observed. (PH was performed at the $12 \mathrm{th}$ week post-HTX). The PI of the intrasplenic hepatocytes amounted to $11.0 \%$. (BrdU $\operatorname{stain}, \times 100)$.

enzyme was present in constantly low amounts in spleen (mean \pm SEM: $8.8 \pm 0.35$ $\mathrm{U} / \mathrm{g}$ tissue, mean of 9 measurements) and in constant and relatively high amounts in liver $(183.5 \pm 2.5 \mathrm{U} / \mathrm{g}$ tissue, mean of 9 measurements). GLDH content of regenerating liver (i.e. after PH) was not different from GLDH content measured in normal liver (data not given). Theoretically, the proportional and hence the absolute amount of liver tissue contained in a spleen could now be calculated. Supposing a HTX-treated spleen, that contains m(easured) $\mathrm{U} / \mathrm{g}$ GLDH, as consisting of a $\%$ splenic tissue (containing $8.8 \mathrm{U} / \mathrm{g} \mathrm{GLDH}$ ) and b \% hatic tisue (containing 183.5 $\mathrm{U} / \mathrm{g} \mathrm{GLDH}$ ), then the following equations are valid:

$$
\mathrm{a} \%+\mathrm{b} \%=100 \% \text { or } \mathrm{a} / 100+\mathrm{b} / 100=1
$$

and

$$
\mathrm{a} / 100 \cdot 8.8+\mathrm{b} / 100 \cdot 183.5=\mathrm{m}
$$

Substitution of equation (1) into equation (2) results in the following formula:

$$
b=(m-8.8) / 1.747
$$


in which:

$b=$ percentage of hepatic tissue in the HTX-treated spleen

$\mathrm{m}=\mathrm{U} / \mathrm{g}$ GLDH measured in the homogenate of the HTX-treated spleen.

Mixed homogenates of spleen and liver tissue with known liver/spleen tissue ratio $(1,5,10,25,50$ and $75 \%$, respectively) were prepared. The amounts of GLDH per gram of mixed liver/spleen tissue, as found by actual measurements $(n=3)$ in these mixed homogenates corresponded to the calculated values, obtained with the formula. The data on GLDH content, measured in spleen and liver homogenates and in mixed liver/spleen homogenates, are compiled in Figure 4 . The conformity of calculated and actually measured GLDH content in liver/spleen mixtures allowed us to use the formula for determination of liver mass contained in HTX-treated spleens.

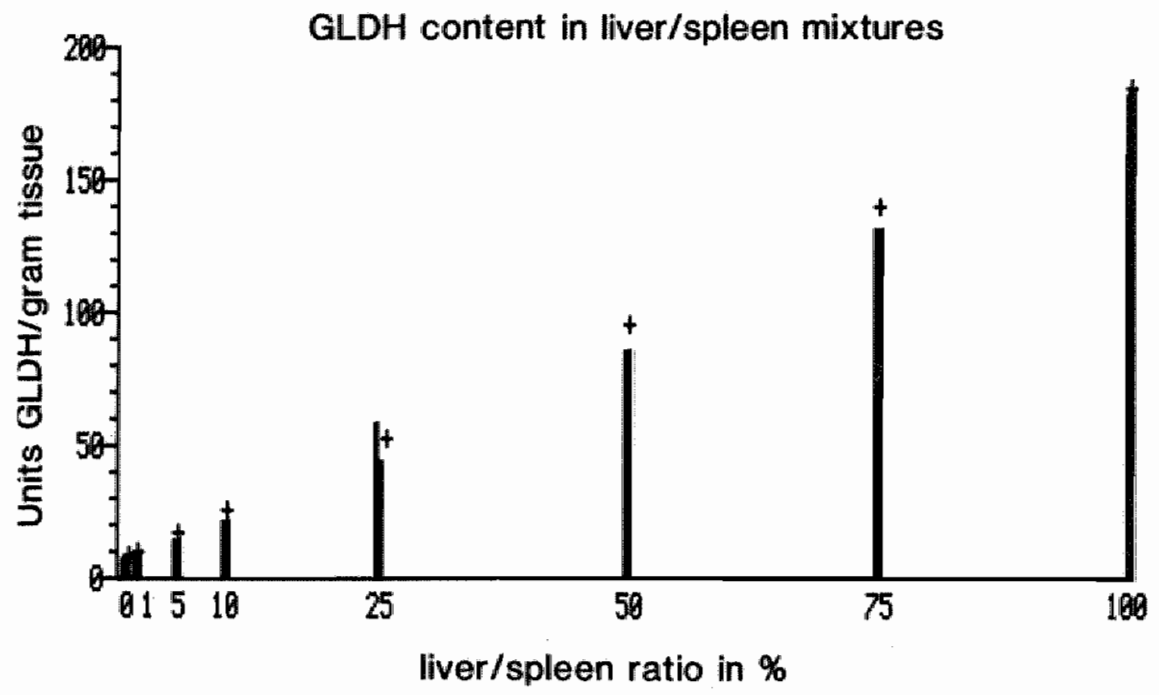

Figure 4. The actually measured GLDH contents in the liver/spleen mixtures were practically similar to the values obtained by calculation. Bars represent the measured GLDH content expressed in Units GLDH per gram of homogenized tissue (mean of 3 measurements); crosses $(+)$ represent the wallues obtained by calculation.

The entire spleen (with the exception of a very small biopsy) of the transplanted animals was used for preparation of the homogenate to be assayed on GLDH. The spleen biopsies demonstrated graft survival in all rats. By means of the formula, both the relative and absolute weight of intrasplenic liver tissue mass (in $\%$ and $\mathrm{mg}$, respectively) in all the individual rats were computed.

In Group I (animals that underwent HTX only) a rise in GLDH content of the spleen was observed between Group I-A and Group I-B that was statistically significant $(p<0.05)$. GLDH content increased from $22.9 \pm 1.4 \mathrm{U} / g$ (means $\pm S E M$ are 
given) at 12 weeks post-HTX (Group I-A) to $27.7 \pm 1.5 \mathrm{U} / \mathrm{g}$ at 20 weeks post-HTX (Group I-B). Spleen weights decreased slowly during the posttransplant period, from $435 \pm 13 \mathrm{mg}$ in Group I-A to $410 \pm 16 \mathrm{mg}$ in Group I-B ( $\mathrm{p}>0.05$ ). After calculation of the relative and absolute weights of intrasplenic liver mass in all transplanted rats, the mean values between groups were compared. The relative values at week 1.2 (Group I-A) and week 20 (Group I-B) post-HTX were $8.1 \pm 0.8 \%$ and $10.8 \pm 0.8 \%$, respectively. This difference was statistically significant $(\mathrm{p}<0.05)$. The correspondling absolute weights were $35.1 \pm 4.2 \mathrm{mg}$ and $44.4 \pm 3.7 \mathrm{mg}$ (this difference almost reached statistical significance, $0.1>p>0.05$ ).

In Group II, rats subjected to PH at 12 weeks post-HTX, the mean amount of GLDH contained in the spleen at 3 days after PH (Group II-A) amounted to $23.0 \pm 1.7 \mathrm{U} / \mathrm{g}$, whereas this value in Group II-B, at 8 weeks after PH (i.e. weeks 20 post-HTX), was $26.8 \pm 1.2 \mathrm{U} / \mathrm{g}$ (Group II-A vs Group II-B, $0.1>\mathrm{p}>0.05$ ). Mean spleen weight decreased from $446 \pm 7 \mathrm{mg}$ in Group II-A to $382 \pm 15 \mathrm{mg}$ in Group II-B $(\mathrm{p}<0.05)$. In the $\mathrm{PH}$-group, relative weights of liver tissue in the spleen amounted to $8.1 \pm 1.0 \%$ in Group II-A and to $10.3 \pm 0.7 \%$ in Group II-B $(0.1>p>0.05)$, absolute weights being $36.3 \pm 4.4 \mathrm{mg}$ and $39.4 \pm 1.9 \mathrm{mg}$, respectively. This increase was statistically not significant $(p>0.1)$. There were no statistically significant differences between Groups I-A and II-A, or between Groups I-B and II-B.

A summary of all relevant data pertaining to this part of the study is found in Table 3.

Table 3. Compiled data of enzymologic quantification of intrasplenic liver mass following HTX. Measurements at 12 and 20 weeks post-HTX ${ }^{a}$.

\begin{tabular}{|c|c|c|c|c|}
\hline & \multicolumn{2}{|c|}{12 weeks post-HTX } & \multicolumn{2}{|c|}{20 weeks post-HTX } \\
\hline & $\begin{array}{l}\text { Group I-A } \\
\text { (no PH) }\end{array}$ & $\begin{array}{l}\text { Group II-A } \\
\text { (PH) }\end{array}$ & $\begin{array}{l}\text { Group } 1-B \\
\text { (no PH) }\end{array}$ & $\begin{array}{l}\text { Group II-B } \\
\text { (PH) }\end{array}$ \\
\hline $\begin{array}{l}\text { GLDH content in the HTX- } \\
\text { treated spleen } \mathrm{U} / \mathrm{g}^{\mathrm{b}}\end{array}$ & $22.9 \pm 1.4$ & $23.0 \pm 1.7$ & $27.7 \pm 11.5$ & $26.8 \pm 1.2$ \\
\hline spleen weight in $\mathrm{mg}^{\mathrm{c}}$ & $435 \pm 13$ & $446 \pm 7$ & $410 \pm 16$ & $382 \pm 15$ \\
\hline $\begin{array}{l}\text { liver in spleen: } \\
\text { relative amount in \% }{ }^{0} \mathrm{~d}\end{array}$ & $8.1 \pm 0.8$ & $8.1 \pm 1.0$ & $10.8 \pm 0.8$ & $10.3 \pm 0.7$ \\
\hline absolute amount in mge & $35.1 \pm 4.2$ & $36.3 \pm 4.4$ & $44.4 \pm 3.7$ & $39.4 \pm 1.9$ \\
\hline
\end{tabular}

- means \pm SEM are given.

b significant differences were observed between Groups I-A and I-B, and between Groups II-A and II-B $(p<0.05)$.

c spleen weights in Group II-B were significantly lower $(p<0.05)$ than in the other groups.

d between Groups I-A and I-B, $\mathrm{p}<0.05$; between Groups II-A and II-B, $0.1>p>0.05$. Statistical differences were not present between Groups I-A and II-A, or between Groups I-B and II-B.

e between Groups I-A and I-B, $0.1>\mathrm{p}>0.05$. 
The potential of hepatocyte tranplantation (HTX) as an alternative treatment for enzyme deficiency disease or acute hepatic failure has received ample attention (1-11). Further research into HTX has been focused on the morphological features and metabolic functions of the transplanted liver cells (12-16). The most appropriate acceptor organ for the hepatocyte suspension appears to be the spleen (11-14). Intrasplenic HTX results in the formation of liver cell aggregates in the red pulp of the spleen. After nidation, the isolated hepatocytes regroupe and seem to reconfigurate, at least partially, the micro-architecture of the liver, forming hepatic cell cords with intercellular bile canaliculi $(4,5,11-14)$. Apart from maintaining their morphological integrity, the intrasplenic hepatocytes also retain long-term metabolic functions, as has been revealed by histochemical staining techniques $(4,5,12,13)$, scintigraphic studies (15-17), indocyanine green clearance and ammonia tolerance tests after a portacaval shunt procedure (13) and the persisting activity of bilirubin glucuronizing enzyme in congenitally jaundiced rats that were treated by HTX (5). Little is known yet about the cytokinetic behaviour of transplanted isolated hepatocytes. Precise knowledge of this aspect of HTX is desirable, since the efficacy of metabolic support of the hepatocellular transplant to the host is expected to be positively correlated with donor liver mass.

This study was designed to elucidate the cytokinetic behaviour of isolated hepatocytes grafted into the spleen. It provides definite proof of the spontaneous proliferative activity of these cells; the proportion of intrasplenic donor hepatocytes in Sphase, as detected by BrdU pulse labeling, was fairly constant in the animals studied (around $3 \%$ ), at 12 weeks, as well as at 20 weeks post-transplantation. Recently, it was demonstrated by autoradiography with ${ }^{3} \mathrm{H}$-thymidine that spontaneous proliferation of intrasplenic hepatocytes occurred in the first week posttransplantation, but at the end of the study (at 15 days after HTX) the ${ }^{3} \mathrm{H}$ thymidine labeling index of these cells had returned to $0 \%$ (22). Our data show that spontaneous proliferative activity of transplanted hepatocytes is present at 12 weeks post-HTX, and persists for at least 20 weeks after HTX. The short-living proliferative activity in the immediate post-transplantation period could possibly be explained by a DNA-synthesis initiating effect to the donor hepatocytes caused by the isolation procedure, as hepatic tissue is disrupted by proteolytic enzyme activity in the perfusion buffer. In this context it is worth mentioning, that pre-transplantation events can indeed influence the proliferative behaviour of donor hepatocytes, since hepatocytes, isolated from cirrhotic rats seem to proliferate more rapidly after HTX than normal hepatocytes (12). Our findings are in favour of continuous proliferative stimulation in the acceptor environment, rather than the expression of antecedent stimulatory events to the donor hepatocytes. This would be in support of the hypothesis, that a subpopulation of isolated hepatocytes survives the initial posttransplantation period and then starts to proliferate, which eventually results in the formation of real ectopic hepatic tissue (14). The hepatoproliferative stimulus 
present in the spleen is unknown. Both humoral and local factors can be involved. Whether these "hepatotrophic" substances are hormones (of which insulin and glucagon would be the most important) or a hepatocyte-specific growth-factor is subject of intensive research $(23,24)$. The fact, that intrasplenic hepatocytes show spontaneous mitotic activity while proliferative activity in the host liver is practically nonexistent, suggests that local factors play an important role in hepatoproliferative control of ectopic liver cells.

Partial hepatectomy $(\mathrm{PH})$ caused an explosive onset of proliferative activity in the remaining host liver. The sequence of events leading to restoration of weight of the liver following PH has been studied extensively (25-27). The rate of compensatory hyperplasia of the remaining liver lobes after PH in this study (see Table 2) was in agreement with earlier descriptions (25). The PI in the host liver increased from virtually $0 \%$ in the "'quiescent" pre-PH stage, to around $15 \%$ at the 2 nd day post-PH. Thereafter, the PI rapidly declined to about $5 \%$ on day 4 , and almost $0 \%$ (less than 1 per thousand) on day 7 post-PH. On day 10 however, a small, yet noticeable increase in PI up to $2 \%$ was observed again in the liver. The second phase proliferative response during the compensatory growth of rat liver after partial removal was reported before $(25,26)$. The intrasplenic donor hepatocytes also reacted to $\mathrm{PH}$ with a sudden rise in proliferation rate. Their initial proliferative response paralleled that of the host liver cells, although the increase of PI levels was less pronounced. The PI of intrasplenic hepatocytes increased from approximately $3 \%$, spontaneously present in intrasplenic hepatocytes of HTX-treated rats, to around $10 \%$ at the 2 nd day after PH. Thereafter, the PI dropped to the $5 \%$ level at day 4 post-PH. On day 7 , the PI was reduced to about $3 \%$, the level of spontaneous proliferative behaviour, and stayed around this level at day 10 and week 8 after PH. Obviously, proliferative activity of the intrasplenic hepatocytes can be influenced by a hepatoproliferative stimulus elsewhere in the host, indicating that (a) circulating humoral agent(s) exert(s) a modulating effect on the cytokinetic behaviour of the donor hepatocytes. Quantification of intrasplenic liver mass is of importance for the study of ectopic liver formation following intrasplenic HTX. In order to develop a method for the quantification of hepatic tissue present in the spleen, we analyzed the enzyme content of liver and spleen tissue to characterize a tissue specific enzyme spectrum. Organ specificity was obtained by determination of a single enzyme; glutamate dehydrogenase (GLDH). This enzyme of mitochondrial origin is only lost from cells following severe cellular injury (28). For the purpose of this study, GLDH appeared to be a useful marker enzyme for quantitative estimation of liver tissue. By means of GLDH assays in homogenates of the HTX-treated spleens, we were able to demonstrate that spontaneous mitotic activity in the donor hepatocytes did result in an increase of relative and absolute intrasplenic liver mass between the 12 th and 20th week post-HTX. The transient rise in proliferation rate in the hepatocellular transplant after $\mathrm{PH}$, did not result in an additional or accelerated increase of intrasplenic liver mass. At 3 days post- $\mathrm{PH}$, as well as at 8 weeks post-PH, intrasplenic liver mass was not higher in the PH Group II than in the non-PH Group I. It has 
been shown recently, that significant and rapid growth of preneoplastic and normal hepatocytes was obtained by simultaneous intrasplenic HTX and PH, when the proliferative response of the remaining host liver was inhibited by pretreatment with 2-acetylaminofluorene $(29,30)$.

In previous papers, morphometric analyses for determination of intrasplenic hepatocellular mass were used. These investigations were carried out on a single longitudinal H\&E stained section of the spleen $(13,29,30)$. In our hands, similar morphometric analyses led to inconstant results. We ascribed the inconstancy of our morphometric results to the inhomogeneous distribution of the liver cells contained in the spleen, and the sometimes ill-defined boundaries between splenic tissue and hepatocellular donor tissue. The results obtained with the present enzyme assay method were not affected by these factors, because the entire HTX-treated spleen was used for preparation of the homogenate to be assayed on GLDH.

In conclusion, this study provides unequivocal evidence of the spontaneous proliferative activity of isolated hepatocytes after nidation in the spleen. At 12 weeks and at 20 weeks posttransplantation, the proliferation index (PI) of intrasplenic liver cells amounted to approximately $3 \%$ as assessed by pulse labeling with $\mathrm{BrdU}$, a thymidine analogue. This slow, but continuous mitotic behaviour in the hepatocellular graft caused a significant increase of intrasplenic liver mass between the 12th and the 20 st week post-HTX. This parameter was determined by means of a newly developed method, based on GLDH content of grafted hepatic tissue. Partial removal of the host liver resulted in a modest, transient rise in PI of the donor hepatocytes contained in the spleen, but could not bring about an actual increase of intrasplenic liver tissue mass.

\section{Acknowledgements}

The authors owe thanks to Mrs. B. Engelen and Mrs. M. Reynders for technical assistance, and to Mrs. K. Spronck for preparation of the manuscript.

This paper has been accepted for publication in Transplantation.

Vroemen JPAM, Buurman WA, Maessen JG, Schutte B, Van der Linden CJ, Kootstra G.

The cytokinetic behaviour of donor hepatocytes after syngenic hepatocyte transplantation into the spleen.

Transplantation (in press).

\section{References}

1. Matas AJ, Sutherland DER, Steffes MW et al.

Hepatocellular transplantation for metabolic deficiencies: decrease of plasma bilirubin in Gunn rats. Science 1976; 192: 892 . 
2. Groth $C G$, Arborgh $B$, Björkén $C$, Sundberg $B$, Lundgren $G$.

Correction of hyperbilirubinaemia in the glucuronyl-transferasedeficient rat by intraportal hepatocyte transplantation.

Transplant Proc 1977; 9: 313.

3. Sutherland DER, Matas AJ, Steffes MW, Simmons RL, Najarian JS.

Transplantation of liver cells in an animal model of congenital enzyme deficiency disease: the Gunn rat.

Transplant Proc 1977; 9: 317.

4. Vroemen JPAM, Blanckaert N, Buurman WA, Heirwegh KPM, Kootstra G.

Treatment of enzyme deficiency by hepatocyte tranplantation in rats.

J Surg Res 1985; 39: 267.

5. Vroemen IPAM, Buurman WA, Heirwegh KPM, Van der Linden CJ, Kootstra $\mathrm{G}$.

Hepatocyte transplantation for enzyme deficiency disease in congenic rats.

Transplantation 1986; $42: 130$.

6. Demetriou AA, Whiting JF, Feldman $D$ et al.

Replacement of liver function in rats by transplantation of microcarrier-attached hepatocytes.

Science 1986; $233: 1190$.

7. Sutherland DER, Numata M, Matas AJ, Simmons RL, Najarian JS.

Hepatocellular transplantation in acute liver failure.

Surgery 1977; 82: 124.

8. Sommer BG, Sutherland DER, Matas AJ, Simmons RL, Najarian JS.

Hepatocellular transplantation for treatment of D-galactosamine-induced acute liver failure in rats. Transplant Proc 1979; 11: 578.

9. Makowka L, Falk RE, Rotstein LE et al.

Cellular transplantation in the treatment of experimental hepatic failure.

Science $1980 ; 210: 901$.

10. Makowka L, Rotstein LE, Falk RE et al.

Allogeneic and xenogeneic hepatocyte transplantation in experimental hepatic failure.

Tranplantation 1980; 30: 429 .

11. Minato M, Houssin D, Demma I et al.

Transplantation of hepatocytes for treatment of surgically induced thepatic failure in the rat.

Eur Surg Res 1984; 16: 162.

12. Mito $M$, Ebata $H$, Kusano $M$, Onishi $T$, Hiratsuka $M$, Saito $T$.

Studies on ectopic liver utilizing hepatocyte transplantation into the rat spleen.

Transplant Proc 1979; $11: 585$.

13. Mito $M$, Ebata $H$, Kusano $M$, Onishi $T$, Saito $T$, Sakamoto $S$.

Morphology and function of isolated hepatocytes transplanted into rat spleen.

Transplantation 1979; 28: 499.

14. Kusano M, Mito M.

Observations on the fine structure of longsurvived isolated hepatocytes inoculated into rat spleen.

Gastroenterology 1982; 82: 616.

15. Woods RJ, Fuller BJ, Attenburrow VD, Nutt LH, Hobbs KEF.

Functional assessment of hepatocytes after transplantation into rat ispleen.

Transplantation 1982; 33: 123 .

16. Cuervas-Mons $\mathrm{V}$, Cienfuegos $\mathrm{JA}$, Maganto $\mathbb{P}$ et al.

Long-term evaluation of isolated syngeneic hepatocytes transplanted into the normal rat spleen by

TC-99M-HIDA scintigraphy.

Transplantation 1985; 39: 87.

17. Vroemen JPAM, Van der Linden CJ, Buurman WA, Coenegracht J, Heirwegh KPM, Kootstra $G$. In vivo dynamic ${ }^{99 m}$ Tc-HIDA scintigraphy after hepatocyte transplantation - A new method for monitoring of graft function.

Eur Surg Res 1987; 19: 140.

18. Seglen PO.

Preparation of isolated rat liver cells.

Methods Cell Biol 1976; 13: 29.

19. Waynforth HB.

Partial hepatectomy. 
In: Experimental and surgical technique in the rat. Edited by: Waynforth HB. Academic press, London, UK, 1980, p. 137.

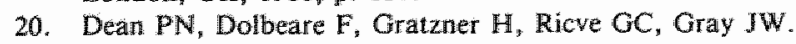

Cell cycle analysis using monoclonal antibody to BrdUdr.

Cell Tissue Kinetics 1984; 17: 427.

21. Schutte B, Reynders MMI, Bosman FT, Blijham GH.

Studies with anti-bromodeoxyuridine antibodies: II. Simultaneous immunocytochemical detection of antigen expression and DNA synthesis by in vivo labeling of mouse intestinal mucosa.

J Histochem Cytochem (in press).

22. Nordlinger $B$, Wang $S R$, Bouma $M E$ et al.

Can hepatocytes regenerate when transplanted in the spleen? (Abstract)

Eur Surg Res 1986; 18 (Suppl.. 1): 104.

23. Leffert HL, Koch KS, Moran T, Rubalcava B.

Hormonal control of rat liver regeneration.

Gastroenterology 1979; 76: 1470 .

24. Bucher NLR, Patel U; Cohen S.

Hormonal factors concerned with liver regeneration.

In: Hepatotrophic factors. Ciba Foundation Symposium 55. Edited by: Porter $\mathrm{R}$, Whelan J.

Elsevier/Excerpta Medica/North-Holland, Amsterdam 1978, p. 95.

25. Higgins GM, Anderson RM.

Experimentall pathology of the liwer. Restoration of the liver of the white rat following partial surgical removal.

Arch Path 1931; 12: 186.

26. Rabes HM.

Kinetics of hepatocellular proliferation as a function of the microvascular structure and functional state of the liver.

In: Hepatotrophic factors. Ciba Foundation Symposium 55. Edited by: Porter R, Whelan J.

Elsevier/Excerpta Medica/North-Holland, Amsterdam 1978, p. 31.

27. Kirsch RE, Terblanche $J$

Liver injury and regeneration.

In: The liver. Annual 2/1982. Edited by: Arias IM, Frenkel M, Wilson JHP. Excerpta Medica, Amsterdam 1982, p. 74.

28. Schmidt $E_{\text {n }}$ Schmidt FW.

Enzyme diagnosis in diseases of the liver and the biliary system.

In: Advances in clinical enzymology. Edited by: Schmidt E, Schmidt FW, Trautschold I, Friedel R. Karger, Basel, 1979, p. 239.

29. Finkelstein SD, Lee $G$, Medline A, Tatematsu M, Makowka $\mathbb{L}$, Farber E.

An experimental method for rapid growth of liver in spleen. The survival and proliferation of chemically induced preneoplastic hepatocytes in spleen.

Am J Pathal 1983; 110: 119.

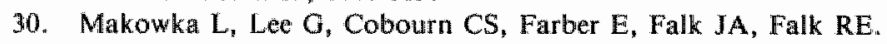

Allogeneic hepatocyte transplantation in the rat spleen under cyclosporine immunosuppression. Transplantation 1986; $42: 537$. 
In vivo dynamic ${ }^{99 m}$ Tc-HIDA scintigraphy after hepatocyte transplantation: a new method for the monitoring of graft function .

\section{Summary}

In vivo dynamic ${ }^{99 m} \mathrm{Tc}$-HIDA scintigraphy was tested as a method for graft function monitoring after hepatocyte transplantation (HTX). Bilirubin UDP-glucuronyl transferase-deficient rats received $10^{7}$ viable isolated hepatocytes from congenic nondeficient donors by intrasplenic injection. The transplanted rats were divided into two groups. Three months after HTX, one group underwent ${ }^{99} \mathrm{~m}$ Tc-HIDA scintigraphy, the other was subjected to bile analysis for the detection of bilirubin glucuronides (the presence of which would indicate biochemical activity of transplanted hepatocytes). Histological examination of the spleen of all animals was performed at the end of the experiment. The same experimental protocol was applied to sham-treated enzyme-deficient rats serving as controls.

The scintigraphic studies showed that spleens of transplanted rats did accumulate ${ }^{99 m}$ Tc-HIDA, this in contrast to sham-treated rats. Furthermore, time-activity curves of liver and spleen of HTX-treated rats showed similar kinetic patterns, suggesting a biologically normal function of the hepatocytes grafted in the spleen. Bilirubin glucuronides were excreted by transplanted rats, not by control rats. Histological examination of the spleen revealed hepatocyte survival in all HTX. treated rats. These findings demonstrated a correlation between increased splenic uptake of ${ }^{99 \mathrm{~m}} \mathrm{Tc}-\mathrm{HIDA}$ and biochemical activity and morphological survival of intrasplenic donor hepatocytes. In conclusion, in vivo dynamic ${ }^{95 \mathrm{~m}} \mathrm{Tc}-\mathrm{HIDA}$ scintigraphy appears to be an ideal method for noninvasive graft function monitoring affter intrasplenic HTX. 


\section{Introduction}

It has been firmly established that isolated rat liver cells, transplanted into the spleen of syngenic rats, survive and continue their metabolic activity (1-3). Further investigations have demonstrated that hepatocyte transplantation (HTX) may be considered as a new and promising therapeutic approach to enzyme deficiency disease (4-8). These studies were performed in a rat model of congenital jaundice caused by a total enzymatic defect of bilirubin conjugation in the liver. We reported that biochemical activity of grafted nondeficient donor hepatocytes could be demonstrated by determination of bilirubin glucuronides in bile of HTX-treated enzymedeficient animals $(7,8)$. A therapeutic effect was observed when cells from nondeficient allogenic donors were transplanted, but this effect was only transient, due to rejection of the donor cells (7). In contrast, treatment with hepatocytes from nondeficient congenic donors resulted in a long-lasting therapeutic effect (8). In this situation, the nondeficient donor hepatocytes survived and were shown to remain biologically active. Histological examination of the spleen and analysis of bile of the host were the cornerstones of the diagnosis of graft survival. Both methods have the disadvantage of requiring death of the animal and do not permit repeated measurements in individual rats during an extended period of time.

Static ${ }^{99 m}$ Tc-HIDA scintigraphy of the excised spleen was suggested by CuervasMons $(9,10)$ and Woods (11) as another approach to demonstrate functional graft survival. This method promised to be useful, but still possessed the same drawback as mentioned above. In search of a new, noninvasive and reliable technique to monitor graft survival after HTX, we studied in vivo dynamic ${ }^{99 \mathrm{~m}} \mathrm{Tc}$-HIDA scintigraphy on enzyme-deficient HTX-treated rats. Therefore, a correlation had to be demonstrated between biochemical activity of the donor hepatocytes (being the "'golden standard" for functional survival), their histological survival and splenic uptake of 99m Tc-HIDA. These parameters were studied in a new animal model of congenital bilirubin uridyldiphosphate(UDP)-glucuronyl transferase deficiency that was recently described (8). This model consists of two congenic rat strains: the enzymedeficient hyperbilirubinemic $\mathrm{R} / \mathrm{APfd}-\mathrm{j} / \mathrm{j}$ strain, and the nondeficient $\mathrm{R} / \mathrm{APfd}$ strain. The experimental set-up applied in this study offered not only the possibility of demonstrating the presence of metabolically active hepatocytes in the spleen, but it enabled us also to investigate the kinetics of ${ }^{99_{m}} \mathrm{Tc}$-HIDA uptake by donor hepatocytes.

This paper shows that in vivo dynamic ${ }^{99 m}$ Tc-HIDA uptake in the spleen correlates with both biochemical activity and histological graft survival of intrasplenic donor hepatocytes. In addition, it is demonstrated that splenic and hepatic ${ }^{99 \mathrm{~m}} \mathrm{Tc}-\mathrm{HIDA}$ uptake in the recipient follow an identical kinetic pattern. 


\section{Animals}

Inbred male Wistar R/APfd rats (275-350 g), obtained from the Proefdierencentrum, University of Leuven, Belgium, were used as hepatocyte donors. Conjugation of bilirubin in this rat strain is normal. Inbred male R/APfd-j/j rats (250-350 g) from the same source were used as recipients. The latter rat strain suffers from a congenital absolute deficiency of bilirubin UDP-glucuronyl transferase, and therefore exhibits a life-long hyperbilirubinemia of the unconjugated type $(j / j$ stands for homozygously jaundiced). Although deficient of the conjugating enzyme, this rat strain is perfectly capable of excreting conjugated bilirubin into bile (12). The $R / A P f d-j / j$ strain was obtained by repeated backcross-intercross mating ( 8 cycli 16 generations) between the GUNNXR/Pfd strain (being the donor of the gene coding for the deficient enzyme) and the normal Wistar R/APfd strain (13). Consequently, the R/APfd and R/APfd-j/j rats are congenic and histocompatible, and were shown to offer a unique model for the study of long-term graft function after hepatocyte transplantation (8). All animals were fed standard laboratory rat chow and water ad libitum.

\section{Hepatocyte transplantation}

Isolated hepatocytes were prepared by a modified two-step perfusion technique, originally described by Seglen (14). The portal vein of the anesthetized donor rat was cannulated and perfused with $250 \mathrm{ml} \mathrm{Ca}{ }^{++}$-ion-free Krebs-Henseleit solution at a flow rate of $20 \mathrm{ml} / \mathrm{min}$. After excision of the liver, perfusion was continued with Krebs-Henseleit solution containing $0.5 \mathrm{mg} / \mathrm{ml}$ collagenase (Sigma Chemicals, St. Louis, MO, USA) during approximately $12 \mathrm{~min}$. After digestion of the liver collagen matrix, the liver capsule was removed, and an initial cell suspension was obtained. This cell suspension was kept at $0-4^{\circ} \mathrm{C}$, filtered through a $100-\mu \mathrm{m}$ nylon sieve to eliminate cell clusters, and washed three times. Hepatocytes were suspended in Krebs-Henseleit solution containing $10 \mathrm{mg} / \mathrm{ml}$ fatty-acid-free bovine serum albumine (Sigma Chemicals) to a concentration of $10^{7}$ viable cells per $\mathrm{ml}$. Viability ranged from $85-95 \%$ as assessed by the trypan blue exclusion test. Prior to transplantation, the cell suspension was stored on melting ice.

The spleen of the host animal was exteriorized through a left subcostal incision under ether anesthesia. Hepatocyte transplantation was performed by slow injection of $1 \mathrm{ml}$ of cell suspension into the recipient spleen with a 25-gauge needle. Hemostasis was obtained by tamponade of the puncture hole with a cotton-tip. The splenic surface was marked by $3-\mathrm{mm}$ hemoclips in order to allow $\mathrm{X}$ ray localization in animals to be used for future scintigraphy. The spleen was fixed to the left abdominal wall with silk sutures to prevent anteroposterior overlap of the liver and spleen during scintigraphy. A left nephrectomy was performed simultaneously in order to 
rule out false positive results due to a high background count in the highly vascularized kidney.

Sham treatment consisted of the identical surgical procedure, but the recipients received an intrasplenic injection of $1 \mathrm{ml}$ of Krebs-Henseleit solution instead of 1 $\mathrm{ml}$ of hepatocyte suspension.

\section{9m Tc-HIDA scintigraphy}

The right jugular vein of each rat was catheterized with silicone tubing several hours before scintigraphy, in order to prowide reliable intravenous access without the risk of extravasation. Shortly before administration, ${ }^{99 m} \mathrm{TC}-\mathrm{HIDA} \quad{ }^{99 \mathrm{~m}} \mathrm{Tc}-\mathrm{N}$ (2,6-diethylacetanilido)-iminodiacetic acid; Solco Nuclear, Basel, Switzerland) was prepared in 1-ml aliquots, each containing $500 \mu \mathrm{Ci}$ of the hepatobiliary imaging agent. Immediately after induction of light ether anesthesia, the animal was placed in a supine position under a gamma camera (Philips MK3 LFOV, Eindhoven, The Netherlands), adapted with a pin hole collimator. Next, $500 \mu \mathrm{Ci}{ }^{99 \mathrm{~m} T \mathrm{~T}-\mathrm{HIDA} \text { was }}$ injected intravenously in $10 \mathrm{sec}$. During injection, dynamic scintigraphy was started and continued for a period of $10 \mathrm{~min}$, since the maximum in vivo hepatic uptake of ${ }^{99 \mathrm{~m}} \mathrm{Tc}$-HIDA in rats was known to be situated between 1 and $3 \mathrm{~min}$ after intravenous administration (15). Data were processed by means of a Philips processing and display system, and stored on a X/1216 cartridge disc drive for subsequent computation. In each rat, scintiphotographic images of the anatomical distribution of $99 \mathrm{~m}$ Tc-HIDA were displayed on a monitor in timeframes of $6 \mathrm{sec}$. Gamma activity during each timeframe (counts/timeframe) was calculated by computer over 4 fixed areas of $0.4 \mathrm{~cm}^{2}$, the so-called regions of interest (ROIs). Accordingly, over each ROI, 100 successive measurements were registered during the 10-min scanning period. The amount of activity measured per timeframe over each ROI was expressed as a percentage of the maximum activity measured at the center of the liver parenchyma, and was plotted on a time scale, resulting in a time-activity curve. Time-activity curves of the following ROIs were computed (Figure 1):

ROI 1: activity measured ower the heart;

ROI 2: activity measured over the margin of the left liver lobe;

ROI 3: activity measured over the spleen;

ROI 4: activity measured over the center of the liver parenchyma.

\section{Analytical methods}

Bile samples were examined for the presence of bilirubin mono- and diglucuronides by the liquid chromatographic assay as described by Muraca and Blanckaert (16). The smallest concentrations of bilirubin mono- and diglucuronides that are detectable and measurable by means of this assay are 10 and $20 \mathrm{nmol} / 1$, respectively. Total plasma bilirubin (TB) levels in all blood samples were measured by spectrophotometric absorbance (American Optical bilirubinometer type 10211, Buffalo, NY, USA). 


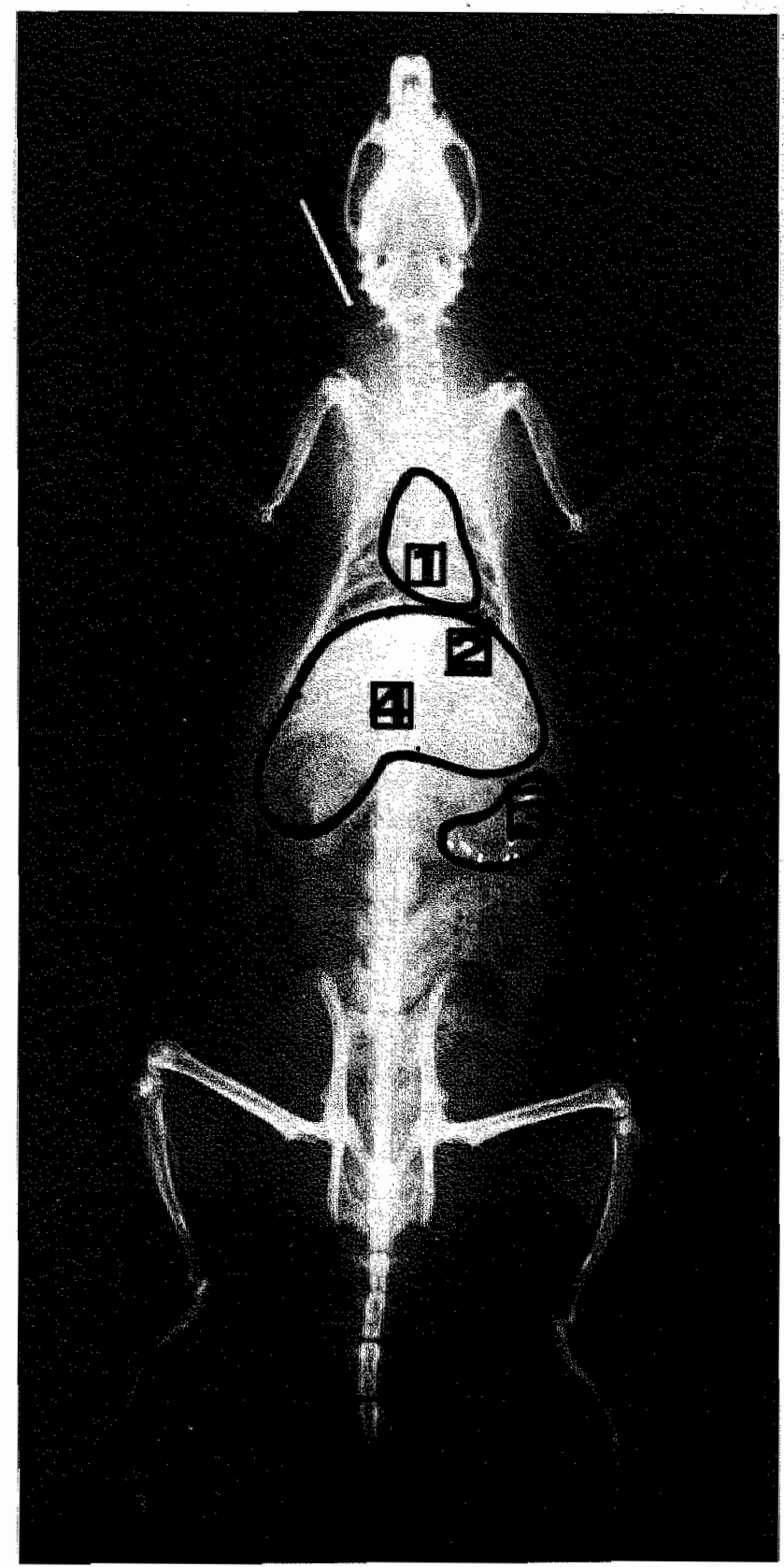

Figure 1. Soft-rissue $X$ ray of HTX-treated rat subjected to dynamic ${ }^{99 m}$ Tc-HIDA scintigraphy. Heart, liver and spleen are outlined, as well as the warious regions of interest (ROIs) studied during scintigraphy. The position of the hemoclipped spleen is clearly visible. ROI I: region of the heart; ROI 2: region of the left liver lobe; ROI 3: region of the splean; ROI 4: region of the center of the liver parenchyma. Approximately $\times 0.5$. 


\section{Histology}

Spleens of all animals were fixed in 10\% buffered formaldehyde and embedded in paraffin. After scintigraphy, at least two months were allowed for the disappearance of gamma activity before histologic processing was continued. Six- $\mu \mathrm{m}$ sections were stained with hematoxylin and eosin (H\&E), and by the periodic acid Schiff (PAS) procedure.

\section{Experimental design}

Ten $\mathrm{R} / \mathrm{APfd}-\mathrm{j} / \mathrm{j}$ rats underwent $\mathrm{HTX}$ with $10^{7}$ viable R/APfd hepatocytes and were divided into Groups I-A $(n=4)$ and I-B $(n=6)$. Animals of Group I-A were subjected to in vivo ${ }^{5 m}$ Tc-HIDA scintigraphy three months after transplantation. One rat of Group I-A was scanned twice with a time interval of 1 week. From the animals of Group I-B, blood samples were obtained before HTX and three months after HTX by paraorbital venipuncture for the determination of TB levels. After collection of the last bloodsample, the common bile duct of these animals was cannulated, and 3-hr bile specimens were sampled for analysis on bilirubin glucuronides. The animals of both groups were sacrificed after the diagnostic procedures, and their spleens were histologically examined. Ten $R / A P f d-j / j$ rats receiving sham treatment formed control Groups II-A $(n=4)$ and II-B $(n=6)$, which followed the same experimental protocol as Groups I-A and I-B, respectively. An outline of this experimental design is given in Table 1.

In each animal of Groups I-A and II-A, ${ }^{99 \mathrm{~m} T c-H I D A}$ uptake was recorded and scintigraphic results were compared. Between Groups I-B and II-B, pre- and postHTX TB levels and the biliary excretion of bilirubin glucuronides were compared.

Table 1. Outline of the experimental design.

\begin{tabular}{|c|c|c|c|}
\hline & $\begin{array}{l}99 \text { TC-HIDA } \\
\text { sointigraphy }\end{array}$ & $\begin{array}{l}\text { bile } \\
\text { analysis }\end{array}$ & $\begin{array}{l}\text { histological } \\
\text { examination }\end{array}$ \\
\hline $\begin{array}{l}\text { HTX-treated } \\
R / A P C-j / j \text { rats }\end{array}$ & $\begin{array}{l}\text { Group } \mathbb{I}-\mathrm{A} \\
(\mathrm{n}=4)\end{array}$ & $\begin{array}{l}\text { Group 1-B } \\
(n=6)\end{array}$ & $\begin{array}{l}\text { Group } 1-A \text { and } B \\
(n=10)\end{array}$ \\
\hline $\begin{array}{l}\text { sham treated } \\
R / A P f d-j / j \text { rats }\end{array}$ & $\begin{array}{l}\text { Group } \llbracket 1-A \\
(n=4)\end{array}$ & $\begin{array}{l}\text { Group II-B } \\
(n=6)\end{array}$ & $\begin{array}{l}\text { Group II-A and } B \\
(n=10)\end{array}$ \\
\hline
\end{tabular}

\section{Results}

\section{Scintigraphic studies after congenic hepatocyte transplantation}

Eight enzyme-deficient rats were subjected to $99 \mathrm{~m}$ Tc-HIDA scintigraphy. Four of these rats had been transplanted with hepatocytes from nondeficient donors (Group 


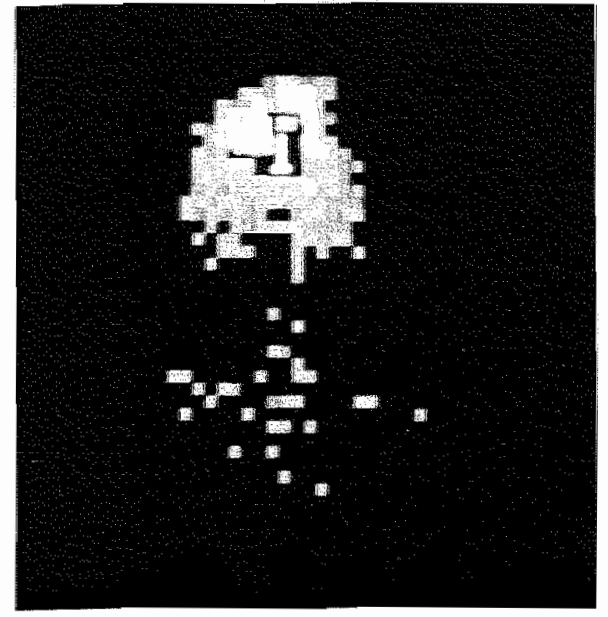

Figure 2. Typical scintiphotographic image at $12 \mathrm{sec}$ after intravenous administration of $500 \mu \mathrm{Ci}$ of ${ }^{99 \mathrm{n}} \mathrm{TC}$-HIDA. Accumulation of gamma activity is observed only over the heart (ROI I), iungs and region of the aorIa, and not (yet) over the other ROIs.

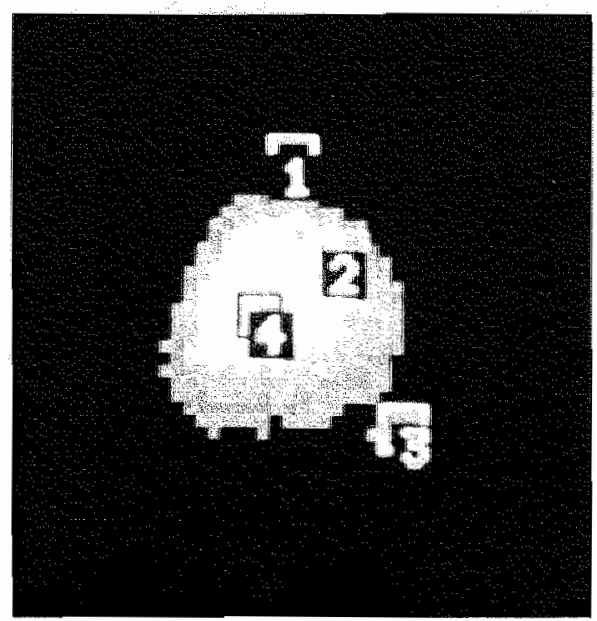

Figure 3. Scintiphosographic image of an HTX-treated rat at $120 \mathrm{sec}$ after ${ }^{2} \mathrm{Tc}$ HIDA administration. Phase of parenchymal uptake. Gamma activity over" the heart (ROI I) has disappeared, while accamulation of garnma activity over liver (ROIs 2 and 4) and spleen (ROI 3) is now observed.

I-A). Four sham-treated rats served as controls (Group II-A). In all 8 rats of both Groups I-A and II-A, a similar pattern of gamma activity over the heart (ROI 1), the margin of the left liver lobe (ROI 2) and the center of the liver parenchyma (ROI 4) was found.

Immediately after intravenous administration of $99 \mathrm{mc}$-HIDA (during the first 24 sec), high activity was measured over the heart (ROI 1), lungs and aorta, which decreased rapidly to background levels due to wash-out (Figure 2). At that time, elevated activity was not measured over the liver and spleen (ROIs 2, 4 and 3). Activity over the liver was increasing during approximately 1-3 min (phase of parenchymal uptake; Figure 3). Activity over the small bowel area rapidly increased after 3 min, as a sign of accumulation of $99 \mathrm{~m}$ Tc-HIDA in the gut after biliary excretion (not shown in the figures).

A difference in uptake of radioactivity between the two groups was observed over the spleen (ROI 3). In 3 out of 4 rats that underwent congenic HTX (Group I-A), a significant accumulation of gamma activity was measured over the spleen, indicating active splenic ${ }^{99 m}$ Tc-HIDA uptake. This resulted in photoscintigraphic images on which the spleen could be discerned as a separate entity from the liver (Figure 3). In sham-treated animals (Group II-A), the spleen was not recognized on photoscintigraphic images. As a result, the time-activity curves that were computed from each rat also revealed a typical difference between Groups I-A and II-A. In the 3 HTX-treated rats that showed splenic ${ }^{99 m}$ Tc-HIDA uptake, the time-activity 


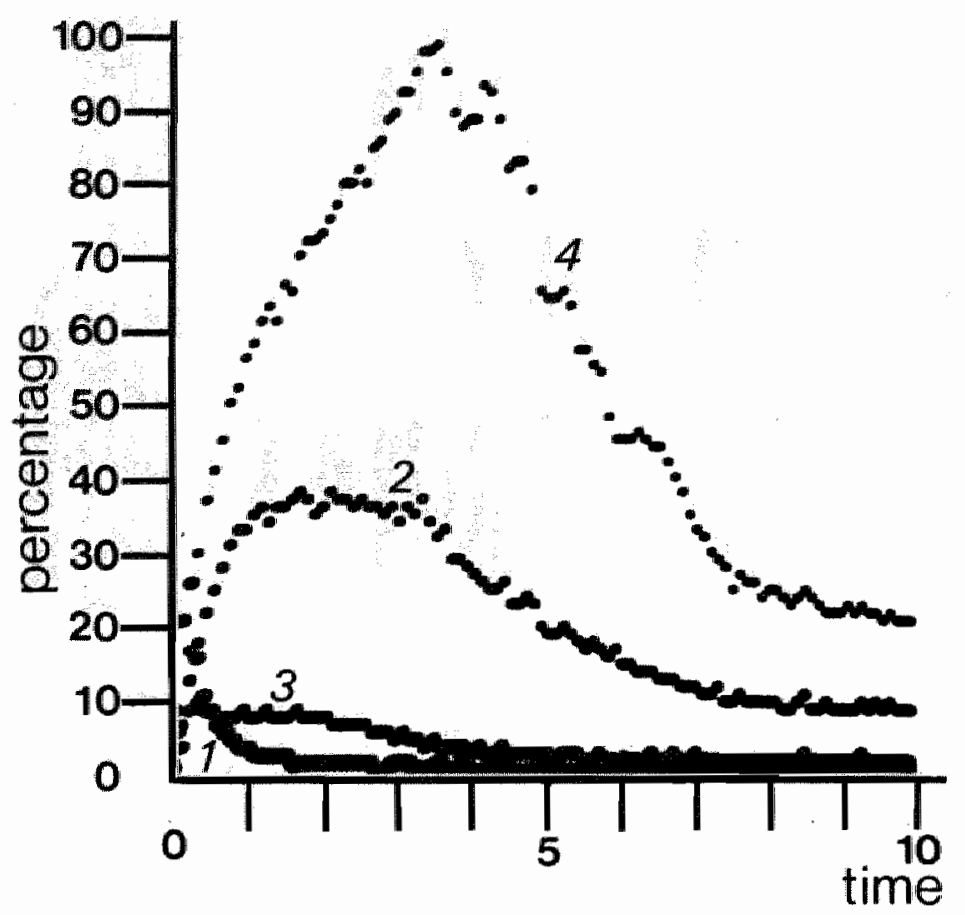

Figure 4. The time-activity curves of the 4 different ROIS studied in an HTX-treated rat (rat nr. 2, Group I-A; Table 2). Maximum activity is measured over the center of the liver parenchyma (ROI 4). The amount of gamma activity measured in other ROIs is expressed as a percentage of the maximum uptake in $\mathrm{ROI} 4$. Maximum activity registered over ROI 3 (spleen) amounted to $9.8 \%$ of the maximum activity measured over ROT 4 in this rat. Note the similar patterns of ${ }^{95 \%}$ TC-HIDA uptake in ROI 2,3 and 4. (time in minutes)

curves of the ROIs in liver and spleen (ROIs 2, 4 and 3, respectively) ran parallel to each other: an increased amount of gamma activity was observed during the parenchymal phase of uptake of the hepatobiliary imaging agent in both liver and spleen (Figure 4). The sham-treated rats (Group 11-A) did not show an increase of radioactivity over the spleen during scintigraphy. Time-activity curves of ROI 3 were flat in these animals and did not exceed the background levels that were observed in ROI 1 (heart) at more than $60 \mathrm{sec}$ after ${ }^{99 \mathrm{~m}}$ Tc-HIDA administration. The differences in splenic 99 mr Tc-HIDA uptake between the individual rats of the two groups are best illustrated by listing the maximum counts/timeframe that were measured over the 4 ROIs of each rat during the parenchymal phase of $99 \mathrm{mT}$ HIDA uptake. These values are shown in Table 2. For 3 rats in Group I-A, maximum counts/timeframe measured over ROI 3 (spleen) amounted to $8.9 \%, 9.8 \%$ and $9.3 \%$, respectively, of the maximum counts/timeframe measured over ROI 4 (center of the hepatic parenchyma). In Group II-A, the corresponding values virtually equalled the values found over the background region (ROI 1). These background values amounted to approximately $2 \%$ of the values found over the center of the 
Table 2. Maximum gamma activity measured over the 4 ROIs during the parenchymat phase of hepatic som Tc-HIDA uptake. ${ }^{\text {a }}$

Group I-A (HTX-treated rats)

\begin{tabular}{|c|c|c|c|c|}
\hline rat ner. & $\begin{array}{l}\text { ROI 1 } \\
\text { (heart) }\end{array}$ & $\begin{array}{l}\text { ROI } 2 \\
\text { (margin of the } \\
\text { left liver lobe) }\end{array}$ & $\begin{array}{l}\text { ROI } 3 \\
\text { (spleen) }\end{array}$ & $\begin{array}{l}\text { Rol } 4 \\
\text { (center of } \\
\text { liver) }\end{array}$ \\
\hline 1 & $(1.7 \%)$ & $(45 \%)$ & $(8.9 \%)$ & $(100 \%)$ \\
\hline 2 & $90 \quad(2.1 \%)$ & $1,624 \quad(38 \%)$ & $(9.8 \%)$ & $(100 \%)$ \\
\hline 3 & $82 \quad(1.8 \%)$ & $1,785 \quad(39 \%)$ & $425 \quad(9.3 \%)$ & $(100 \%)$ \\
\hline 4 & $97 \quad(1.6 \%)$ & $3,069 \quad(52 \%)$ & $123 \quad(2.1 \%)$ & $(100 \%)$ \\
\hline
\end{tabular}

Group II-A (sharn-treated rats)

\begin{tabular}{lllll} 
rat nr. & ROI 1 & ROI 2 & ROI 3 & ROI 4 \\
\hline 1 & $80(1.9 \%)$ & $1,694(41 \%)$ & $67(1.6 \%)$ & $4,132(100 \%)$ \\
2 & $76(1.6 \%)$ & $1,707(35 \%)$ & $119(2.4 \%)$ & $4,864(100 \%)$ \\
3 & $91(2.3 \%)$ & $1,834(46 \%)$ & $88(2.2 \%)$ & $3,972(100 \%)$ \\
4 & $86(1.9 \%)$ & $1,686(38 \%)$ & $61(1.4 \%)$ & $4,459(100 \%)$ \\
\hline
\end{tabular}

a Values are given in counts/timeframe, i.e. the number of counts of the timeframe during which maximum uptake was recorded in the studied ROIs.

Values between brackets represent the amount of gamma activity measured in each ROI expressed as a percentage of the maximum uptake in ROI 4. Consequently, the maximum uptake in ROI 4 is equivalent to $100 \%$.

liver (ROI 4) in both groups. Rat nr. 1 of the HTX-treated rats underwent a second scintigraphy one week later. Identical activity patterns over the studied ROIs were found. Rat nr. 4 of Group I-A did not show a measurable increase of gamma activity over the spleen. At autopsy, the spleen of this animal appeared to be dislodged and covered by the left liver lobe.

\section{Evaluation of the long-term biochemical function of donor hepatocytes}

Biochemical activity of grafted hepatocytes from nondeficient donors into bilirubin UDP-glucuronyl transferase-deficient recipients can elegantly be measured by quantification of bilirubin glucuronides in bile of the recipients $(7,8)$. In this study, absolute evidence for the presence of biochemically active donor hepatocytes in the HTX-treated rats was provided by the bile analyses. Excretion of bilirubin glucuronides was found in all bile specimens from enzyme-deficient rats that received nondeficient donor hepatocytes (Group I-B). In this group, $7.6 \pm 0.6 \%$ (mean \pm SEM) of biliary bilirubin was conjugated $(5.4 \pm 0.2 \%$ as bilirubin monoglucuronide plus $2.2 \pm 0.1 \%$ as bilirubin diglucuronide). The mean biliary concentration of bilirubin conjugates was $1.46 \mu \mathrm{mol} / 1$. In bile specimens of shamtreated rats (Group II-B), bilirubin conjugates were not found.

The formation of bilirubin glucuronides resulted in a reduction of TB levels in HTX-treated animals. Three months after HTX, TB levels in Group I-B (R/APfd- 
$\mathrm{j} / \mathrm{j}$ rats that were trealed with hepatocytes from $\mathrm{R} / \mathrm{APfd}$ donors) were reduced in comparison to pre-HTX levels (pre- versus post-HTX values: $149 \pm 6$ and $\mathbb{4} 42 \pm 8$ $\mu \mathrm{mol} / 1$, mean $\pm \mathrm{SEM}$, respectively), although this decrease did not reach statistical significance ( $p>0.05$, Wilcoxon rank test). In jaundiced rats that underwent a sham operation (Group II-B), TB levels remained constant (pre-versus post-HTX values: $152 \pm 6$ and $151 \pm 6 \mu \mathrm{mol} / \mathrm{l}$, respectively).

\section{Histological examination of the spleens}

Survival of hepatocytes in all spleens of the HTX-treated rats (Groups I-A and I-B) was observed. Clusters of hepatocytes were randomly scattered through the red pulp of the spleen, without reconfiguration of the typical hepatic architecture (Figure 5). Hepatocytes were of normal size, as seen in the liver. Glycogen depots were demonstrated by their PAS-positive staining properties. The binuclear appearance of some of these cells was also recognizable. This description is in accordance with the histological findings of other studies $(2,3)$.

Spleens of the sham-treated rats showed normal histological features.

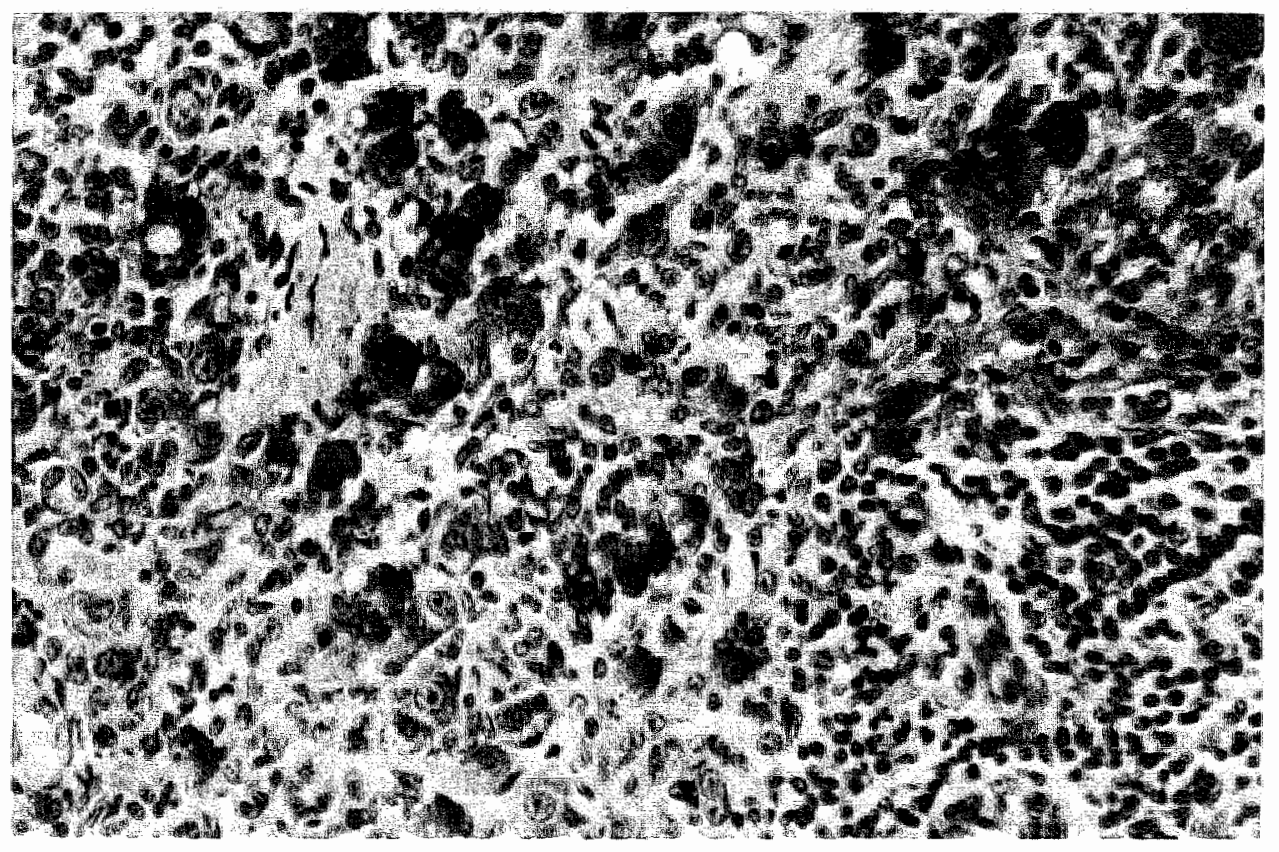

Figure 5. Histologically intact hepatocytes were found in the spleen of all HTX-treated rats. In this PAS stain, the cytoplasm appears bright purple-red (dark on this photograph) due to the PAS-positive staining properties of cytoplasmatic glycogen granules. Some hepatocytes show their typical binuclear appearance. However, reconfiguration of the characteristic atchitecture of the liver is not observed (PAS, $x$ 50). 


\section{Discussion}

The transplantation of isolated rat liver cells into the spleen of histocompatible recipient rats has been reported to result in the formation of functional ectopic liver tissue in the spleen (1-3). The potential therapeutic consequences of this relatively new transplantation technique have not gone unnoticed: HTX has been applied in the treatment of enzyme deficiency disease (4-8), acute liver failure (17-21) and druginduced cirrhosis (22) in several animal models with varying degrees of success. All investigators in this field used postmortem histological examination as the most important parameter for graft survival. Cuervas-Mons $(9,10)$ and Woods (11) were the first to use ${ }^{99 m} \mathrm{Tc}-\mathrm{HIDA}$ scintigraphy in the rat for the demonstration of the intrasplenic presence of functioning donor hepatocytes. Both authors administered ${ }_{99 \mathrm{~m}}$ Tc-HIDA to HTX-treated rats and measured gamma activity in the excised spleens shortly after sacrificing the animals. Histological survival of donor hepatocytes appeared to correlate with increased splenic uptake of this hepatobiliary imaging agent. ${ }^{99 m}$ Tc-HIDA is a radioisotope-labeled organic anion that is selectively taken up by the liver parenchymal cells, and secreted into the bile caniculi. Although there is no precise knowledge of the plasma proteins and/or enzymes involved, this transport takes place by a carrier-mediated organic anion pathway (23). Levi (24) demonstrated the existence of two cytoplasmic protein fractions, called the $\mathrm{Y}$ and the $Z$ protein, that play a major role in the hepatic uptake and excretion of organic anions. Further evidence of the necessity of intact cell metabolism required for ${ }^{99 m}$ Tc-HIDA uptake is provided by the fact that accumulation of ${ }^{99} \mathrm{~m}$ Tc-HIDA does not occur in necrotic liver tissue (15).

These data prompted us to perform in vivo dynamic ${ }^{99 m}$ Tc-HIDA scintigraphy in an animal model of enzyme deficiency, in which we previously had demonstrated long-term functional survival of donor hepatocytes grafted in the spleen (8). The purpose of the experiment was to evaluate this scintigraphic procedure as a reliable noninvasive method for monitoring graft survival. In 3 of the 4 HTX-treated rats of Group I-A, a significant accumulation of ${ }^{99} \mathrm{~m}$ Tc-HIDA uptake in the spleen was observed. This result was shown to be reproducible. In the fourth HTX-treated rat of Group I-A, overlap of the liver and spleen hampered scintigraphic visualization of the latter organ. Bilirubin glucuronides were demonstrated in the bile of all rats of the identically treated Group I-B. Morphologically intact hepatocytes were found in the spleens of all 10 rats of Groups $\mathbb{I - A}$ and I-B. In the corresponding control groups, there was, besides the obvious absence of intrasplenic hepatocytes, neither accumulation of gamma activity over the spleen, nor bilirubin glucuronide formation. Hence, it was possible to demonstrate a correlation between increased splenic uptake of ${ }^{99 \mathrm{~m}} \mathrm{Tc}-\mathrm{HIDA}$ and functional and histological survival of intrasplenic donor hepatocytes. This report offers a succinct description of the applied method, and also provides evidence for the validity of this technique in monitoring graft survival after HTX. 
It was of interest that time-activity curves over liver and spleen of HTX-treated rats ran parallel. This demonstrated that $99 \mathrm{~m}$ Tc-HIDA uptake by the intrasplenic hepatocytes followed the same kinetics as the uptake by the liver parenchyma, which may be considered as additional evidence for maintenance of physiological function of donor hepatocytes transplanted in the spleen.

Other foci of ${ }^{9915} \mathrm{Tc}-\mathrm{HIDA}$ accumulation than liver, spleen and gut (after biliary excretion at a later stage) were not encountered. Although expected, this negative finding was of importance, since the nidation of isolated hepatocytes in the lung has been described after HTX $(19,25)$. Apparently, overflow of single cells from the portal circulation through the caval route into the pulmonary vascular bed can occur. However, this phenomenon was not observed in our previous studies, in which we did not find signs of intrapulmonary nidated hepatocytes in lung biopsies after HTX.

The behaviour of hepatocytes after injection in the spleen has been subject of several studies $(1,3)$. Evidence exists that a majority of the donor cells degenerate and become necrotic, whereas a subpopulation survives and nidates in the reticuloendothelial matrix of the spleen (3). It is still a matter of controversy whether or not surviving hepatocytes start proliferating spontaneously after HTX. Some biochemical and histological observations appear to be in favour of this possibility $(2,3,8)$, but this could not be confirmed in other studies (9-11). Proliferation of hepatocytes in the host is not only of academic interest, since it may have important therapeutic consequences. It can reasonably be assumed that metabolic support provided by donor hepatocytes is to some degree proportional to the total of grafted hepatic mass. Therefore, beneficial effects of HTX might become apparent or more pronounced only after sufficient time has passed to allow for the growth of an adequate amount of donor liver tissue. In this respect, it is worth mentioning that druginduced proliferation seems possible (26). We hope that in vivo dynamic ${ }^{99 m} \mathrm{Tc}$ HIDA scintigraphy will be a valuable new tool for noninvasive graft function monitoring after HTX.

\section{Acknowledgements}

The authors are endebted to Mrs. K. Spronck for typing the manuscript, and to Dr. Th. Postmes for his valuable instructions in the laboratory. Ir. R. Leyten kindly provided the laboratory animals. Mr. H. Simons took care of the animals.

This paper has been published in European Surgical Research.

Vroemen JPAM, Van der Linden CJ, Bururman WA, Coenegracht J, Heirwegh KPM, Kootstra G.

In vivo dynamic ${ }^{99 m} \mathrm{Tc}-\mathrm{HIDA}$ scintigraphy after hepatocyte transplantation: a new method for monitoring of graft function.

European Surgical Research 1987; 19: 140. 


\section{References}

1. Kusano M, Mito $\mathrm{M}$.

Observations on the fine structure of long-survived isolated hepatocytes inoculated into rat spleen.

Gasiroenterology 1982; 82: 616.

2. Mito $M$, Ebata $H$, Kusano $M$, Onishi $T$, Hiratsuka $M$, Saito $\mathbb{T}$,

Studies on ectopic liver utilizing hepatocyte transplantation into the rat spleen.

Transplant Proc 1979; 11: 585.

3. Mito M, Ebata H, Kusano M, Onishi T, Saito T, Sakamoto S.

Morphology and function of isolated hepatocytes transplanted into rat spleen.

Transplantation 1979; $28: 499$.

4. Groth $\mathrm{CG}$, Arborgh B, Björkén C, Sundberg B, Lundgren G.

Correction of hyperbilirubinemia in the glucuronyltransferase-deficient rat by intraportal hepatocyte transplantation.

Transplant Proc 1977; 9: 313.

5. Matas AJ, Sutherland DER, Steffes $M$ WW at al.

Hepatocellular transplantation for metabolic deficiencies: Decrease of plasma bilirubin in Gunn rats.

Science 1976; 192: 892 .

6. Sutherland DER, Matas AJ, Steffes MW, Simmons RL, Najarian JS.

Transplantation of liver cells in an animal model of congenital enzyme deficiency disease: The Gunn rat.

Transplant Proc 1977: 9: 317.

7. Vroemen JPAM, Blanckaert N, Buurman WA, Heirwegh KPM, Kootstra G.

Treatment of enzyme deficiency by hepatocyte transplantation in rats.

J Surg Res 1985; 39: 267.

8. Vroemen JPAM, Buurman WA, Heirwegh KPM, Van der Linden CJ, Kootstra G.

Hepatocyte transplantation for enzyme deficiency disease in congenic rats.

Transplantation 1986; $42: 130$.

9. Cuervas-Mons $V$, Cienfuegos JA, Maganto $P$ et al.

Long-term evaluation of isolated syngeneic hepatocytes transplanted into the normal rat spleen by

Tc-99m-HIDA scintigraphy.

Transplantation $1985 ; 39: 87$.

10. Cuervas-Mons V, Maganto P, Cienfuegos JA et al.

Morphology and $99 \mathrm{~m}$ TC-HIDA functional study of isolated syngeneic hepatocytes transplanted into rat spleen.

Eur Surg Res 1981; 13 (Suppl): 60 .

11. Woods RJ, Fuller BJ, Attenburrow VD, Nutt LH, Hobbs KEF.

Functional assessment of hepatocytes after transplantation into rat spleen.

Transplantation 1982; 33: 123 .

12. Arias LM, Johnson $\mathrm{L}_{*}$ Wolfson $\mathrm{S}$.

Biliary excretion of injected conjugated and unconjugated bilirubin by normal and Gunn rats.

Am J" Physiol 1961; 200: 1091.

13. Leyten $R$, Vroemen JPAM, Blanckaert $N$, Heirwegh KPM.

The congenic normal $R / A P f d$ and jaundiced $R / A P f d-j / j$ rat strains. A new animal model of hereditary nonhaemolytic unconjugated hyperbilirubinaemia due to defective bilirubin conjugation.

Laboratory Animals 1986; 20: 130

14. Seglen $\mathrm{PO}$.

Preparation of isolated rat liver cells.

Meth Cell Biol 1976; 13: 29 .

15. Tjen HSLM.

Cholescintigraphy. The clinical application of $99 \mathrm{~m}$ Technetium-diethyl-IDA to the investigation of the liver and biliary tract.

Thesis at the University of Utrecht, The Netherlands (1979). 
16. Muraca $M$, Blanckent $\mathrm{N}$.

Liquid-chromatographic assay and identification of mono- and diester conjugates of bilirubin in normal serum.

Clin Chem 1983; $29: 1767$.

17. Cuervas-Mons $\mathbf{V}$, Cienfuegos JA, Maganto $P$ et al.

Time-related efficacy of liver cell isografts in fulminant hepatic fallure.

Transplantation 1984; 38: 23.

18. Makowka $L$, Rotstein LE, Falk RE et al.

Reversal of toxic and anoxic induced hepatic failure by syngeneic, allogeneic, and xenogeneic hepatocyte transplantation.

Surgery $1980 ; 88: 244$.

19. Minato M, Houssin D, Demma I et al.

Transplantation of hepatocytes for treatment of surgically induced acute hepatic failure in the rat.

Eur Surg Res 1984; 16: 162 .

20. Sutherland DER, Numata M, Matas AJ, Simmons RL, Najarian JS.

Hepatocellular transplantation in acute liver failure.

Surgery 1977; 82: 124.

21. Sommer BC, Sutherland DER, Simmons RL, Najarian JS.

Hepatocellular transplantation for experimental ischemic acute liver failure in dogs.

I Surg Res 1980; 29: 319.

22. Erolles $\mathrm{G}$, Maganto $\mathbf{P}$, Pinedo I et al.

Development of an experimental model of cirrhosis and its treatment by syngeneic hepatocyte

transplantation into the rat spleen.

Eur Surg Res 1983; 15 (Suppl): 27.

23. Harvey E, Loberg $M$, Ryan J, Sikorski S, Faith W, Cooper M.

Hepatic clearance mechanism of Tc-99m-HIDA and its effect on quantitation of hepatobiliary function. Concise communication.

J' Nucl Med 1979; 20: 310.

24. Levi AJ, Gatmaitan $Z$, Arias IM.

Two bepatic cytoplasma protein fractions, $Y$ and $Z$, and their possible role in the hepatic uptake of bilirubin, sulfobromophtalein and other anions.

J Clin Invest 1969; 48: 2156.

25. Selden C, Gupta S, Johnstone R, Hodgson $\mathbf{H J}$.

The pulmonary vascular bed as a site for implantation of isolated liver cells in inbred rats.

Transplantation 1984; 38: 81 .

26. Finkelstein $S_{\text {, Lee }} G$, Medline $A$, Tatematsu $M$, Makowka $L$, Farber $\mathbb{E}$.

An experimental method for rapid growth of liver in spleen. The survival and proliferation of chemically induced preneoplastic hepatocytes in spleen.

Am J Pathol 1983; 110: 119. 


\section{Summary and conclusions}

At present, a satisfying treatment for most hereditary enzyme deficiency diseases is not available. Experimental and clinical evidence exists that transplantation of the liver from a nondeficient donor may result in cure of hepatic based metabolic disorders. In clinical practice however, the indication for liver transplantation in such cases is the cirrhosis, due to or complicating the enzymatic defect, and not the deficiency itself. A more attractive approach to the treatment of these diseases would be the transplantation of liver cells. This technique would offer considerable advantages compared to whole (or auxilliary) liver transplantation, such as a less hazardous operative procedure, the possibility of long-term cryopreservation of isolated liver cells and the potential use of one donor organ for the benefit of multiple recipients. In this thesis aspects of experimental hepatocyte transplantation (HTX) relevant to the treatment of enzyme deficiency diseases were investigated.

An overview of the literature on HTX is given in Chapter 1. In addition, this chapter provides the rationale of and an introduction to the experimental work presented in this thesis. The first reports on HTX describe a therapeutic effect of HTX in the Gunn rat. This rat strain suffers from a total lack of bilirubin UDP-glucuronyl transferase (BGT), and is congenitally jaundiced. After HTX with hepatocytes from nondeficient donor rats, total plasma bilirubin (TB) levels decreased in the recipient Gunn rats. The mechanism of action of HTX, underlying this therapeutic effect, was subject of investigation in the first experiments, which are described in Chapter 2. A significant, but only transient decrease in total plasma bilirubin (TB) levels was obtained, when allogenic hepatocytes from normal Wistar rats were transplanted into the spleen of the Gunn rat. In Gunn rats treated with Gunn hepatocytes TB did not decline. Rejection of the Wistar donor celis occurred, whereas the syngenic Gunn hepatocytes survived in the spleen. Analysis of bile of immunosuppressed Gunn rats that received Wistar hepatocytes, demonstrated the presence of the products of the missing enzyme BGT, bilirubin mono- and diglucuronide. Bile from immunosuppressed Gunn rats that had received non-viable hepatocytes, Gunn hepatocytes or suspension buffer did not contain bilirubin glucuronides. Sequential bile analyses in non-immunosuppressed HTX-treated Gunn rats showed that the appearance of BGT activity was only transient because of rejection of the Wistar liver cells. It was concluded that the decrease of TB in the Gunn rat after HTX was caused by the appearance of BGT activity, provided by metabolically active non- 
deficient donor hepatocytes. Rejection of these donor hepatocytes abrogated the therapeutic effect.

In order to avoid rejection of normal donor hepatocytes in this animal model of congenital jaundice, the enzymatic defect of the Gunn rat was "transferred" to a normal inbred Wistar rat. This was achieved at the "Proefdierencentrum" (Laboratory Animal Centre) of the Catholic University of Leuven, Belgium. After eight backcross-intercross breeding cycles, starting with the inbred Gunn rat strain (as donor of the "jaundice gene") and the inbred normal R/APfd Wistar strain, the new $R / A P f d-j / j$ rat strain ( $\mathrm{j} / \mathrm{j}$ stands for homozygously jaundiced) was obtained. Blood and bile analyses provided evidence that this rat strain exhibited the identical enzymatic defect as the Gunn rat. In a series of transplantation experiments (including HTX) and in vitro monitoring of histocompatibility, it was demonstrated that the new R/APfd-j/j rat was congenic and fully histocompatible with the R/APfd Wistar rat. The development and characterization of biochemical and biological features of this rat strain are described in Chapter 3.

In Chapter 4 , the R/APfd-j/j strain was used to study whether intrasplenic HTX resulted in a long-term therapeutic effect. A permanent fall in TB was observed in these BGT-deficient rats after treatment with R/APfd donor cells. At six months post-HTX, BGT activity was present in the recipient $R / A P f d-j / j$ rats as assessed by the presence of bilirubin glucuronides in their bile collections. Hepatocyte survival was demonstrated in all recipients by histological examination of the spleen. Splenectomy performed at 11 weeks post-HTX reduced the biliary excretion of bilirubin glucuronides, but did not abolish it. This showed that part of the donor cells nidated and survived in the liver and also remained functional in this organ.

The spleen is generally accepted as a suitable location for inoculation of isolated hepatocytes, because in this organ both survival and histological examination of the hepatocellular graft are possible. In view of the fact that the pancreas is of the same embryological origin as the liver and produces hormones to which "hepatotrophic" properties are ascribed (insulin and glucagon), the pancreas was considered as a possible acceptor organ for hepatocytes. Morphology and function of hepatocytes transplanted into the pancreas of syngenic or congenic recipient rats were studied in Chapter 5. Hepatocytes formed aggregates in the interlobular spaces and survived with intact morphology. Histological, cytochemical and functional studies undertaken in the $\mathbb{R} / \mathrm{APfd}-\mathrm{j} / \mathrm{j}$ rat at 3 months post-HTX provided definite proof for the intact metabolic activities of intrapancreatic R/APfd liver cells. However, intrasplenic HTX appeared to be more effective than intrapancreatic HTX in the treatment of the R/APfd-j/j rat, since biliary excretion of bilirubin glucuronides and the lowering of TB were more pronounced in the recipients with an intrasplenic transplant.

In the preceding chapters, the therapeutic effect of HTX was clearly demonstrated. In spite of this, normalization of TB levels was never attained. It could reasonably be assumed, that the number of grafted donor hepatocytes was the limiting factor determining the therapeutic result in this enzyme deficiency disease. A slow but 
progressive decline of TB in the six months posttransplant course of HTX-treated $\mathrm{R} / \mathrm{APfd}-\mathrm{j} / \mathrm{j}$ rats (Chapter 4 ) suggested that proliferation of the R/APfd hepatocytes resulted in an increase of donor liver mass. On the other hand, signs of mitosis were not observed on histological examination. Data in the literature concerning the proliferative behaviour of transplanted hepatocytes were often contradictory. The cytokinetic behaviour of intrasplenic donor hepatocytes was studied in Chapter 6 . Immunocytochemical visualization of S-phase cells, pulse labeled with a thymidine analogue (bromodeoxyuridine, BrdU), revealed that syngenic donor hepatocytes proliferated at a fairly constant rate (their proliferation index amounted to about $3 \%$ ). These studies were performed at 12 weeks and at 20 weeks post-HTX. Furthermore, a method, based on hepatocellular GLDH content, was developed for the quantification of liver tissue present in spleen. Evidence was provided that continuous proliferation in the hepatocellular transplant did result in an increase of total intrasplenic liver mass. After partial resection of the host liver, the intrasplenic hepatocytes exhibited a shortliving increase in proliferation index. However, this did not result in increased intrasplenic liver mass. It was postulated that the proliferative stimulus provided by partial hepatectomy was of too short duration to have a demonstrable effect on intrasplenic liver mass.

In previous reports, graft survival was verified by postmortem histological examinations of the spleen of the HTX-treated rats. In Chapter 7, in vivo dynamic ${ }^{99 \mathrm{~m}} \mathrm{Tc}$ HIDA scintigraphy was evaluated for the monitoring of hepatocellular graft survival. ${ }^{99 m}$ Tc-HIDA is a technetium-labeled hepatobiliary imaging agent that is rapidly excreted into bile. Scintigraphic studies were performed in R/APfd-j/j rats that had undergone intrasplenic HTX with $\mathrm{R} / \mathrm{APfd}$ hepatocytes 3 months before. These studies showed that the spleens of these rats accumulated ${ }^{99 \mathrm{~m}} \mathrm{Tc}$-HIDA. Histological examination of the spleen and bile assays on the presence of bilirubin glucuronides confirmed functional survival of the R/APfd hepatocytes. The kinetics of ${ }^{99 m} \mathrm{Tc}$-HIDA uptake in the HTX-treated spleens were similar to that of ${ }^{99 \mathrm{~m}} \mathrm{Tc}$ HIDA uptake in the liver parenchyma, indicating normal function of the intrasplenic hepatocytes. It was concluded that ${ }^{99 \mathrm{~m}} \mathrm{Tc}-\mathrm{HIDA}$ scintigraphy can be considered as a successful method for graft monitoring after intrasplenic HTX. This method would allow repeated investigations in the individual animal and might be a valuable tool in the monitoring of intrasplenic liver mass.

In summary, the investigations presented in this thesis have demonstrated that isolated hepatocytes survive and continue their metabolic activity after transplantation into an appropriate acceptor organ, when rejection is avoided. The sustained function of donor liver cells can be applied in the treatment of hepatic based inborn errors of metabolism, as was shown in a rat model of congenital enzyme deficiency disease.

As to the potential clinical applications of HTX, many questions still go unanswered. However, the possibilities for future use of this transplantation method now seem to crystalize. Initially, HTX was proposed as a new treatment modality for 
enzyme deficiency disease and life-threatening liver failure. Work of others has provided evidence that transplantation of intact hepatocytes is not required for a therapeutic effect in acute liver failure. A still undefined heat-stable factor released from liver cells, which was also present in liver cytosol, appeared to enhance liver regeneration and was found to be responsible for the improved cure rate in acute hepatic failure. In contrast, in this thesis it has been shown, that long-term success of HTX in the treatment of enzyme deficiency disease is dependent on the survival of nondeficient donor hepatocytes. In view of these facts, the potential clinical application of HTX at present may be the treatment of enzyme deficiency diseases. The applicability of HTX for treatment of enzyme deficiency diseases in clinical practice may be restricted by the quantity of transplanted liver cells. This quantity might be too small to achieve complete cure of the deficiency (as was observed in the animal model used in the presented studies). It should be noted however, that in the specific enzymatic defect of the BGT-deficient rat, the amount of donor liver tissue required for the conjugation of all bilirubin may be higher than the amount that would be needed in the treatment of other enzyme deficiencies. In many enzyme deficiency states, the presence of a minute quantity of the lacking enzyme would suffice to eliminate symptomatic disease (e.g. phenylketonuria). In this context, it is also worth mentioning that spontaneous proliferation of donor hepatocytes transplanted into the spleen resulted in a steady increase of donor liwer tissue mass. This event might well have clinical consequences. The possibility to influence the proliferative activity of the hepatocellular transplant is relevant in this respect.

The combination of HTX and DNA technology would be an important and intriguing dlevelopment. In principle, it is now feasible to transfect isolated or cultured hepatocytes (obtained from an enzyme-deficient patient) with the DNA sequence coding for the lacking enzyme. Autotransplantation of these transfected cells would obviate the need of immunosuppression and could result in cure of the patient. The data obtained in the studies of this thesis provide a rational basis for such treatment. Although further research into HTX is required, the conclusion seems justified that HTX offers perspectives for future clinical use in the treatment of enzyme deficiency diseases. 


\section{Résumé et conclusions}

Actuellement il n'existe pas encore de thérapeutiques satisfaisantes pour la plupart des erreurs innées du métabolisme. Des études expérimentales et cliniques ont montré que la greffe d'un foie prélevé sur un donneur sain permet la guérison des déficiences enzymatiques d'origine hépatique. La seule indication de transplantation hépatique dans ces cas réside davantage dans la cirrhose consécutive ou complicant le déficit enzymatique, plutôt que dans la déficience enzymatique elle-même. La transplantation d'hépatocytes isolés constitue une alternative attractive du traitement de ces maladies. Cette technique offre de nombreux avantages par rapport à la greffe de l'organe entier. L'intervention chirurgicale comporte moins de risques, les cellules du donneur peuvent être conservées par cryopréservation, et plusieurs patients peuvent être traités à partir des hépatocytes issus d'un seul organe donneur. Dans cette thèse certains aspects de la transplantation hépatocellulaire expérimentale sont étudiés. Ils se rapportent au traitement des déficiences enzymatiques héréditaires.

Le Chapitre 1 présente une revue de la littérature sur les résultats obtenus jusqu'à ce jour avec la greffe expérimentale d'hépatocytes. Ce chapitre contient en outre une introduction des expériences accomplies. Les premières tentatives de transplantation hépatocellulaire décrivent comment la greffe d'hépatocytes permet une réduction de l'hyperbilirubinémie causée par une déficience héréditaire totale de l'enzyme bilirubine glucuronyltransférase dont souffrent les rats Gunn. Cet enzyme lie le pigment biliaire (bilirubine) à un glucide, permettant son excrétion aisée dans la bile. L'absence totale de cet enzyme cause chez le rat étudié un ictère permanent. Il est apparu que la bilirubinémie du rat Gunn diminuait après transplantation d'hépatocytes de rats normaux. Dans le Chapitre 2 on a étudié comment la greffe d'hépatocytes pouvait induire cet effet thérapeutique. Une réduction manifeste, bien que temporaire, de la bilirubinémie a été obtenue après injection dans la rate des rats Gunn d'hépatocytes de rats sains (greffe allogénéique). Lorsque des hépatocytes de rats Gunn furent utilisés (greffe syngénéique) la bilirubinémie initiale ne s'est pas modifiée. Les expérimentations ont montré que les hépatocytes sains étaient rejetés, tandis que les hẻpatocytes Gunn étaient tolérés par le receveur Gunn. L'analyse de la bile des rats Gunn temporairement immunodéprimés (par irradiation) a montré que, en la présence d'hépatocytes normaux fonctionnels dans le rat Gunn, les produits de l'activité enzymatique déficiente (les glucuronides de bilirubine) étaient formés. Ces substances ne furent pas retrouvées lorsque des hépatocytes normaux détruits artificiellement ou des hépatocytes Gunn étaient greffés. En l'absence de tout traitement immunosuppresseur, on nota que la bile des rats Gunn, greffés à l'aide des hépatocytes normaux, ne contenait des glucuronides de bilirubine que pendant un temps court. A partir de ces données on a conclu que la chute de bilirubinémie chez le rat Gunn, après une greffe d'hépatocytes sains, était causée par l'apparition de l'enzyme ab- 
sent contenu dans les hépatocytes greffés. L'effet thérapeutique disparaissait en même temps que le rejet des cellules greffées.

Afin d'étudier les résultats de la transplantation hépatocellulaire à long terme chez le rat ictérique, il était nécessaire que les hépatocytes des rats normaux ne soient pas rejetés. Dans le centre d'animaux de laboratoire (Proefdierencentrum) de la Katholieke Universiteit Leuven, Belgique, une nouvelle lignée de rats a été créée, à partir du rat Gunn et d'une lignée de rats Wistar normaux (la lignée R/APfd). Cette nouvelle lignée $(\mathrm{R} / \mathrm{APfd}-\mathrm{j} / \mathrm{j})$ avait la même déficience enzymatique que le rat Gunn $(\mathrm{j} / \mathrm{j}$ indique la déficience enzymatique héréditaire), mais possédait pour le reste les caractéristiques tissulaires de la lignée normale R/APfd. Dans ce modèle, les cellules du rat R/APfd ne sont plus reconnues comme "étrangères" par le rat $R / A P f d-j / j$ et donc plus rejetées (lignées congénéiques). La création de cette nouvelle lignée de rats $\mathrm{R} / \mathrm{APfd}-\mathrm{j} / \mathrm{j}$ est décrite dans le Chapitre 3, ainsi que les expériences qui ont été faites pour démontrer aussi bien la déficience enzymatique que la congénéicité avec la lignée R/APfd.

Dans le Chapitre 4, on a utilisé cette nouvelle lignée de rats pour vérifier si la greffe d'hépatocytes avait un effet thérapeutique durable sur le rat présentant une déficience enzymatique. On nota une réduction permanente de la bilirubinémie chez le rat $\mathrm{R} / \mathrm{APfd}-\mathrm{j} / \mathrm{j}$, après injection intrasplénique d'hépatocytes $\mathrm{R} / \mathrm{APfd}$. Six mois après la transplantation, la bile des animaux greffés contenait toujours des glucuronides de bilirubine, signe de la présence de l'enzyme déficient. Il fut également démontré avec des techniques histologiques que les hépatocytes du donneur sain survivaient dans la rate des rats transplantés. Après splénectomie, il apparaissait que des glucuronides de bilirubine étaient toujours produits chez le rat receveur en moindre quantité toutefois. Cette observation a été attribuée à la migration intrahépatique par voie portale des cellules du donneur pendant l'injection intrasplénique.

En général la rate est considérée comme un endroit approprié pour la greffe d'hépatocytes, non seulement parce que ces cellules peuvent y survivre, mais aussi parce qu'elles peuvent y être étudiées facilement à l'aide de techniques histologiques courantes. Etant donné l'origine embryologique commune du foie et du pancréas et le fait que ce dernier soit la source d'hormones hépatotrophiques (insuline et glucagon), des expériences d'implantation intrapancréatique d'hépatocytes ont été réalisées et rapportées dans le Chapitre 5. On a pu observer que les hépatocytes en provenance de rats syngénéiques ou congénéiques survivaient dans les espaces interlobulaires du pancréas et montraient toujours des signes fonctionnels 3 mois après la transplantation. Après injection intrapancréatique d'hépatocytes R/APfd dans les rats $R / A P f d-j / j$, on nota une diminution de la bilirubinémie et la formation de glucuronides de bilirubine. Cependant, dans ces mêmes conditions expérimentales, il apparaissait que la transplantation d'hépatocytes dans la rate était plus efficace que dans le pancréas, compte tenu de la quantité des glucuronides de bilirubine formés. Les expériences précédentes ont clairement démontré que la greffe d'hépatocytes avait un effet thérapeutique sur le rat ictérique. Malgré cela, une normalisation de la biliburinémie ne fut cependant pas observée. La cause réside probablement dans un nombre trop restraint des cellules greffées. Dans les expériences décrites au Cha- 
pitre 4, on a noté, après la transplantation d'hépatocytes R/APfd dans la rate des rats ictériques $R / A P f d-j / j$, dans un premier temps une chute rapide de la bilirubinémie, suivie par une diminution plus lente. Ceci laisserait supposer qu'il existe une réplication des hépatocytes $\mathrm{R} / \mathrm{APfd}$, bien qu'on n'ait jamais mis en évidence de signes histologiques de division cellulaire au cours de nos expériences. Les données de littérature étaient également souvent contradictoires en ce qui concerne la prolifération des hépatocytes greffés. Cette question a été étudiée dans le détail dans le Chapitre 6. On se servit alors d'une technique immunocytochimique grâce à laquelle les cellules en phase " $S$ " du cycle cellulaire sont directement visibles dans les préparations histologiques.

Ainsi il a été possible de prouver que les hépatocytes syngénéiques, greffés dans la rate, étaient le siège de division cellulaire. Les études ont été réalisées 12 et 20 semaines après la transplantation. L'indice de prolifération (c'est à dire la proportion des hépatocytes qui est en phase " $S$ " à un moment donné) était d'environ $3 \%$. En outre, nous avons développé une méthode enzymologique permettant de mesurer la quantité de tissu hépatique dans la rate. La concentration de l'enzyme GLDH (existant dans le foie en quantité importante et dans la rate en quantité beaucoup plus faible) a été mesurée dans le tissu splénique des rats greffés. A partir de ces données la quantité de tissu hépatique intrasplénique a pu être déterminée. A l'aide de cette mếthode on a pu ainsi démontrer que la prolifération continue des hépatocytes du donneur entrainait une augmentation de la masse de tissu hépatique dans la rate. Après résection partielle du foie du receveur, une régénération hépatique rapide était observée. Dans ces conditions la division des hépatocytes intraspléniques était également stimulée. Cependant, on ne nota pas une augmentation supplémentaire du tissu hépatique du donneur par rapport à sa croissance spontanée. La durée de la stimulation proliférative, induite par l'hépatectomie partielle, était probablement trop courte pour entrainer un effet mesurable.

Au cours des expériences précédentes, on a pu vérifier la survie des hépatocytes greffés à l'aide d'un examen histologique de la rate. Pour cela il fallait sacrifier l'animal receveur. Dans le Chapitre 7 on évalue une nouvelle méthode d'étude de survie de la greffe: la scintigraphie dynamique ${ }^{99 m}$ Tc-HIDA. ${ }^{99 m}$ Tc-HIDA est une substance radioactive absorbée spécifiquement par les hépatocytes et ensuite éliminée par voie biliaire. ${ }^{99 m} \mathrm{Tc}-H I D A$ était absorbée par la rate des rats $\mathrm{R} / \mathrm{APfd}-\mathrm{j} / \mathrm{j}$ qui avaient reçu une greffe intrasplénique d'hépatocytes R/APfd 3 mois auparavant. Cela n'était pas observé au niveau du tissu splénique des rats $R / A P f d-j / j$ non transplantés. Par la corrélation des données de l'histologie, de la scintigraphie et de la détermination biologique des glucuronides de bilirubine dans la bile, on a démontré l'existence d'une association entre l'absorption de ${ }^{99 \mathrm{~m}}$ Tc-HIDA par la rate et la présence d'hépatocytes fonctionnels intraspléniques. Il ressortait en outre que la vitesse $d^{x}$ absorption et d'élimination de ${ }^{99 \mathrm{~m}} \mathrm{Tc}$-HIDA par la rate était semblable à celle du foie. Ceci illustrait un métabolisme normal des hépatocytes greffés. En principe, la scintigraphie ${ }^{99 \mathrm{~m}} \mathrm{Tc}$-HIDA permet des mesures itératives sur un même animal, et est donc une méthode élégante pour examiner la survie de la greffe hépatocellulaire. 
En résumé, les expériences décrites dans cette thèse ont démontré que des hépatocytes isolés et transplantés dans un organe approprié survivent en conservant leur métabolisme propre, en l'absence de tout processus de rejet. Dans ces conditions la persistance fonctionnelle des hépatocytes greffés pourrait être mise à profit dans le traitement des déficiences enzymatiques du foie.

Les applications cliniques possibles de ce type de transplantation suscitent encore beaucoup de questions, bien que les indications paraissent aujourd'hui plus définies. Initialement la transplantation d"hépatocytes a été proposée comme une méthode de traitement des déficiences enzymatiques et de l'insuffisance hépatique aigüe. Pour ce qui est de cette dernière indication, d'autres auteurs ont montré que la transplantation d'hépatocytes fonctionnels n'est pas nécessaire pour obtenir les résultats observés. Il a été prouvé qu'une substance encore inconnue, issue de ces cellules, stimule le rétablissement du foie malade du receveur. En effet, des extraits hépatiques apparaissaient être aussi effectifs dans le traitement de l"insuffisance hépatique aigüe. Par contre, il a été demontré dans cette thèse que le succès de la greffe d'hépatocytes dans le traitement des déficiences enzymatiques était fonction de toute la machinerie hépatocellulaire. Dès lors, l'indication potentielle de transplantation d'hépatocytes semble limitée actuellement au seul traitement des déficiences enzymatiques.

Il persiste néanmoins le problème de la quantité restrainte des cellules greffées nécessaire à l'obtention d'une guérison totale (comme dans les expériences avec les rats ictériques). Remarquons que la quantité de tissu de donneur, nécessaire pour la conjugation de toute la bilirubine, peut être beaucoup plus importante que la quantité de tissu hépatique nécessaire au traitement d'autres déficiences enzymatiques. En effet, dans un certain nombre de ces affections, la guérison clinique pourrait être obtenue par la présence d'une très petite quantité de l'enzyme déficient (par exemple dans la phénylcétonurie). Dans ce contexte, il est important de mentionner que la prolifération spontanée des hépatocytes greffés dans la rate conduit à une augmentation lente de la masse de tissu hépatique du donneur. En outre, il ressort de cette thèse qu'il est possible d'influencer l'activité de réplication de ces cellules.

La combinaison de la transplantation hépatocellulaire et de la technologie recombinante de $\mathrm{l}^{*} \mathrm{ADN}$ pourrait représenter un développement important et intéressant. En principe il est possible actuellement de recombiner le matériel génétique des hépatocytes isolés ou en culture (venant d'un patient avec une déficience enzymatique) avec la séquence de l'ADN nécessaire pour la fabrication de l'enzyme déficient. L'autotransplantation de ces hépatocytes "'manipulés' éliminerait le risque de rejet et pourrait conduire à la guérison du malade. Les données obtenues dans cette thèse constituent une base rationelle pour l'application d'un tel traitement. Bien que des études ultérieures sur cette forme de traitement s'avèrent nécessaires, il semble correct de conclure que la transplantation d'hépatocytes offre des perspectives de traitement des déficiences enzymatiques. 


\section{Samenvatting en conclusies}

Momenteel bestaat er voor de meeste erfelijke stofwisselingsziekten, die berusten op een enzymdeficiëntie, nog geen bevredigende behandelingswijze. Experimenteel zowel als klinisch is aangetoond dat de transplantatie van een lever van een gezonde donor de genezing kan bewerkstelligen van enzymdeficienties die hun oorsprong vinden in de lever. De indicatie voor een levertransplantatie in deze gevallen is echter de begeleidende of door de afwezigheid van het enzym veroorzaakte cirrhose, en niet primair de enzymdeficientie. Een aantrekkelijke benaderingswijze voor de behandeling van deze ziekten in een eerdere fase zou de transplantatie van levercellen kunnen zijn. Deze techniek zou belangrijke voordelen bieden ten opzichte van levertransplantatie. De operatieve ingreep draagt minder risico, de donorcellen kunnen d.m.v. bevriezing gepreserveerd worden, om op een gunstig moment gebruikt te worden. Bovendien zouden meerdere patienten behandeld kunnen worden met de levercellen verkregen uit een enkel donororgaan.

In dit proefschrift worden een aantal aspecten van experimentele leverceltransplantatie bestudeerd met betrekking tot de behandeling van erfelijke enzymdeficienties. Hoofdstuk 1 geeft een literatuuroverzicht over de tot dan bereikte resultaten met experimentele leverceltransplantatie, en bevat een inleiding tot de verrichte experimenten.

De eerste verhandelingen over leverceltransplantatie beschrijven hoe deze transplantatietechniek een genezende werking uitoefent op de Gunn rat, een rattenstam, die lijdt aan een overerfbare af wezigheid van het leverenzym bilirubine glucuronyltransferase. Dit enzym bindt galkleurstof (bilirubine) aan een suiker, waardoor dit afvalprodukt makkelijk via de gal wordt uitgescheiden. Door het totaal ontbreken van dit enzym bestaat er een levenslange geelzucht (icterus) bij de betreffende rat. $\mathrm{Na}$ de transplantatie van levercellen afkomstig van normale ratten bleek het bilirubinegehalte in het bloed van de Gunn rat te dalen. De wijze waarop leverceltransplantatie dit effect teweegbrengt, is onderwerp van studie in Hoofdstuk 2. Een duidelijke, maar slechts tijdelijke verlaging van het bloedgehalte aan bilirubine werd bereikt, wanneer in de milt van Gunn ratten levercellen van een gezonde rattenstam werden ingespoten (allogene transplantatie). Wanneer levercellen van Gunn ratten werden gebruikt (syngene transplantatie), werd geen bilirubinedaling waargenomen. Het bleek dat de normale levercellen werden afgestoten, en dat de Gunn levercellen werden geaccepteerd door de ontvangende Gunn rat en overleefden in de milt. Door de gal te bestuderen van bestraalde Gunn ratten (die door de bestraling tijdelijk niet konden afstoten) werd duidelijk dat bij de aanwezigheid van levende normale levercellen in de Gunn rat, de produkten van het ontbrekende enzym werden gevormd (het. "gebonden" 'bilirubine, oftewel de bilirubineglucuronides). Deze stoffen werden niet teruggevonden wanneer gedode normale levercellen of Gunn levercellen werden getransplanteerd. Wanneer er geen bestraling werd gegeven bleek, dat de gal van Gunn ratten die 
normale levercellen hadden ontvangen slechts gedurende korte tijd bilirubineglucuronides bevatte. Uit deze gegevens kon worden geconcludeerd dat de bilirubinedaling in de Gunn rat na leverceltransplantatie werd veroorzaakt door suppletie van het deficiente enzym. Deze enzymwerking vond zijn oorsprong in de getransplanteerde levercellen van de normale donorrat, en verdween wanneer deze donorcellen werden afgestoten.

Om de uitwerking van leverceltransplantatie in de icterische rat op lange termijn te kunnen bestuderen was het noodzakelijk ervoor te zorgen dat de levercellen van normale ratten niet werden afgestoten. Er werd in het Proefdierencentrum van de Katholieke Universiteit in Leuven, België, uitgaande van de Gunn rat en een normale rattenstam (de R/APfd stam), een nieuwe rattenstam gekweekt, die dezelfde enzymdeficientie heeft als de Gunn rat, maar verder de erfelijke eigenschappen bezit van de normale R/APfd rattenstam. Dit resulteerde in het ontstaan van de R/APfd-j/j stam $(j / j$ geeft de erfelijke enzymdeficientie aan). Omdat de overige erfelijke eigenschappen van de $R / A P f d-j / j$ rat nu gelijk zijn aan die van de $R / A P f d$ rat, worden cellen van de normale R/APfd rat niet als "vreemd" herkend, en dus niet afgestoten door de $\mathrm{R} / \mathrm{APfd}-\mathrm{j} / \mathrm{j}$ rat (congene stammen). De totstandkoming van deze nieuwe $\mathrm{R} / \mathrm{APfd}-\mathrm{j} / \mathrm{j}$ rattenstam en de proeven, die werden verricht om zowel de enzymdeficientie als de congeniciteit met de R/APfd stam aan te tonen, worden beschreven in Hoofdstuk 3.

In Hoofdstuk 4 wordt een studie beschreven met als vraagstelling of leverceltransplantatie ook een langdurige genezende werking heeft op een enzymdeficiente rat. In deze studie werd gebruik gemaakt van de congenetische R/APfd en R/APfd$\mathrm{j} / \mathrm{j}$ stammen. Een blijvende daling van het bilirubine gehalte trad op in de R/APfd$\mathrm{j} / \mathrm{j}$ rat, na injectie van R/APfd levercellen in de milt. Zes maanden na transplantatie bleek de gal van de getransplanteerde dieren nog steeds bilirubineglucuronides te bevatten, ten teken van de suppletie van het deficiente enzym. Tevens werd aangetoond met histologische technieken dat de levercellen van de gezonde donoren overleefden in de milt van de deficiente ratten. Na het verwijderen van de milt bleek dat er nog steeds (alhoewel veel minder) bilirubineglucuronides gevormd werden in de behandelde rat. Dit werd toegeschreven aan het doorstromen van donorcellen via de poortader naar de lever van de getransplanteerde ratten, alwaar deze cellen ook overleven. In het algemeen wordt de milt beschouwd als een goede plaats om levercellen in te transplanteren, omdat deze in dit orgaan kunnen overleven, en ook makkelijk bestudeerd kunnen worden met gangbare histologische technieken. Gezien het feit dat de alvleesklier (pancreas) van dezelfde embryologische oorsprong is als de lever en de bron is van hormonen die een werking uitoefenen op de lever, werd een onderzoek uitgevoerd om de pancreas te testen als ontvangend orgaan van de levercellen. Deze experimenten worden beschreven in Hoofdstuk 5. Levercellen afkomstig van ratten van eenzelfde ingeteelde stam bleken zich te nestelen in de pancreas en na 3 maanden nog steeds tekenen te tonen van normale stofwisselingsprocessen. Wanneer in de pancreas van $R / A P f d-j / j$ ratten levercellen van $R / A P f d$ ratten werden ingespoten, bleek dit te resulteren in verlaging van het bilirubinegehalte, en de vorming van bilirubine- 
glucuronides. Desondanks bleek in hetzelfde experiment dat leverceltransplantatie in de milt effectiever was dan transplantatie in de pancreas, gemeten aan de hoeveelheid gevormde bilirubineglucuronides.

In de voorafgaande experimenten werd duidelijk aangetoond dat leverceltransplantatie een therapeutisch effect had in de icterische rat. Een normalisering van het bilirubine gehalte werd echter nooit bereikt. De hoeveel heid getransplanteerde cellen was waarschijnlijk de beperkende faktor. In de experimenten van Hoofdstuk 4 werd er, na de transplantatie van $R$ /APfd levercellen in de milt van icterische $R / A P f d-j / j$ ratten, een aanvankelijk snelle en blijvende bilirubinedaling vastgesteld, gevolgd door een verdere langzame daling. Dit deed het vermoeden rijzen dat een delingsproces van de R/APfd levercellen een toename van de hoeveelheid niet-deficient leverweefsel veroorzaakte. Hiertegen pleitte echter dat bij routine histologisch onderzoek in onze experimenten nooit tekenen van zulk delingsproces waren waargenomen. Ook de literatuurgegevens waren vaak tegenstrijdig wat betreft het delingsgedrag van getransplanteerde levercellen. Deze kwestie werd nader bestudeerd in Hoofdstuk 6 . Hierbij werd gebruik gemaakt van een techniek, die cellen welke hun erfelijk (DNA) materiaal verdubbelen (een onderdeel van het celdelingsproces), herkenbaar maakt in histologische preparaten. Door middel van deze techniek kon worden aangetoond dat levercellen van donorratten van dezelfde soort als de ontvanger (syngene transplantatie) na transplantatie in de milt een continu delingsproces vertoonden. De studies werden verricht 12 en 20 weken na transplantatie. De proliferatie-index (dat gedeelte van de levercellen, dat in deling is op een bepaald moment) bedroeg ongeveer $3 \%$. Bovendien werd een methode ontwikkeld om de hoeveelheid leverweefsel in de milt te kunnen bepalen. De hoeveelheid van een bepaald enzym (GLDH), dat in grote concentraties in de lever, en in lage concentraties in de milt voorkomt, werd gemeten in de milt van getransplanteerde ratten. Op basis van deze metingen werd de hoeveelheid leverweefsel in de milt bepaald. Met behulp van deze methode kon worden aangetoond dat het continue delingsproces van donorcellen resulteerde in een toename van de hoeveelheid leverweefsel in de milt van het getransplanteerde dier. Wanneer de lever van de getransplanteerde rat gedeeltelijk werd verwijderd (dit geeft aanleiding tot snelle deling van levercellen ter compensatie van het verwijderde leverweefsel) bleek dat ook de getransplanteerde levercellen kortdurend werden gestimuleerd tot deling. Dit veroorzaakte echter geen extra toename van donor leverweefsel in vergelijking met de spontane groei ervan. De duur van de stimulatie tot deling was waarschijnlijk te kort om zo'n toename te bewerkstelligen.

In voorgaande experimenten werd de overleving van getransplanteerde levercellen geverifieerd door middel van histologisch onderzoek van de milt. Hiertoe moest echter het proefdier opgeofferd worden. In Hoofdstuk 7 wordt een nieuwe methode geevalueerd om de transplantaatoverleving te volgen: dynamische ${ }^{99 m}$ Tc-HIDA scintigrafie. ${ }^{99 \mathrm{n}} \mathrm{Tc}$-HIDA is een radioactieve st of die specifiek door levercellen wordt opgenomen en vervolgens wordt uitgescheiden via de galwegen. ${ }^{99 m} \mathrm{Tc}-\mathrm{HIDA}$ bleek opgenomen te worden in de milt van $\mathbb{R} / \mathrm{APfd}-\mathrm{j} / \mathrm{j}$ ratten, die 3 maanden tevoren een leverceltransplantatie (met R/APfd levercellen) in de milt hadden ondergaan. Dit gebeurde 
niet in de milt van R/APfd-j/j ratten die in plaats van een leverceloplossing een injectie van een zoutoplossing in de milt hadden ontvangen. Het histologisch onderzoek en de galanalyse op de aanwezigheid van bilirubineglucuronides bleken te correleren met dit scintigrafisch onderzoek. Uit deze studie bleek bovendien dat de snelheid van $9 \%$ Tc-HIDA opname en uitscheiding in de milt ongeveer dezelfde was als de opname- en uitscheidingssnelheid in de lever. Dit illustreerde dat stofwisselingsprocessen in de getransplanteerde levercellen normaal plaatsvonden. ${ }^{99 \mathrm{~m}} \mathrm{Tc}-$ HIDA scintigrafie heeft het voordeel dat herhaaldelijke metingen in eenzelfde proefdier mogelijk zijn, en is daarom een elegante methode om transplantaatoverleving aan te tonen.

Samenvattend kan gezegd worden dat de onderzoeken in dit proefschrift hebben aangetoond dat geïsoleerde levercellen na transplantatie in een geschikt orgaan overleven met behoud van hun specifieke stofwisseling, wanneer afstoting kan worden voorkomen. Het feit dat levercellen na transplantatie hun functie behouden kan worden toegepast in de behandeling van stofwisselingsziekten van de lever. Dit werd aangetoond in proeven met enzymdeficiente ratten.

Ten aanzien van de mogelijke klinische toepassingen van leverceltransplantatie blijven er vragen open. Nochtans lijken de toekomstmogelijkheden van leverceltransplantatie nu beter omlijnd. Aanvankelijk werd leverceltransplantatie een rol toebedacht bij de behandeling van enzymdeficienties en bij ernstige leverziektes. Deze laatste indicatie lijkt minder waarschijnlijk. Uit het werk van anderen bleek namelijk dat niet de transplantatie van levende cellen, maar een nog onbekende stof afkomstig van deze cellen, de zieke lever van de ontvanger sneller deed herstellen. Leverextracten bleken even effectief in de behandeling van deze leverziekten. In dit proefschrift werd experimenteel aangetoond, dat het succes van leverceltransplantatie bij enzymdeficienties wél afhangt van de aanwezigheid van overlevende nietdeficiente levercellen. Leverceltransplantatie lijkt daarom mogelijk een toepassing te hebben in de behandeling van enzymdeficienties.

Een probleem zou kunnen zijn dat de hoeveelheid getransplanteerde cellen te klein zou blijken om een volledige genezing te verkrijgen (zoals dit inderdaad het geval was in de onderhavige proeven met icterische ratten). Hierbij moet opgemerkt worden, dat de hoeveelheid donorweefsel benodigd voor de conjugatie van al het gevormde bilirubine, veel groter kan zijn dan de hoeveelheid leverweefsel die nodig is voor de behandeling van andere enzymdeficienties. In een aantal van deze ziekten zou de aanwezigheid van slechts een zeer geringe hoeveelheid van het ontbrekende enzym al genoeg zijn om een klinische genezing te kunnen bewerkstelligen. In deze context is het ook vermeldenswaard dat de levercellen na transplantatie in de milt een spontaan celdelingsproces vertonen, hetgeen een langzame toename veroorzaakt van de hoeveelheid donorweefsel. Bovendien is uit dit proefschrift gebleken dat het mogelijk is de delingsactiviteit van deze cellen positief te beinvloeden.

De combinatie van leverceltransplantatie en DNA technologie zou een belangrijke en boeiende ontwikkeling zijn. In beginsel is het tegenwoordig mogelijk in geisoleer- 
de levercellen afkomstig van een patient met een enzymdeficientie, het benodigde genetische materiaal in te brengen om de produktie van het ontbrekende enzym mogelijk te maken (DNA recombinatie technologie). De transplantatie van deze "bewerkte" lichaamseigen levercellen zou zonder afstotingsproblemen zijn en kunnen resulteren in de genezing van de patient. De gegevens die in dit proefschrift werden gepresenteerd vormen een rationele basis voor het uitvoeren van zo" $n$ behandeling. 


\section{Acknowledgements}

During the five years of work on the realization of this thesis, I have not only enjoyed an enormous amount of substantial help, but also of moral support and friendship from many people, for which I am very grateful.

First of all, I would like to thank those people, without whom this thesis would not have seen the light, and to whom this work is dedicated.

Dear Christiane, it must have been love (and a fair bit of your French temperament) that gave you the strength to cope with your often "impossible" husband during the activities in the laboratory. You have helped me to complete this thesis in every way imaginable. Merci pour tout, chérie!

Dear father and mother, you provided a great, solid starting-base into life as well as a comforting home-port in later times. I owe you my deepest respect and gratitude.

Prof. G. Kootstra, dear Gauke, your lively interest, tactful guidance and continuous support have always stimulated me to complete this work. Especially your characteristical optimism has helped me tremendously, when setbacks in the experimental work disconcerted me.

Dr. W. Buurman, dear Wim, thank you for all the time and energy spent to realize this thesis. You were a real fine tutor. (Is this short enough?).

Dr. C. van der Linden, dear Cees, I will always remember with pleasure the evenings of our combined sporting and scientific activities. Let us try to keep up a good balance between these two matters!

Prof. C. Greep, your dynamism as an organizer in health affairs has almost become proverbial. Thanks to the infrastructure you have created at our department and university I was able to finish my experimental work during my surgical residency.

Special thanks go to the research group of the Laboratory of Hepatology at the Katholieke Universiteit Leuven. Prof. K. Heirwegh was always willing to comment on the design of the studies, and critically reviewed the final manuscript. His and Dr. N. Blanckaert's expertise on bilirubin metabolism have enhanced the quality of this work. Most bile analyses were performed in their laboratory.

Prof. J. Flendrig, Prof. D. Meyer and Prof. R. Pichlmayr carefully reviewed the final manuscript of this thesis.

I acknowledge gratefully the cooperation of the Laboratory Animal Center (Proefdierencentrum) in Leuven and the Centralized Experimental Animal Facilities (Centrale Proefdier Voorzieningen) in Maastricht. The directors, Ir. R. Leyten and Dr. A. van den Bogaard, respectively, and the personnel of these institutions were always of great help, and their assistance was greatly appreciated.

The scintigraphic studies were conducted in the Laboratory of Nuclear Medicine un- 
der supervision of Dr. J. Coenegracht, whose contribution to Chapter 7 was extremely valuable. $\mathrm{Dr}$. Th. Postmes assisted at the very beginning of my scientific endeavours, and has continued to show his interest in my work.

Furthermore I am very much endebted to I. Daemen, C. Denolf, J. van Dongen, B. Engelen, T. Jeunhomme, Dr. J. Maessen, G. Michiels, R. Michiels, G. Pauwell, R. Remy, M. Reijnders, N. Rijskamp, H. Simons, Dr. B. Schutte, J. Stessel, P. Willems, Dr. R. Visser and C. Voskamp, who all contributed to a specific part of the thesis, and whose names are also mentioned either in the authors lists or in the acknowledgements of the chapters involved.

J. Houben and A. Giebels of the Audiovisual Department never gave up trying to improve the quality of the figures.

Dr. M. Meurice from Liège reviewed and corrected the French summary. Although often mentioned in the acknowledgements of the preceding chapters I would like to express my sincere thanks once again to Mrs. K. Spronck for her secretarial assistance.

Finally, I feel much obliged to all my colleagues of the Department of Surgery for their good-fellowship during the years of my residency. 


\section{Curriculum vitae}

The author of this thesis was born in Gelleen on February 24, 1954.

After finishing high school (Gymnasium $\beta$ ) in 1972, he went to medical school at the Catholic University of Leuven (Belgium). In 1979 he graduated and served her Majesty's army as a Medical Officer till 1980. In 1980 he was employed at the Department of Surgery (Chairman: Prof. Dr. J.M. Greep) of the Academic Hospital Maastricht as a surgical resident. His surgical training was interrupted from July 1981 till November 1982, when he functioned as a transplant coordinator at the same department. Registration as a general surgeon is due in the course of 1987. 

Druk: Groenevelt bv, Landgraaf 